\title{
WestVirginiaUniversity
}

THE RESEARCH REPOSITORY @ WVU

Graduate Theses, Dissertations, and Problem Reports

2005

\section{Integrated stability mapping system for mines}

Quanxi Wang

West Virginia University

Follow this and additional works at: https://researchrepository.wvu.edu/etd

\section{Recommended Citation}

Wang, Quanxi, "Integrated stability mapping system for mines" (2005). Graduate Theses, Dissertations, and Problem Reports. 4203.

https://researchrepository.wvu.edu/etd/4203

This Dissertation is protected by copyright and/or related rights. It has been brought to you by the The Research Repository @ WVU with permission from the rights-holder(s). You are free to use this Dissertation in any way that is permitted by the copyright and related rights legislation that applies to your use. For other uses you must obtain permission from the rights-holder(s) directly, unless additional rights are indicated by a Creative Commons license in the record and/ or on the work itself. This Dissertation has been accepted for inclusion in WVU Graduate Theses, Dissertations, and Problem Reports collection by an authorized administrator of The Research Repository @ WVU.

For more information, please contact researchrepository@mail.wvu.edu. 


\title{
Integrated Stability Mapping System for Mines
}

\author{
Quanxi Wang \\ Dissertation submitted to the \\ College of Engineering and Mineral Resources \\ at West Virginia University \\ in partial fulfillment of the requirements \\ for the degree of \\ Doctor of Philosophy \\ in \\ Mining Engineering
}

\author{
Keith A. Heasley, Ph. D., Chair \\ Syd S. Peng, Ph. D. \\ Yi Luo, Ph. D. \\ James D. Mooney, Ph. D. \\ Christopher Mark, Ph. D. \\ Department of Mining Engineering \\ West Virginia University \\ 2005
}

Keywords: Hazard mapping, Geologic factor, Structure features Stress analysis, Stability index 


\section{ABSTRACT \\ Integrated Stability Mapping System for Mines}

\section{Quanxi Wang}

The Integrated Stability Mapping System (ISMS) was developed as an engineering tool to quantify the geologic and geo-mechanical information of mines, and to integrate the critical stability influence factors into an overall stability index for use in mine planning and support design. It is generally understood that the inherent underground roof stability is determined by the interaction of both the given geologic characteristics and the local stress influences. Form this perspective, in this dissertation, the need for an integrated stability mapping system is established through investigating the traditional and current hazard mapping practices. In order to fulfill this need, computer aided hazard mapping techniques and popular numerical methods for geomechanical analysis are reviewed. Then, an integrated stability mapping system incorporating geology hazard mapping, geologic structural feature impacts, and advanced numerical stress analysis techniques into one solution has been developed.

The stability system is implemented inside the de-facto standard drawing environment, AutoCAD, and in compatible with widely used geology modeling software SurvCADD. This feature allows one to access numerous existing geologic data and mining information from present mine maps easily and directly. The LaModel stress calculation, a boundary element method, integrated within the mapping system can produce realistic and accurate stress and displacement analysis with its distinguished features such as the laminated overburden model, the true topography consideration and actual irregular pillar matching.

After the stability mapping system was developed, two case studies were performed to check for coding errors, calculation accuracy, and for demonstrating the functionalities and usefulness of the system. In the case studies, the composite stability index was compared with field observations. A good correlation has been found although only a few influence factors have been considered.

In the conclusion of this dissertation, it is suggested that the stability mapping system provides mining engineers with the ability to perform comprehensive, rapid and accurate multiple-factor stability mapping analysis. Then the resultant stability map can be a valuable guide to safer support designing and better mine planning, and ultimately increase the safety of mine design and reduce the injuries and fatalities associated with ground fall in underground mines. 


\section{ACKNOWLEDGEMENTS}

Completion of this dissertation would have been impossible without the guidance and help from my advisors, colleagues, and friends and family. I would like to express sincere appreciations to following individuals:

Foremost, my thanks have to go to Dr. Keith A. Heasley, my advisor, for his support, encouragement and advice during both my research and course work in WVU. His experiences in computer application in numerical modeling, mining design and rock mechanics have constantly inspired me.

I am grateful to Dr. Syd S. Peng, Dr. Yi Luo, Dr. James Mooney, and Dr. Chris Mark for their time serving on the committee and for providing important perspectives to the research work of this dissertation.

This work was performed under a project sponsored by Notional Institute for Occupational Safety and Health (NIOSH). Special thanks go to Dr. Chris Mark, Section Chief of Rock Mechanics, NIOSH, for the financial support.

In particular, I am indebted to my sister and parents in law for their support and encouragement.

More importantly, I want to thank my wife, Chunmei and our daughter, Annie for their love, patience, support and encouragement during my study in Morganton. 


\section{TABLE OF CONTENTS}

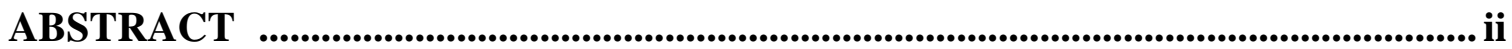

ACKNOWLEDGEMENTS ......................................................................................... ii

TABLE OF FIGURES....................................................................................... vii

Chapter 1. Introduction......................................................................................1

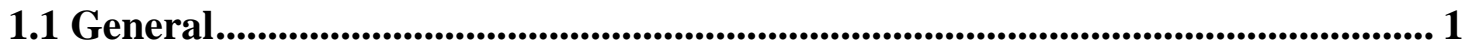

1.2 Statement of the Problem................................................................................... 3

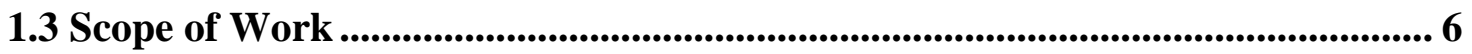

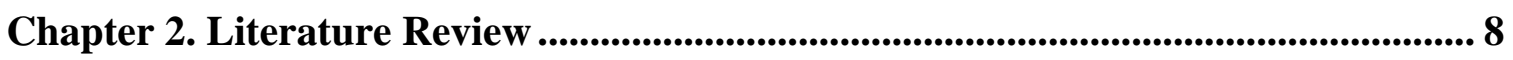

2.1 Traditional Geologic Stability Mapping ................................................................. 8

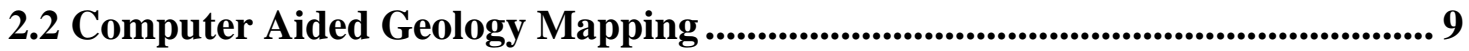

2.3 The Influence Factors on Mine Stability ......................................................... 12

2.4 Stability Mapping with Multiple Influence Factors .......................................... 14

2.5 Numerical Methods on Mine Stability Analysis......................................... 23

2.5.1 Numerical Methods.................................................................................. 23

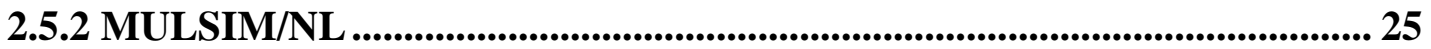

2.5.3 LaModel and its Recent Development ................................................... 28

2.6 AutoCAD Customization and Programming .................................................. 32

2.6.1 AutoLISP language .......................................................................................... 33

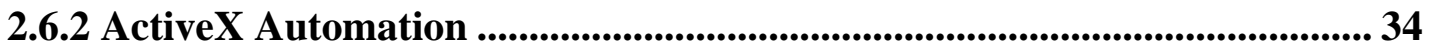

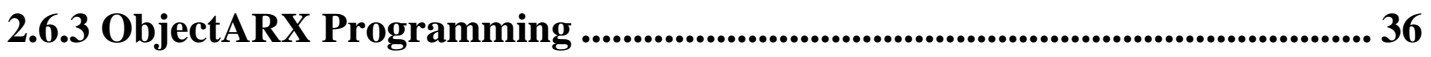

Chapter 3. Design of Integrated Stability Mapping System ........................................ 39 


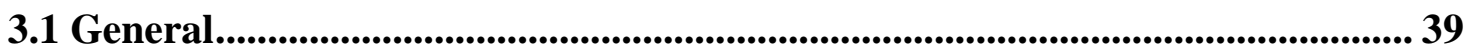

3.2 Structure of Integrated Stability System .................................................................... 39

3.3 Requirements for Input Data.................................................................................... 43

3.4 Stability Mapping Modules................................................................................................ 44

3.4.1 Geology and Geo-Structural Gridding Modules.............................................. 45

3.4.2 Stress Analysis Modules ........................................................................................ 46

3.4.3 Horizontal Stress Module.......................................................................................... 47

3.4.4 Stability Factor Transformation ........................................................................... 48

3.4.5 Stability Index Modules...................................................................................... 49

3.4.6 Grid Utility Module ................................................................................................ 50

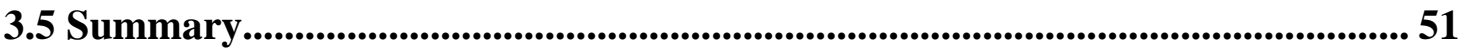

Chapter 4. Implementation of the Stability Mapping System ........................................ 52

4.1 General.................................................................................................................... 52

4.2 Fundamental Data Structure of the System .......................................................... 52

4.3 Geology Oriented Modules .................................................................................... 53

4.3.1 Gridding Module for Spatial Points ................................................................... 55

4.3.2 Gridding Module for Contours............................................................................... 58

4.3.3 Gridding Module for Linear Features ............................................................... 60

4.3.4 Gridding Module for Area-Based Features....................................................... 62

4.4 Stress Oriented Modules .......................................................................................... 67

4.4.1 Overburden Stress Module ....................................................................................... 68

4.4.2 Horizontal Stress Module........................................................................................ 71

4.5 Index Mapping Modules ....................................................................................... 73 
4.5.1 Stability Factor Transformation ......................................................................... 73

4.5.2 Final Stability Index Module ...................................................................... 81

4.5.3 Final Index Mapping Module ................................................................ 83

4.6 Summary

Chapter 5. Interfaces and Constraints .............................................................. 86

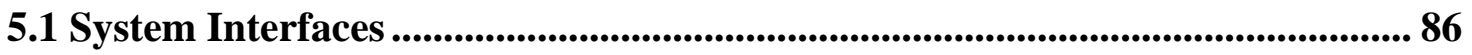

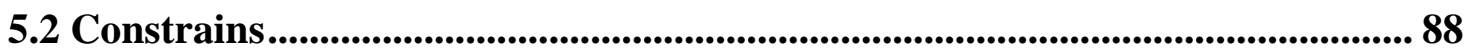

Chapter 6. Case Studies and System Validation ............................................................. 90

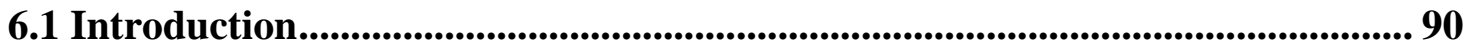

6.2 The Case study one ............................................................................................. 90

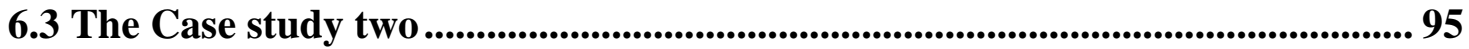

6.3.1 Background Information................................................................... 95

6.3.2 Stability Mapping Process................................................................................ 98

6.3.3 Index Mapping and Field Verification................................................... 103

6.4 Application guidance ................................................................................... 106

Chapter 7. Summary and Conclusions ..................................................................... 108

7.1 Summary ........................................................................................................ 108

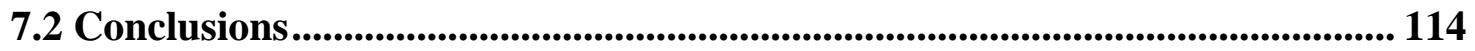

7.3 Ideas for Additional Research ...................................................................... 115

REFERENCES...................................................................................................... 118

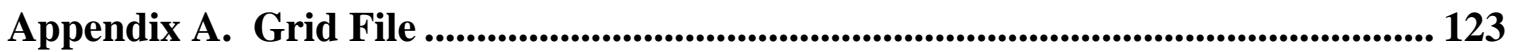

Appendix B. Menu System and Principal Input Forms ......................................... 125

Appendix C. AutoCAD Database Overview............................................................ 143 


\section{TABLE OF FIGURES}

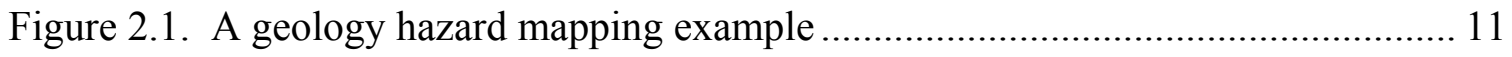

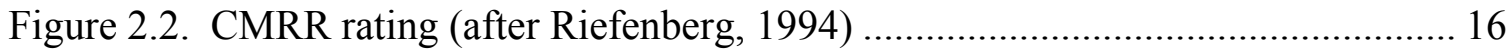

Figure 2.3. Vertical stress for upper and lower seam (after RiefenBerg, 1994) ............. 17

Figure 2.4. Individual factors and hazard map (after Riefenberg, 1994)...................... 18

Figure 2.5. Sandstone influence over a longwall panel (after Stankus et al., 2001)....... 19

Figure 2.6. Areas with and without stream valley influence (after Stankus et al., 2001) 20

Figure 2.7. Tectonic stress influence (after Stankus et al., 2001) ................................. 21

Figure 2.8. Multi-factor stability rating (after Stankus et al., 2001)............................. 21

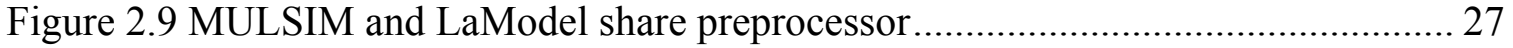

Figure 2.10. Laminated overburden model .................................................................. 29

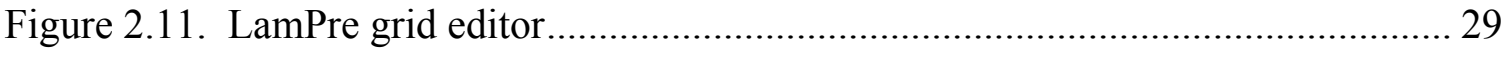

Figure 2.12. A typical LaModel vertical stress output............................................... 30

Figure 2.13. A close-up view of automatically generated mine grid in AutoCAD ......... 32

Figure 2.14. ObjectARX shares same address space with AutoCAD .......................... 37

Figure 3.1. Schematic structure of the integrated stability mapping system ................. 40

Figure 3.2. Typical data flow chart of stability mapping analysis ............................... 42

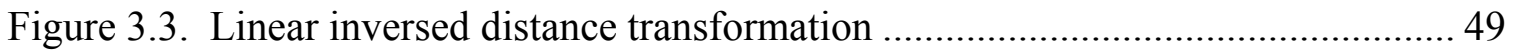

Figure 4.1 Details of the spatial point module ......................................................... 57

Figure 4.2 Details of the contour gridding module................................................ 59

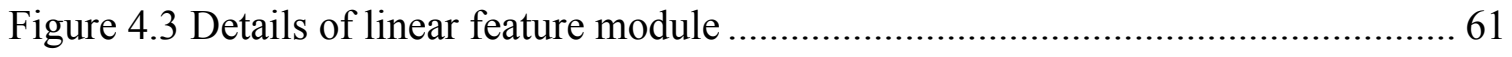

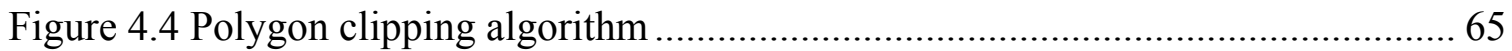


Figure 4.5 Details of gridding module for area-based feature .................................... 66

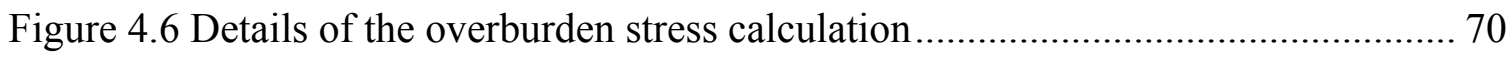

Figure 4.7 Details of the horizontal stress module ................................................ 72

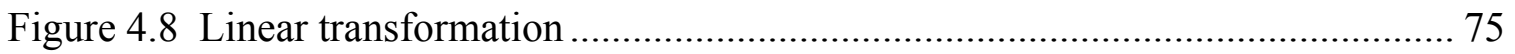

Figure 4.9 Exponential transformation ................................................................ 78

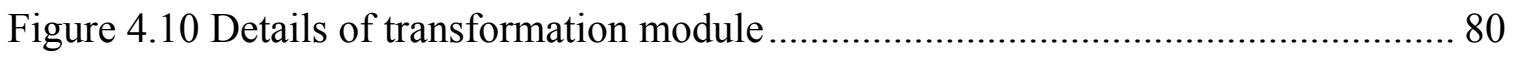

Figure 4.11 Details of final stability index calculation.......................................... 82

Figure 4.12 Details of stability index mapping module........................................... 84

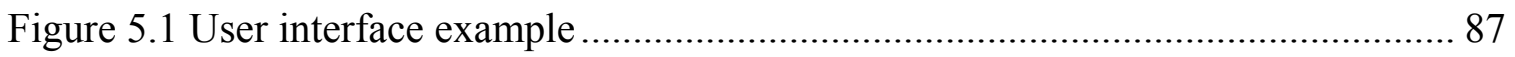

Figure 6.1 Map of the first case study area ........................................................ 91

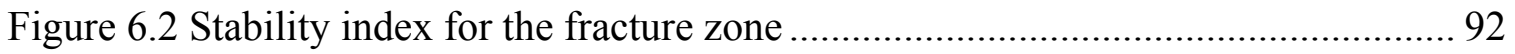

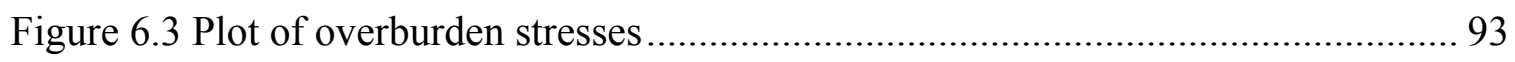

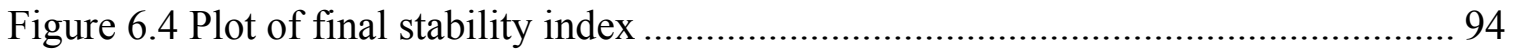

Figure 6.5 Comparison of observed roof condition with stability index ....................... 94

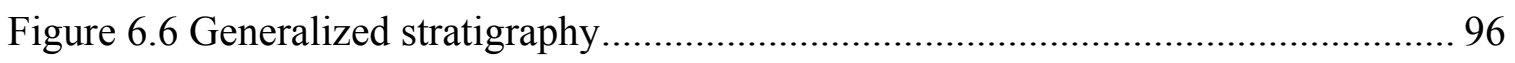

Figure 6.7 Plan view with mine layout, overburden, and the location of the case study. 97

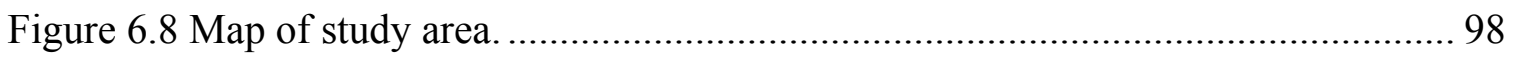

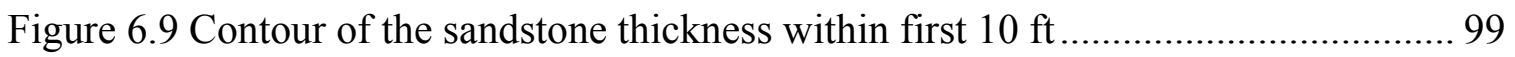

Figure 6.10 Sandstone percentage in the first $10 \mathrm{ft}$ roof....................................... 99

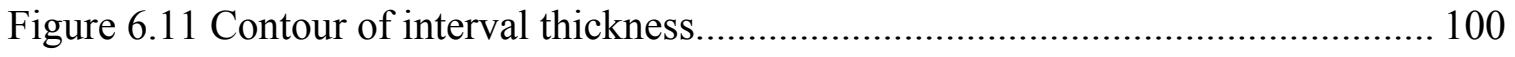

Figure 6.12 Thickness of the interval from seam to sandstone $(\mathrm{ft})$........................... 100

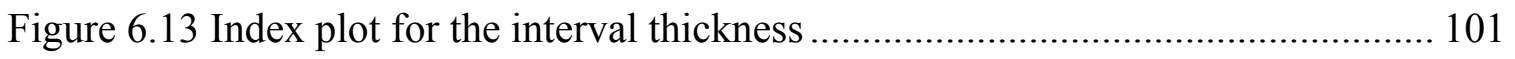

Figure 6.14 Index plot with an inverse step transformation ..................................... 102 


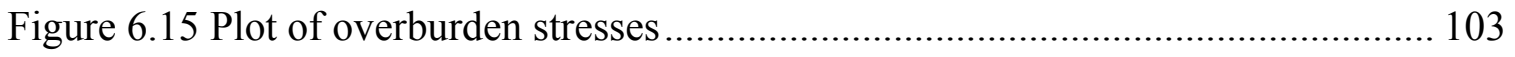

Figure 6.16 Stability index with equal weights .......................................................... 104

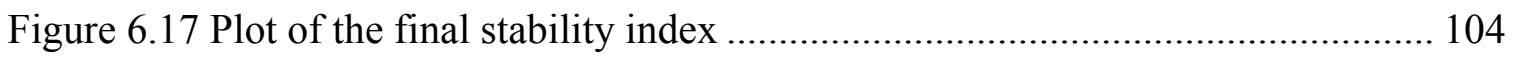

Figure 6.18 Comparison of observed pillar/rib condition with stability index.............. 105

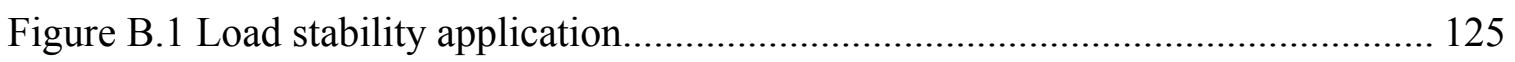

Figure B.2 Stability mapping menu system........................................................ 126

Figure B.3 Grid definition form....................................................................... 128

Figure B.4 Form of contour gridding module......................................................... 129

Figure B.5 Form for point gridding module ……………...................................... 130

Figure B.6 Form for linear geological feature module ................................................ 131

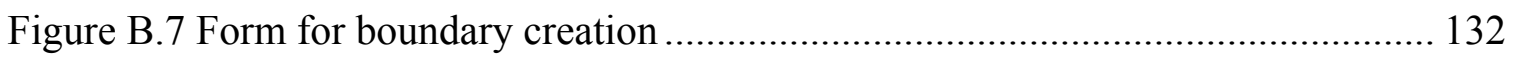

Figure B.8 Form for area based factors ................................................................. 133

Figure B.9 Form for surface grid in overburden stress module ................................... 134

Figure B.10 Form for seam grid in overburden stress module ..................................... 135

Figure B.11 Form for properties and control parameters ............................................ 136

Figure B.12 Form for horizontal stress influence ........................................................ 137

Figure B.13 Form for original grid in transformation module....................................... 138

Figure B.14 Form for influence factor transformation ................................................ 139

Figure B.15 Form for stability index generation …………..................................... 140

Figure B.16 Form for stability index mapping .......................................................... 141

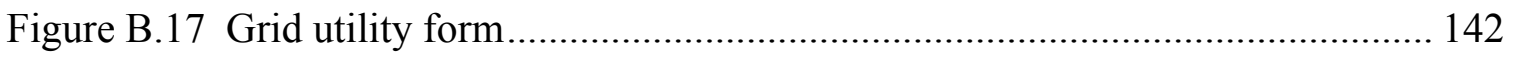




\section{Chapter 1. Introduction}

\subsection{General}

If a mining engineer wants to optimize the stability of a mine opening, there are three major factors that have to be considered: the geology (that nature has provided), the stress conditions (a combination of natural conditions, such as overburden and tectonics, and man-made conditions, such as pillar design, multiple-seam stresses, etc.) and the roof support (completely controlled by the engineer). Among these conditions, the geologic properties of the reserves undoubtedly play the most important role. In the past, mine design engineers have used the specific geologies of mines to develop geologic "hazard" maps, which show areas of the mine plan where poor or weak geology is expected (Stankus et al., 2001; Newman et al., 2001a, Riefenberg, 1994). With this foreknowledge, the miners could be prepared with modifications in the pillar design or roof support plan appropriate to compensate for the reduced geologic stability in that area.

However, it is generally understood that the inherent underground roof stability is often determined by the interaction of both the given geologic characteristics such as: rock strength, layer thickness, discontinuities spacing, etc. and the local earth mechanical influences such as: overburden stress, abutment stresses, multi-seams stresses, stream valley effects, etc. From this perspective, recently mining engineers and researchers have been trying to map both the geologic and geo-mechanic analysis data together in order to improve the understanding of the roof stability. 
Unfortunately, due to the lack of a universally applicable software and the diversity of available geology and geo-mechanical data, the information collecting, influence factor analyzing and stress modeling in past practice has been time consuming and arduous. Many tedious tasks have to be done either manually or separately by using different software packages from different sources. Given the complexity of the typical geologic and stress environment, it is very difficult for all of those many factors to be considered accurately and quantitatively when looking at the stability of the mine roof.

As a response to the historical difficulty and complexity of combined stability mapping, an Integrated Stability Mapping System (ISMS) has been developed to allow the mining engineer to effectively combine the geologic characteristics and stress influences into an integrated system for use in support design and mine planning. This stability mapping system utilizes the popular AutoCAD (with or without SurvCADD) platform as a foundation for automatically gathering and inputting the geologic characteristics, and then tightly integrates the boundary element program LaModel for determining the stress influences. The integrated stability program allows the user to apply various weighting functions to the input geology and stress factors, and then generates an overall stability index map for the mine property. This dissertation discusses the design principals of the integrated stability mapping system and presents its application through case studies. 


\subsection{Statement of the Problem}

A comprehensive stability analysis for an underground mine often requires considering multiple input parameters: geological properties and geo-mechanical stresses. The structural and geologic input can include such local characteristics as: rock strength, layer thickness, faults, stream valley effects, etc. The geo-mechanical stress inputs can include overburden stress, multiple-seam stresses, abutment stresses, etc. Performing such an analysis with the use of computer programs has become a common practice in underground mine design today.

For instance, to analyze the geology and produce a geologic map at a mining site, there are numerous software packages available. The most common package in coal mining (used at $80 \%$ of the U.S. coal mines, (Richards, 2001)) is "SurvCADD" by Carlson Software. SurvCADD is designed as an add-on to the AutoCAD drafting program. The SurvCADD package consists of multiple separate modules which assist the user with surveying, reserve estimation, mine planning and other tasks. Among many other capabilities, this program can quickly and easily map (contour or grid) geologic characteristics such as layer thickness, strength, chemical composition, etc. from a variety of input sources such as surface drill holes, underground samples or observations. These "geology" maps form the basis for developing a geologic stability map, which plots a geologic "stability factor" layered onto the mine plan

In the same time frame that some mining engineers were creating geologic hazard maps, other mining researchers were developing and applying stress programs in order to analyze the complex interaction of underground mine configurations. For example, a couple of these programs, MULSIM/NL and LaModel, were developed by the US Bureau 
of Mines (and then NIOSH) to analyze the stress and displacements associated with complex geometries and multiple-seam mining configurations (Heasley, 1998; Zipf 1992a, 1992b; Kripakov et al., 1988). These programs are based on the displacementdiscontinuity variation of the boundary-element method and are designed for modeling stress and displacements in thin, tabular seams with single or multiple-seam configurations.

In the past, mining engineers and researchers have used various software tools to develop stability map with multiple influence factors for specific mines (see section 2.4). However, there were many problems and limitations encountered during these practices:

Initially, these studies were conducted individually on specific sites and considered only limited stability factors. Due to the diversity of the geologic condition at each mine, a method used in one mine is hardly applicable to another mine.

Second, the data collecting and analysis are always time-consuming and arduous tasks, because there is no proper software available to facilitate the purpose, and very different packages have to be used separately and combined in the process. Meanwhile incorporating all the outputs with very different formats and interpretations from these individual programs is also not a trivial task.

In addition, the raw data used in stability mappings are usually extracted from various sources with varying formats and representations, which are not well prepared for automatic computer analysis. There often exist some types of problems such as impurities, misrepresentations, numeric and typographic errors. These problems will hinder or crash any automatic program process. As a result, a lot of data preparation has 
to be done manually and in advance. All of these conditions make it impossible to conduct rapid and accurate stability analysis and mapping.

Finally, in these studies, very different numerical stress analysis packages had been used such as the Finite Element Method, MULSIM/NL, etc. The Finite Element Method (FEM) is definitely a powerful technique widely used in industries. But in comparison to other widely used methods, the Finite Element Method is more computational intensive and has much higher resource demands. This generally means that the Finite Element Method is not suitable for the seam level analysis of large planar area. Also, applying FEM technique is quite problematic for practicing engineer because most available FEM packages are too complicated and involved for them to master in a busy schedule.

In summary, the current geologic mapping and geo-mechanical analysis systems were not specifically integrated because of the unique nature of the respective systems at that time. Often, the overlap or combined stability influence from both the geology and the stress may have been overlooked. Also, due to the specialized nature of the programs, the majority of the geologic hazard mapping and geo-mechanical stress analysis was performed by experts in those particular programs and areas. The practicing mining engineer could not find the time to learn and master these additional, or advanced, mine design programs. With the level of computer technology and sophistication in computer-aided geologic mapping and geo-mechanical stress modeling programs today, it is certainly feasible, and desirable, to combine these separate analyses into an integrated stability mapping approach. And with the new breed of mining engineers coming out of higher education with a solid background in both computer mapping and 
numerical analysis, it may be expected that the practicing mine engineer will be capable of successfully applying such an integrated program once it is developed.

\subsection{Scope of Work}

The objective of this research project is to design and develop an integrated stability mapping system, which is able to automatically gather and retrieve the geologic and geo-structural characteristics from various resources, and combine them with geomechanical stress analysis technologies to produce a site specific stability mapping index. Various transformation functions will be constructed to apply to the individual stability influence factors and an overall stability index can be generated by using the included weighting procedure.

The primary outcome from this research work is a computer program which produces a stability map showing an entry stability index layered onto the mine plan. This program output is intended to supply the practicing mine design engineer with additional knowledge to be used for designing more stable and safer mines. What is unique about the proposed program to be developed in this research is that it will integrate both the geology and the geo-mechanical stress in determining the stability index. Also, this will be the first program specifically developed from the beginning for determining a mine wide stability factor. Therefore, by design, it will incorporate numerous features to simplify and accelerate the input, computation and output of the stability index.

In developing a program which integrates geology and stress analysis for determining a stability index, a lot of previous work can, and should, certainly be incorporated in the development process. Numerous drafting and mine mapping packages exist in the 
industry upon which to build. Similarly, numerous geo-statistical and geologic mapping programs are available, and several different stress analysis programs might be used. Logically, the most appropriate and/or most widely-used program in each category would be the best choice for developing a program for maximum distribution and acceptance in the mining industry. As a result, AutoCAD/SurvCADD drafting software is chosen as a platform for the stability system due to its flexible customization capability and its popularity in mining industry, while LaModel stress calculation will be integrated into the system because of its outstanding features and wide acceptance by practicing mining engineers.

In order to make the system full-featured, the intention is to integrate a wide range of current geology and stress models into one package with a number of independent functional modules. The implementation will be based on commonly used data resources and algorithms with minimal input parameters to benefit the practical application of the system. In addition, a number of case studies will be performed which highlight the unique capabilities of the system and verify the functionalities by comparing the results with the actual field observations. 


\section{Chapter 2. Literature Review}

Stability mapping, also often called hazard mapping, is a common practice in mines to evaluate the roof stability of underground openings during and ahead of mining operations. It has been used in the mine industry for more than a century (Jiang and Wells, 1998; Riefenberger, 1994). Over time, with the increasing requirements for safety and the change of mining conditions from simple geology, shallow, single seam deposits to complex, deep, multi-seams situation, the progress of stability mapping also has evolved from straightforward geology modeling into a complicated multi-factor analysis.

\subsection{Traditional Geologic Stability Mapping}

Effective mine design has long been recognized as an essential element in establishing safe and productive mining operations (Karabin, 1994). In order to analyze the stability of underground openings and maximize the mining efficiency, one of the fundamental assignments of mining engineers is often to develop a stability map over the prospective area of the reserves. Typically, the graphical elements in the stability map would indicate and clarify the relative stability of the overall field situation. This information can be utilized to identify the potential unstable sections and guide the support design and mine planning.

In practice, the geologic mapping involves numerous tedious, time-consuming and multi-steps tasks. To produce a geologic stability map, the mine engineers or geologists have to collect the available geological data from depositional modeling, drill cores, and/or underground observations. These kinds of information may include the geology properties near the seam deposit, for example, seam and immediate roof thickness, 
strength, chemical contents of coal seam etc. Also the thickness of overburden and interburden of seams etc. may be collected. Other topography structures on the surface, such as stream valleys, rivers, highways and rail roads as well as the other abnormal geologic structures underground, for instance, faults and sandstone channels, are also noteworthy factors for the investigators. For the operating mine, the history of pillar stability and roof conditions could be recorded as good as possible by mapping the locations of roof falls, cuter roof, broken roof etc. This geologic mapping was historical done by hand successfully across the entire reserve during mine life. By examining stability elements on the map and combining with their experiences at a particular mine, an experienced mining engineer could have some confidences in identifying the stability distribution and could take the necessary precautions in the areas with higher probabilities of potential failure.

\subsection{Computer Aided Geology Mapping}

With the advance of computer technology, the original paper-based geology mine map has gradually transformed into an electronic map. The information on geology and production is stored or digitized into computer files. This can be accomplished by using a common computer aided design (CAD) software system from Autodesk ${ }^{\circledR}$, MicroStation ${ }^{\circledR}$, Mincom $^{\circledR}$ or other corporations. Among these different systems, the AutoCAD ${ }^{\circledR}$ drawing package from Autodesk has gained the greatest popularity in the US coal mining industry with some $90 \%$ of mines using the program for their mapping requirements (Heasley, 2001). Although AutoCAD is a general purpose computer-aided drafting application program designed for use on single-user, desktop personal computers and graphic 
workstations, it has been an essential tool in many industries, including mining, mechanical and civil engineering and architecture.

one of the most successful applications in the mining community is SurvCADD ${ }^{\circledR}$, developed by Carlson Software ${ }^{\circledR}$, which is used for surveying, mine planning and reserve calculation maps at about $75 \%--80 \%$ of the U. S. coal mines (Richards, 2001). SurvCADD is designed as an add-on application entirely within the AutoCAD environment. It consists of multiple separate mining modules which assist the user with surveying, reserve estimation, mine planning and other tasks. Among its many capabilities, this program can quickly and easily map (contour or grid) geologic characteristics such as layer thickness, strength, chemical composition, etc. from a variety of input sources such as surface drill holes, underground samples or observations (Carlson Software, 2000). Figure 2.1 is a typical example of a general geology hazard map where the various roof anomalous observed underground were carefully added to the electronic geology map with various distinguishing symbols for different type of roof problems. When the longwall panel passes through the area near where previous failures have occurred such as the middle of lower entry, the additional precautions may be taken to avoid possible roof falls in the adjacent entries. 


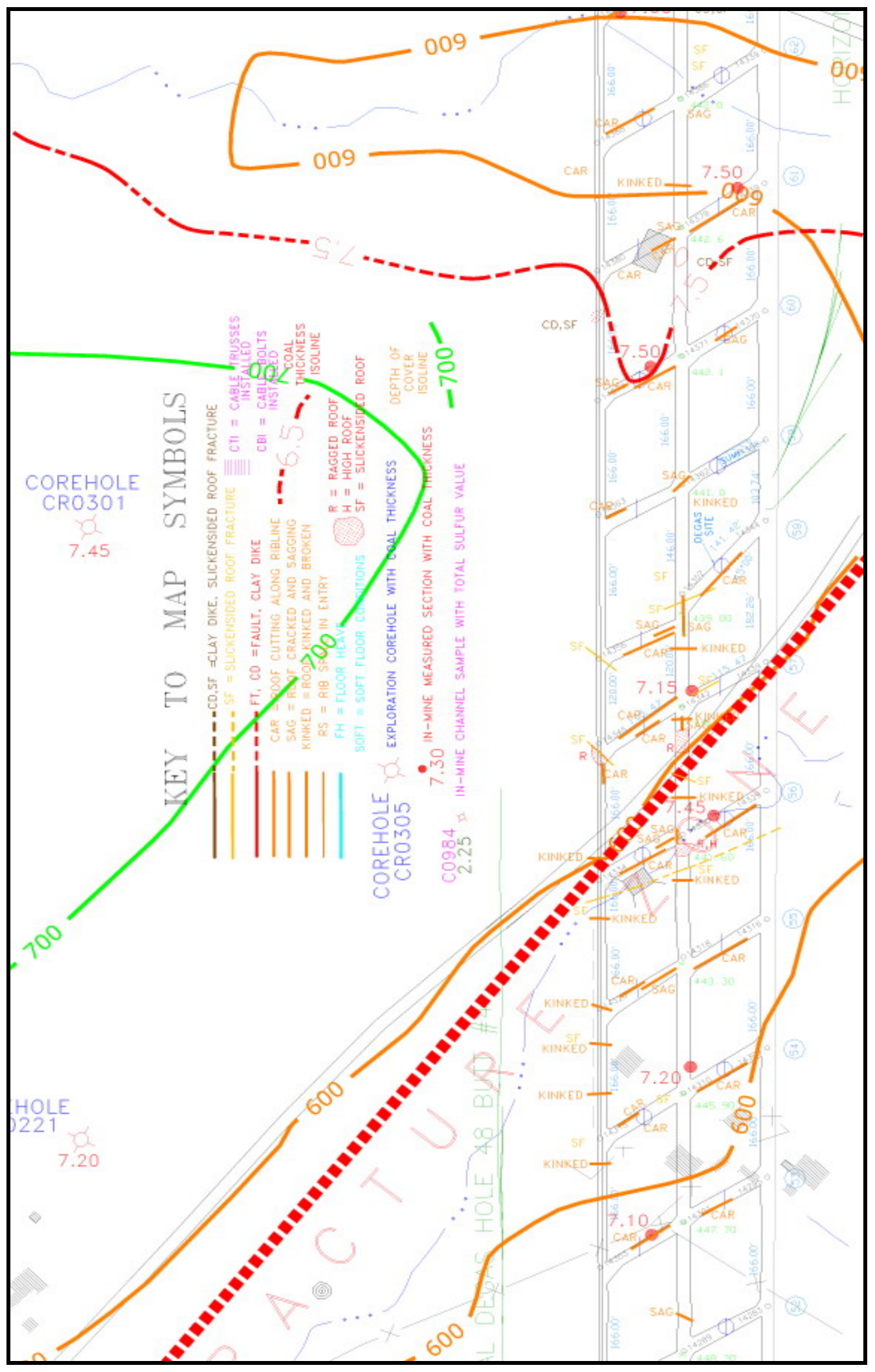

Figure 2.1. A geology hazard mapping example 


\subsection{The Influence Factors on Mine Stability}

The geologic properties of the surrounding strata are a fundamental factor for the determination of anticipated roof stability. These properties may include every aspect of the reserve deposition such as layer thickness, intact rock strength, presence of weak partings, chemical composition, etc. For example, in the Pittsburgh seam, sandstone channels present significant influences on roof stability. In the transition areas where sandstone exists, roof problems such as extensive slickensides and falls are often encountered (Stankus, 2001). Therefore, the thickness of the sandstone and the location of the sandstone layer above the coal seam are some of the critical factors for mines mining this seam. Typically, these wide-spread geologic factors can be determined from borehole data, underground mine opening observations and standard roof and rib bolting operations. For those geologic factors identified with the highest influence on the mine stability, a detailed information collection and investigation process can provided more reliable evidence for the stability mapping.

However, it is well known that a majority of mine roof problems results from some kinds of structurally anomalous conditions ( $\mathrm{Su}, 1999)$. Such anomalies are usually related to sudden changes in lateral lithology and structure, and may be present either underground or on the surface. A number of studies have been conducted to understand the influences of these structural features on mine stability and ground control planning (Peters et al. and Jones et al., 1986; Hill III, 1988; Molinda et al., 1991). In general, we can divided the structural features into two categories: the surface structural (linear or curvilinear) features may be faults, stream valleys, canyons etc., whereas the underground features usually refers to sandstone channels, paleochannels, clay dikes, 
faults, and etc. Field studies and numerical models have indicated that structural anomalies may play a major role in the stability of mines where they are present. For example, a strong correlation between roof failures and stream valleys has been shown in mining areas of West Virginia and Pennsylvania during studies conducted by many previous researchers (Molinda et al., 1991; Stankus et al., 1999). They concluded that roof stability is adversely affected by stream valleys due to the stress concentration zone under the stream valley. Unstable roof conditions may most often occur beneath the broader, flat-bottomed valleys than beneath the more confined, narrow-bottomed valleys. To identify those potential hazardous structures and record them correctly on mine maps has been one of the important responsibility of mine engineers and geologists. Traditionally, most large-scale structural features can be discovered by field investigation and core drilling. For the smaller complex underground structures, a number of advanced technologies have been developed to detect and analysis their locations and extensions. These methods include analysis of remote sensing data from satellites (Peters et al., 1986); the seismic tomography technique (Westman, 2003); and the Radio Imaging Method (RIM-IV) (Stolarczyk et al., 2003).

In addition to the geologic and structural conditions, geo-mechanical stress effects on the mine stability have gained great attentions over recent years. There were numerous instances where the horizontal stress has been a predominant factor adversely affecting mine stability (Hanna et al., 1986; Molinda et al., 1991; Stakus et al., 1999; Gadde et al., 2004) at certain mines. The thickness of overburden, the orientation and shape of the valley, topography, and the magnitude and orientation of the regional in-situ tectonic stress all influence the distribution of horizontal stress at seam level. In general, when the 
orientation of the maximum horizontal stress is perpendicular to the orientation of an entry, the entry would experience the minimum stability, while the entries with the same orientation as the horizontal stress would be under the most stable condition. Also, with increasing seam depth, the increasing overburden stress and stress interaction effects of multiple seams may became the prevailing influence factors. Intensive studies on seam stresses and displacements induced by various underground mining conditions have been conducted over the past years by researchers. A number of numeric methods have been developed and applied to analysis the stress influence of the mine stability successfully (discussed at section 2.5).

As we know, any of the factors discussed above may affect the mine stability. However, for a particular mine, some of the factors may dominate others and the degree of hazard of each factor varies from one area of the mine to another. So a comprehensive hazard factor which takes in consideration all critical factors and their respective degree of hazard will have great benefits to better understand any potential roof problems and produce a stable, safe mine design.

\subsection{Stability Mapping with Multiple Influence Factors}

It is well known by now that the roof stability is generally related to many different factors (Stankus et al., 2001; Riefenberg, 1994; Watts et al., 2000), and it often is a combination of geologic and geo-mechanical properties with each property having a different degree of influence on the final stability.

Generally speaking, for each particular mine and geologic situation, different types of influence combinations would be appropriate. Each mine typically has its own local stability criteria determined through mining experience. A particular mine's 
stability criteria may be a function of the thickness of a geologic layer, the nearness of a layer to the seam, the strength of a layer, the chemical composition of a layer, the magnitude of inter seam stresses, etc.

For instance, a specific mine may know that the roof stability linearly degrades as a shale parting in the roof thickens up to a maximum of two feet. Then the degree of hazard for the shale thickness stability factor can be determined by assigning a value of 0 at 0 feet in thickness and a value of 100 at 2 feet in thickness with following equations:

$$
\text { Stability Factor }=\left\{\begin{array}{l}
100 \times \text { Shale thickness } / 2 \text { feet, } \\
100 \quad \text { if Shale thickness }<2 \text { feet }
\end{array}\right.
$$

Another example is that the influence of the sandstone on roof stability is usually a combination of its thickness and the distance between its location and seam roof. So an appropriate influence factor combining both aspects needs to be determined.

So over the past years, researchers and mine engineers have been taking efforts to compose a comprehensive stability map by trying to combine the geologic and geomechanic factors into one quantitative stability factor in order to improve the understanding of roof stability. We will review some of these projects and discuss them in more detail below:

In 1994, the US Bureau of Mines researchers developed a hazard mapping system based on the combined effects of local and regional stresses, roof and floor quality, geology, faulting, etc. (Riefenberg, 1994). The approach used in their study was to first calculate the Coal Mine Roof Ratings (CMRR) for 10 borescope holes using unit ratings that were determined from roof exposures, excavation of overcast and faults. The CMRR 
over the whole study area (about 500 by 1000 feet) was estimated by kriging method, a geo-statistical analysis process. Figure 2.2 shows the estimated CMRR distribution over the whole study area. It can be seen form the figure that the CMRR value in the area fell between 57 and 77, which indicated a moderate to strong roof condition. At the same time, the seam stresses were calculated by using the numerical modeling program MULSIM/PC, a Boundary Element Method application. Figure 2.3 shows the simulated vertical stress for upper and lower seams.

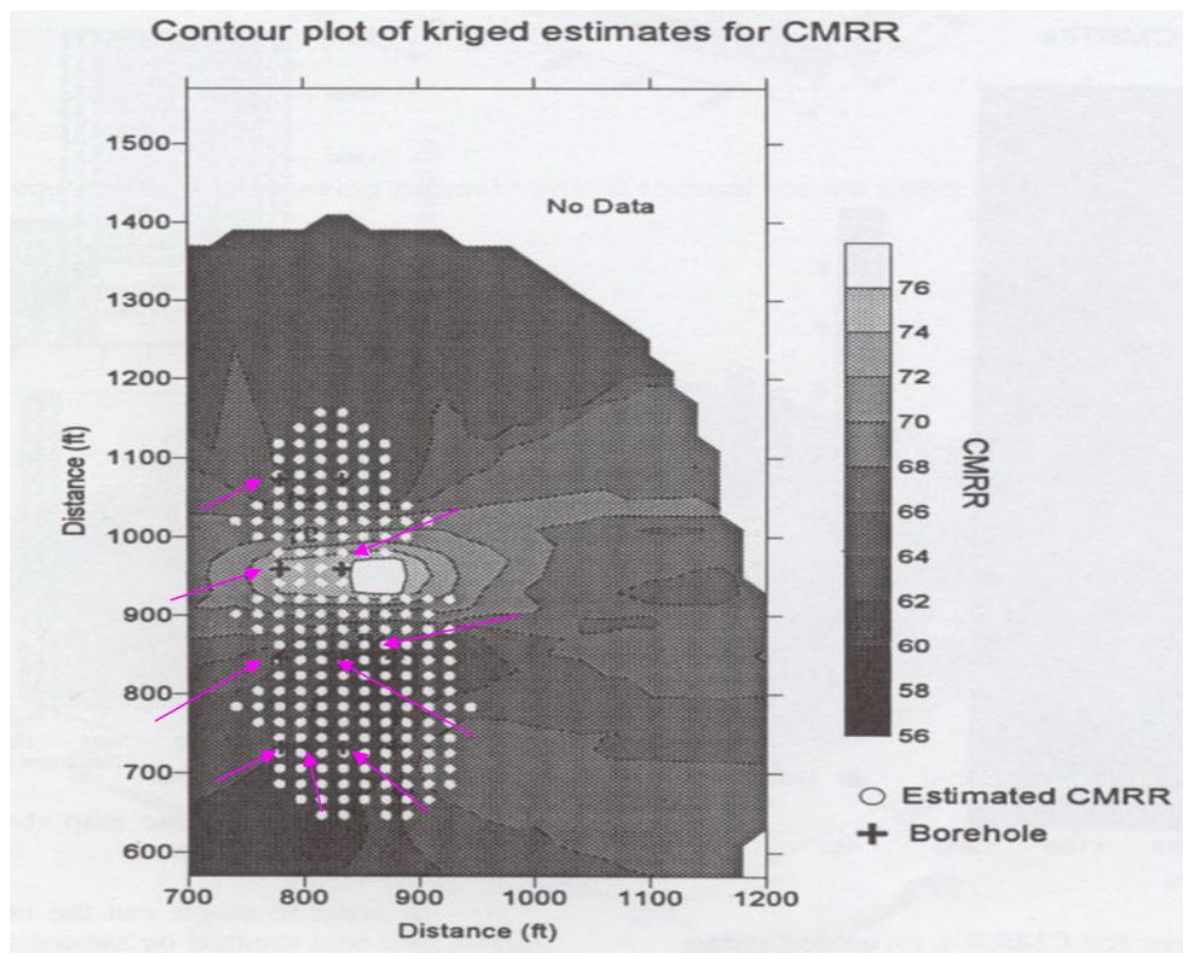

Figure 2.2. CMRR rating (after Riefenberg, 1994) 


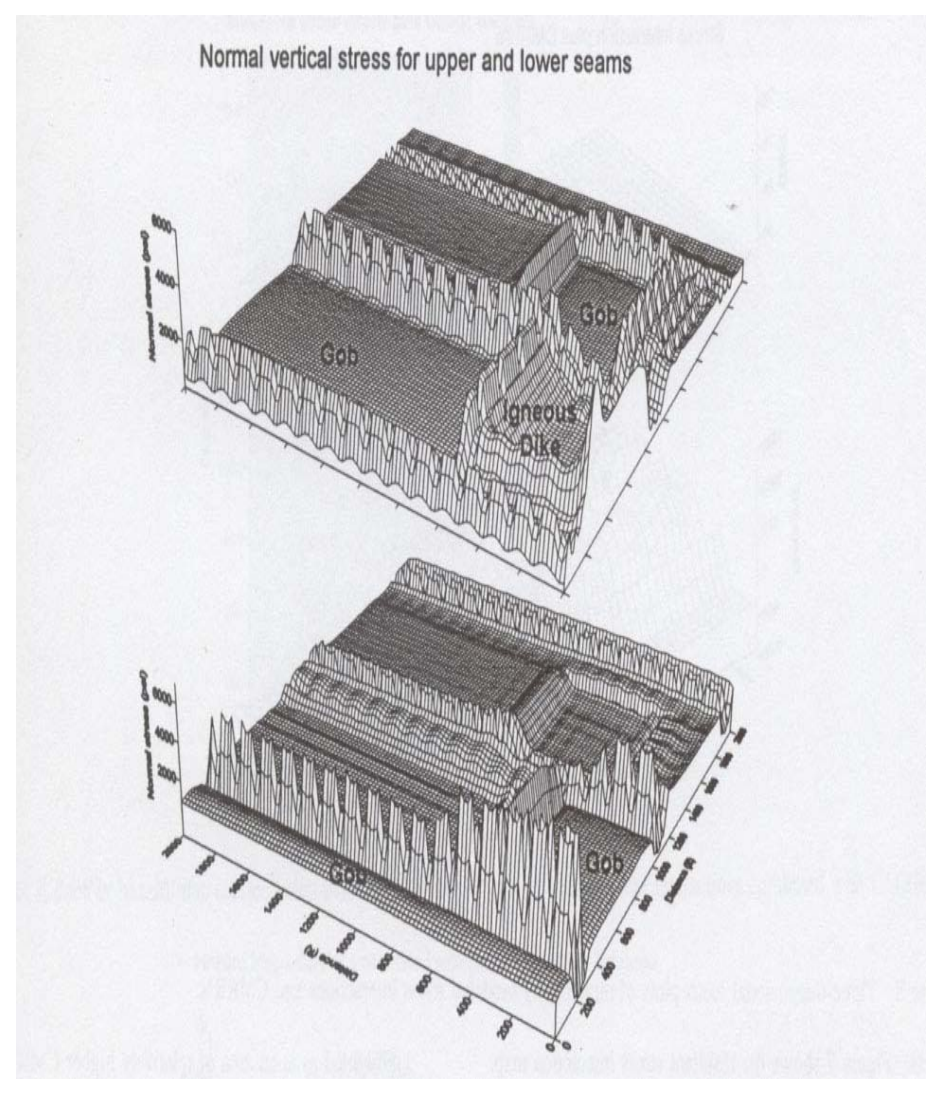

Figure 2.3. Vertical stress for upper and lower seam (after RiefenBerg, 1994)

The stress interaction was taken as the difference between the stresses calculated for the lower seam with, and without, the upper seam. The CMRR and stress values were imported into a 25 by 50 grid over the area (Figure 2.4 (a)). A hazard map was then created by combining these two factors with equal weights, after the values were scaled into a common $0-100$ index range (Figure $2.4(b)$ ). 


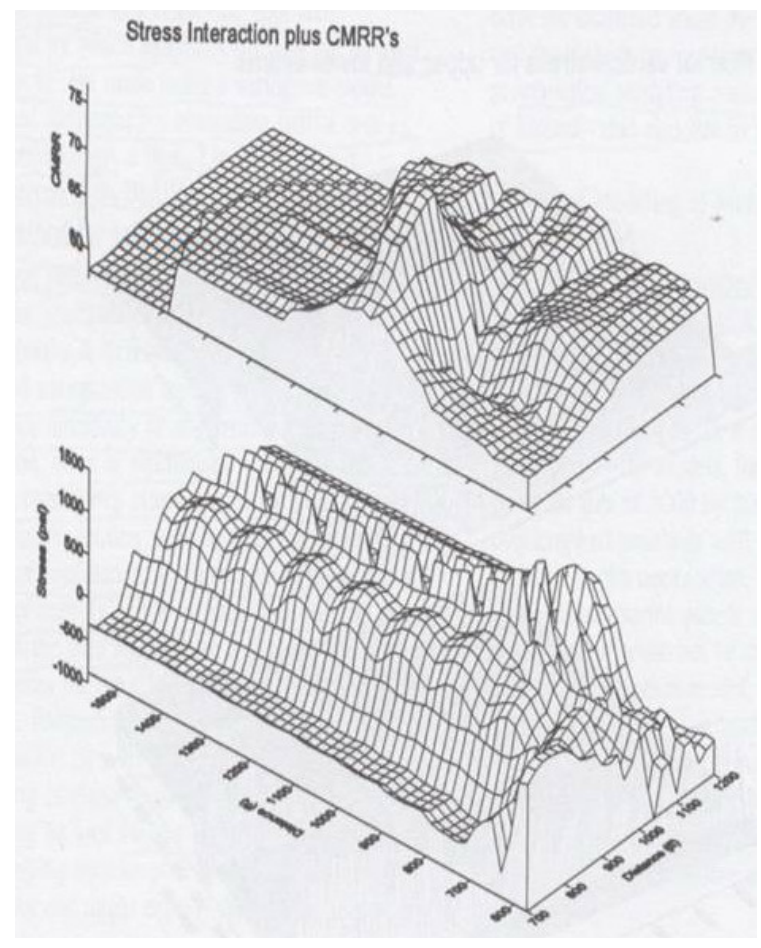

(a)

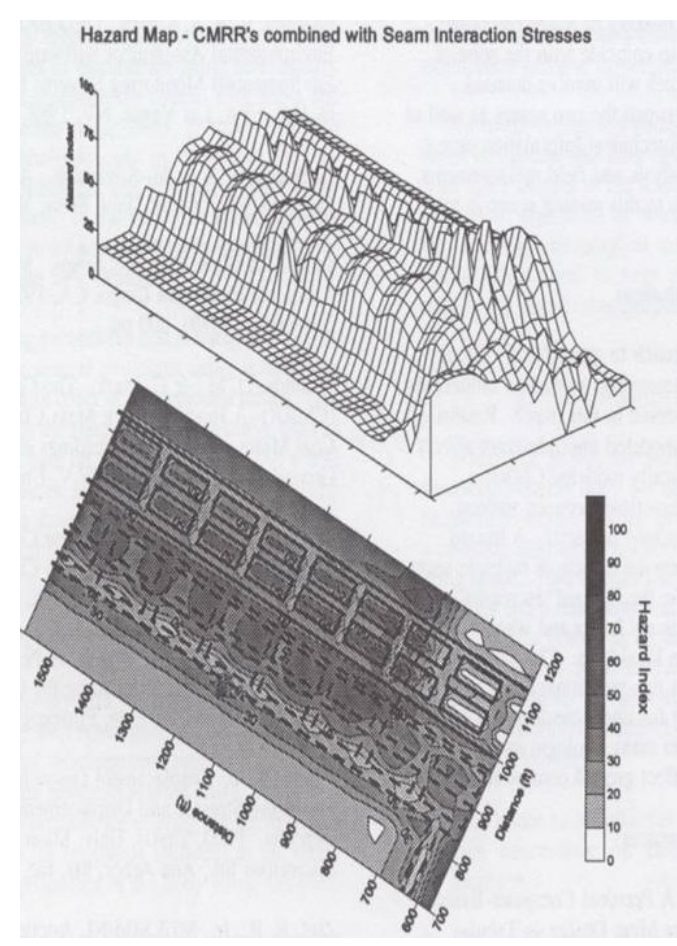

(b)

Figure 2.4. Individual factors and hazard map (after Riefenberg, 1994)

The limitations of their study were that only two parameters were incorporated and that limited samples was used in the CMRR estimation due to the difficulty of data collecting. However, this hazard mapping approach was proven to be a promising approach for understanding and delineating the interactions between many geologic and geo-mechanic factors that affect mine stability (Riefenberg, 1994).

Similar concepts have been also applied to more recent research projects by mine engineers. For example, a project done by Keystone Mining Services at Enlow Fork Mine of Consol Energy (Stankus et al., 2001). For this particular mine, based on the ground control experiences and roof fall history it was found that four factors had a significant 
influence on roof stability namely: sandstone channels, mica in the sandstone, tectonic stresses and stream valleys.

Among these factors, the factor related to sandstone channels actually is a combination of the sandstone layer thickness and the interval distance between the sandstone and coal seam. So, the sandstone factor was defined as the square root of the product of the sandstone thickness and the sandstone interval. In this case, these two individual factors have to be determined independently in advance. Figure 2.5 shows the distribution of the sandstone influence.

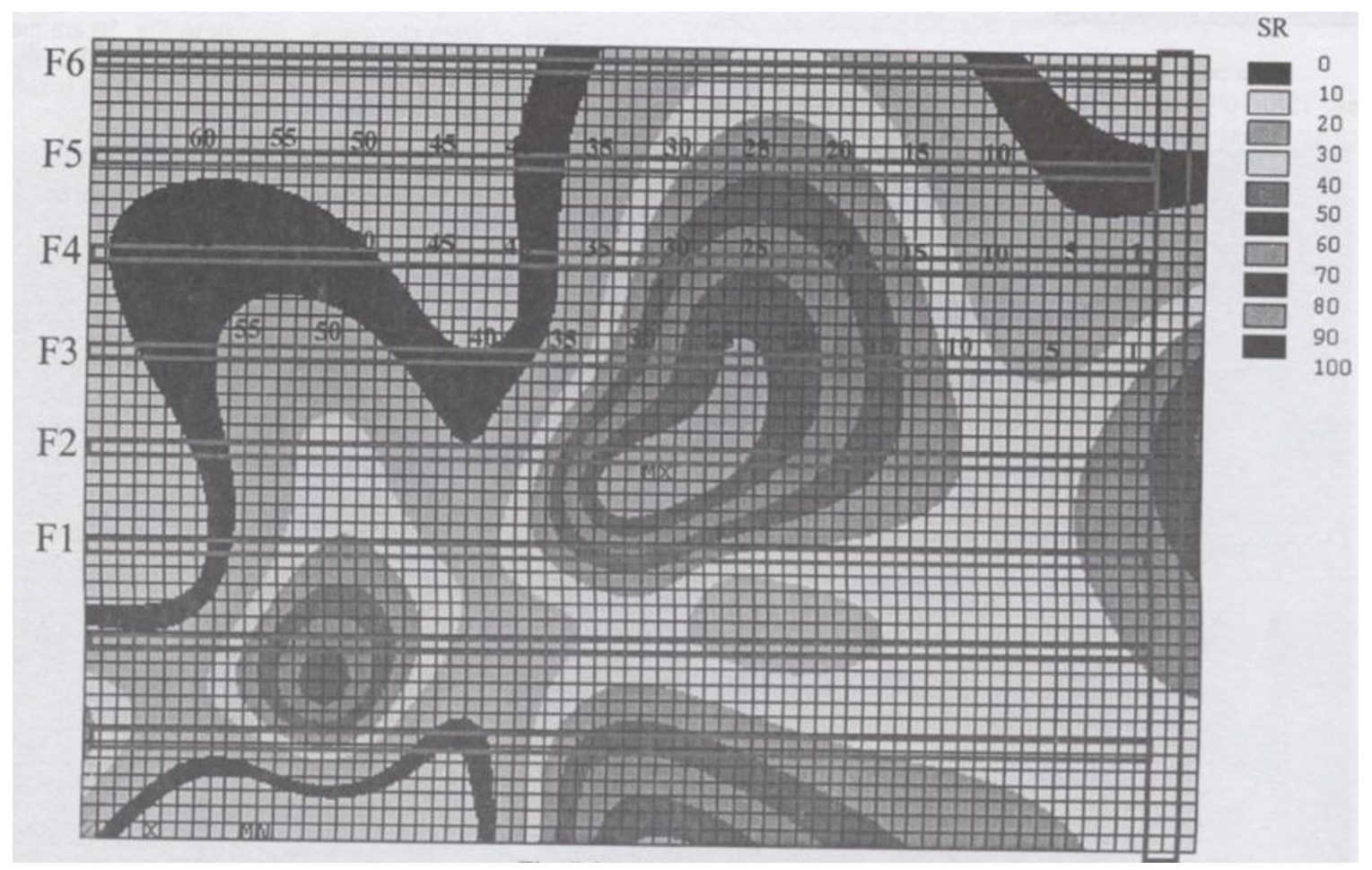

Figure 2.5. Sandstone influence over a longwall panel (after Stankus et al., 2001)

For the stream valleys, their influence areas are first identified by investigating the existence and influence distance from the bottom line of valleys (in this case $500 \mathrm{ft}$ was applied). These areas were considered as the high hazard area and were given the higher 
degree of stability influence. Figure 2.6 demonstrated the areas with and without stream valley influence.

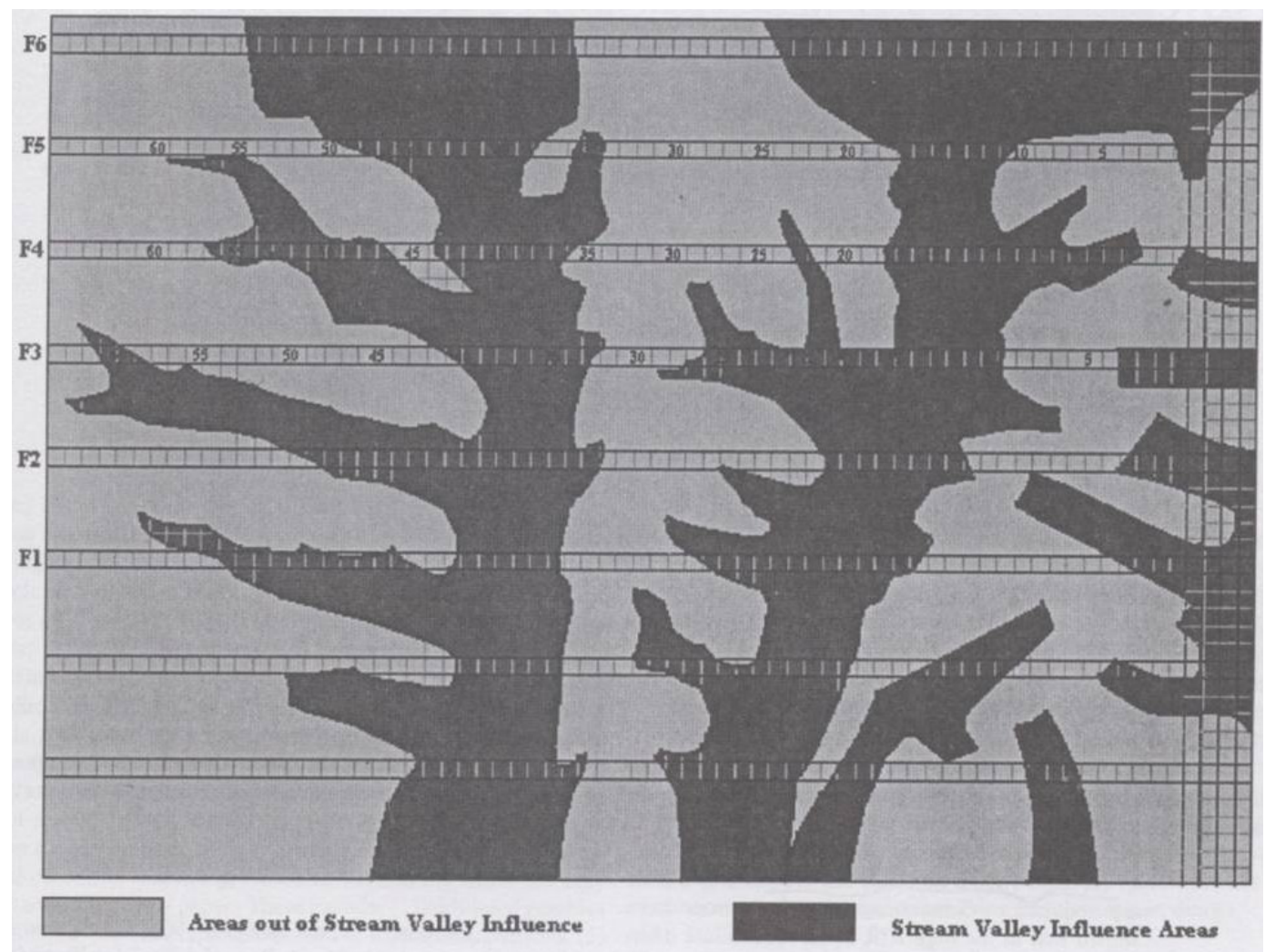

Figure 2.6. Areas with and without stream valley influence (after Stankus et al., 2001)

It was known at this mine, that the tectonic stresses were induced by geologic structures such as: synclines, anticlines and seam elevation changes, and that the roof was more often unstable within the influence area of the geology structures. To determine the stress distribution over the mine area, a Finite Element Method (FEM) model was built to simulate the seam condition. Figure 2.7 shows the calculated tectonic stress distribution. After identifying all these individual factors, a combined rating was defined as the equal weighted average of all four individual factors. Figure 2.8 is the final hazard rating over 
the area of study. The areas with the highest ratings are where an unstable roof condition may be expected, and supplement support may be necessary to be installed.

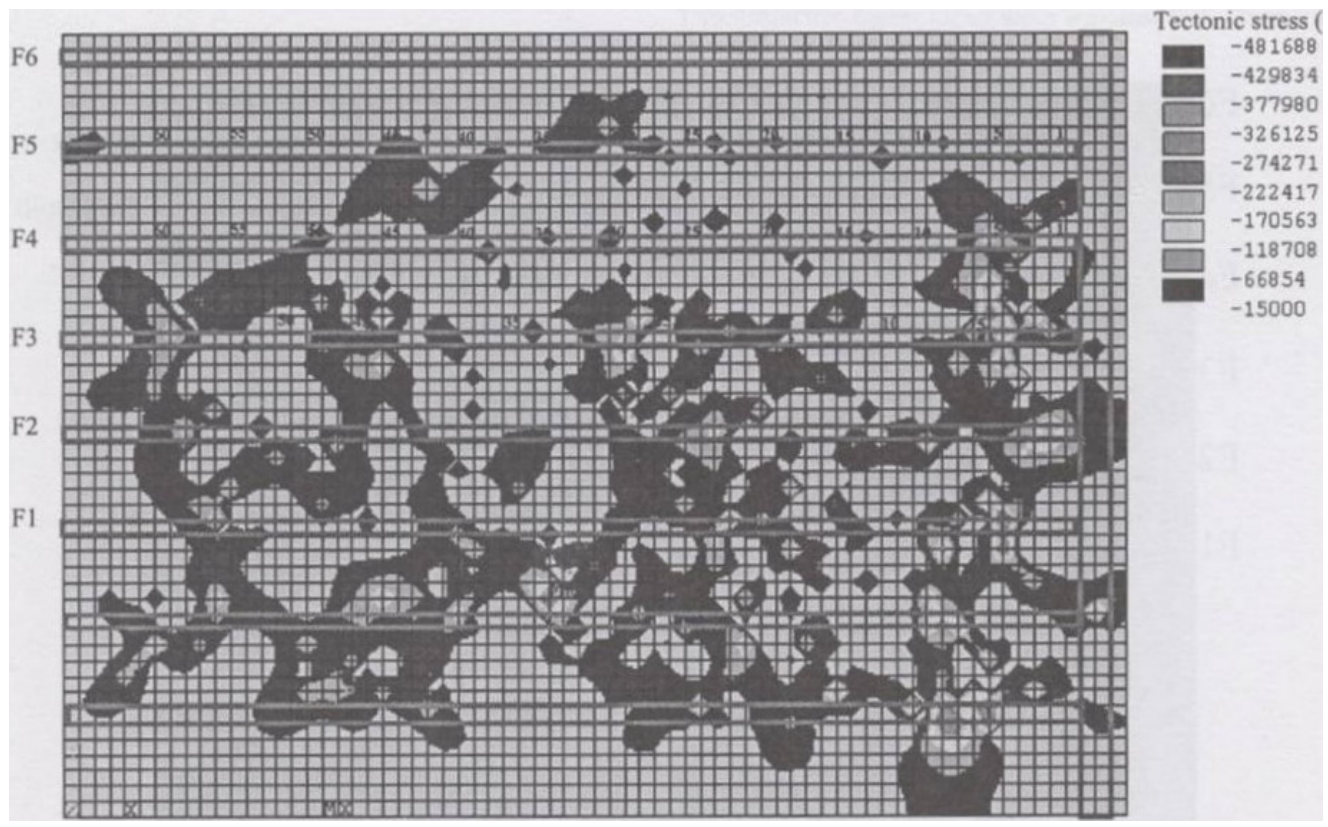

Figure 2.7. Tectonic stress influence (after Stankus et al., 2001)

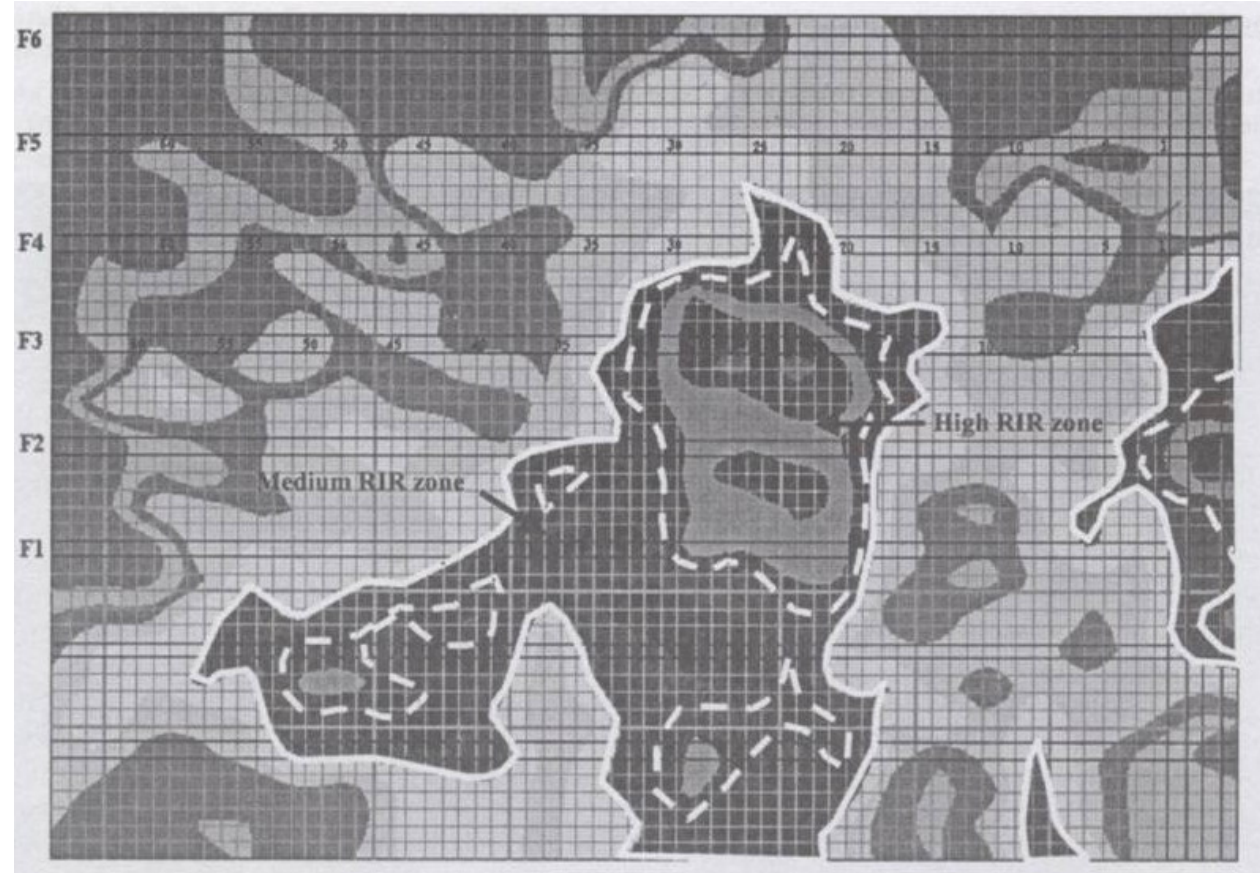

Figure 2.8. Multi-factor stability rating (after Stankus et al., 2001) 
After their work was applied to the support design and verified with field observations, it was concluded that the combined analysis with the geologic model and geo-mechanical model is a powerful approach enabling more accurate and cost-effective ground control design.

Another example of the integration of geology and geo-mechanical stress in ground control and mine planning has been applied to a limestone quarry. Mr. Dave Newman from Appalachian Mining and Engineering reported on a study for a multi-level underground limestone mine (Newman et al., 2001). In the study, geologic discontinuities including calcite veins and fractures, their orientations and conditions, as well as roof falls were inspected and accurately placed on the AutoCAD map of the quarry. Then numerical modeling with the LaModel program was used to simulate the stress interactions between the multiple levels. The combined effect from both the geology map and the numerical analysis was investigated carefully against the roof condition, although no single stability factor was derived. It has been concluded that a better understanding of roof stability was achieved through the combination of underground geologic mapping, rock strength testing and numerical modeling of the entire property (Newman et al., 2001).

From the analysis of the traditional geology mapping practices and current studies in multi-factor stability mapping, it can be summarized that a typical integrated stability mapping system will generally involve several basic procedures:

- Defining the critical geology, structure and stress factors which have the most influences on the roof stability. 
- Calculating quantitative values for each determined factor by using appropriate algorithms.

- Determining the individual stability factor by adopting a proper transformation function.

- Integrating the individual factors into an overall stability index by an appropriate weighting process.

- Mapping the stability index onto the mine map.

The integrated stability system discussed in the dissertation has been developed to meet the need of the analysis procedures (see Chapter 3).

\subsection{Numerical Methods on Mine Stability Analysis}

\subsubsection{Numerical Methods}

As discussed earlier, geo-mechanical stress has been considered as one of the important components that affect mine stability. With the advance of computer technology, a number of numerical techniques developed to help mine engineers and researchers better understand the nature of geo-mechanics have been proven to be very useful tools in modeling the mechanical state of underground structures under complex geometric and geologic conditions.

From a mathematical point of view, underground tabular ore mining problems belong to a class of problems known as boundary value problems, which are characterized by a region of interest enclosed within a boundary (Crouch et al., 1983). In general, the approximate numerical solution for solving these boundary value problems can be divided into two distinct categories: one requires approximating the solutions throughout 
the region and another only requires solving for the elements on the boundary of the region. The finite element and finite difference methods fall into the first category and the boundary element methods fall into the second.

Finite element and finite difference methods have been used to analyze a broad spectrum of ore excavation problems. In these techniques, the whole domain (region) is divided into a network of elements. The displacement solution at the nodes of the network is expressed in the governing differential equations, which leads to a system of linear algebraic equations with known nodal values at the boundary. The solution of this system of equations requires solving a large sparse matrix. Therefore, applying such a numerical technique to mining problems, especially for analyzing large tabular deposits is computationally time consuming and expensive in term of computer resources.

On the other hand, in the boundary element method, only the boundary of the region of interest needs to be divided into elements. The numerical solution builds on the analytical boundary solutions, while the analytical boundary solution can be obtained by solving simple singular problems with specified boundary conditions in advance (Crouch and Starfield, 1983). When this technique is applied for estimating displacements and stresses induced by extractions of tabular seam deposit, only the planar area at seam level needs to be discretized. Thus, this approach will result in a much smaller system of equations than finite element methods to solve the same problem, and therefore a relative large area can be simulated with the minimal amount of effort and resources. 


\subsubsection{MULSIM/NL}

Crouch (1983) illustrated three types of boundary element methods: the fictitious stress method, the displacement discontinuity method and the direct boundary integral method. For analyzing the broad displacements and stresses associated with the extraction of large tabular deposits such as coal, potash or other vein-type deposits, the displacement-discontinuity version of the boundary-element technique is generally the best modeling choice. In the displacement-discontinuity (DD) approach, the mining horizon is treated mathematically as a slot in the surrounding media (a discontinuity in the displacement field). With this simplification, only the planar area of the seam needs to be analyzed in detail in order to obtain reasonable stress and displacement values for the in-seam material. By limiting the detailed analysis to only the seam, the DD method provides considerable computational savings, and is able to efficiently calculate stresses around very large areas (a square mile or more) of tabular excavations.

The initial mathematics of the displacement-discontinuity method was first presented as early as the 1960s (Salamon, 1962; Berry, 1960). At that time, only hand calculations were available. The strict solution of the seam convergence distribution for complex excavation outlines was not possible by hand; and therefore, only the closedform of the equations for the displacements and stresses surrounding a single thin opening was initially used. However, a physical analogue was quickly developed which enabled the displacements in the seam to be measured in terms of the voltage distribution across an electrode in an electrolytic tank (Ortlepp and Nicoll, 1964; Salamon et al., 1964). This electrode was meticulously shaped like the excavation, and voltages (analo-

gous to the displacements) were tediously read using a vacuum tube voltmeter. The 
initial electrolytic tank analogue was then superseded by a more general, electricalresistance analogue, which used a three-dimensional grid of electrical resistors instead of the electrolytic tank (Cook and Schumann, 1965). The electrical resistance analogue also incorporated an easily modified "peg board" surface for representing the mining plan and allowed researchers to directly measure not only voltage, which was analogous to displacement, but also current, which was analogous to stress.

Very quickly, computer technology progressed and the advantages of a digital solution quickly replaced the analogue techniques (Starfield and Fairhurst, 1968). By the early 1970s, computer code was developed to replace all aspects of the analogue solution from calculating the seam convergence to numerically integrating the stress and displacements anywhere in the surrounding media (Deist et al., 1972). This initial displacement-discontinuity code was known as the Mining Simulator or MINSIM for short. (A much-updated version of MINSIM is still used in South Africa). A few years later in the United States, Sinha's doctoral thesis (1979) presented the basics of three programs: one for tabular seams near the Earth's surface, one for multiple seams in an infinite elastic space (MULSIM), and one for piece-wise tabular seams in an infinite elastic space. The U.S. Bureau of Mines then took Sinha's program, MULSIM, and implemented a full-featured, public-domain, displacement-discontinuity program they called MULSIM/BM (Beckett and Madrid, 1988). The original MULSIM/BM code was used in several projects with fairly good results (Kripakov et al., 1988), and was eventually upgraded to MULSIM/NL by the addition of: non-linear seam materials (Figure 2.5.1), energy calculations, and multiple mining steps (Zipf, 1992a, 1992b). Figure 2.9 shows the 6 in-seam material models used in MULSIM/NL program. The 
initial displacement-discontinuity programs MINSIM, MULSIM, MULSIM/BM were written using the FORTRAN programming language and were run on the original mainframe computers. The MULSIM/NL started on mainframes and workstations, but was quickly ported to the personal computer as soon as PCs became popular in late 80 s. Recently, MULSIM/NL has been further updated by researchers from West Virginia University and run on the MS Windows environment and shares the same preprocessor and postprocessor with the more advanced DD program, LaModel (see Figure 2.9).

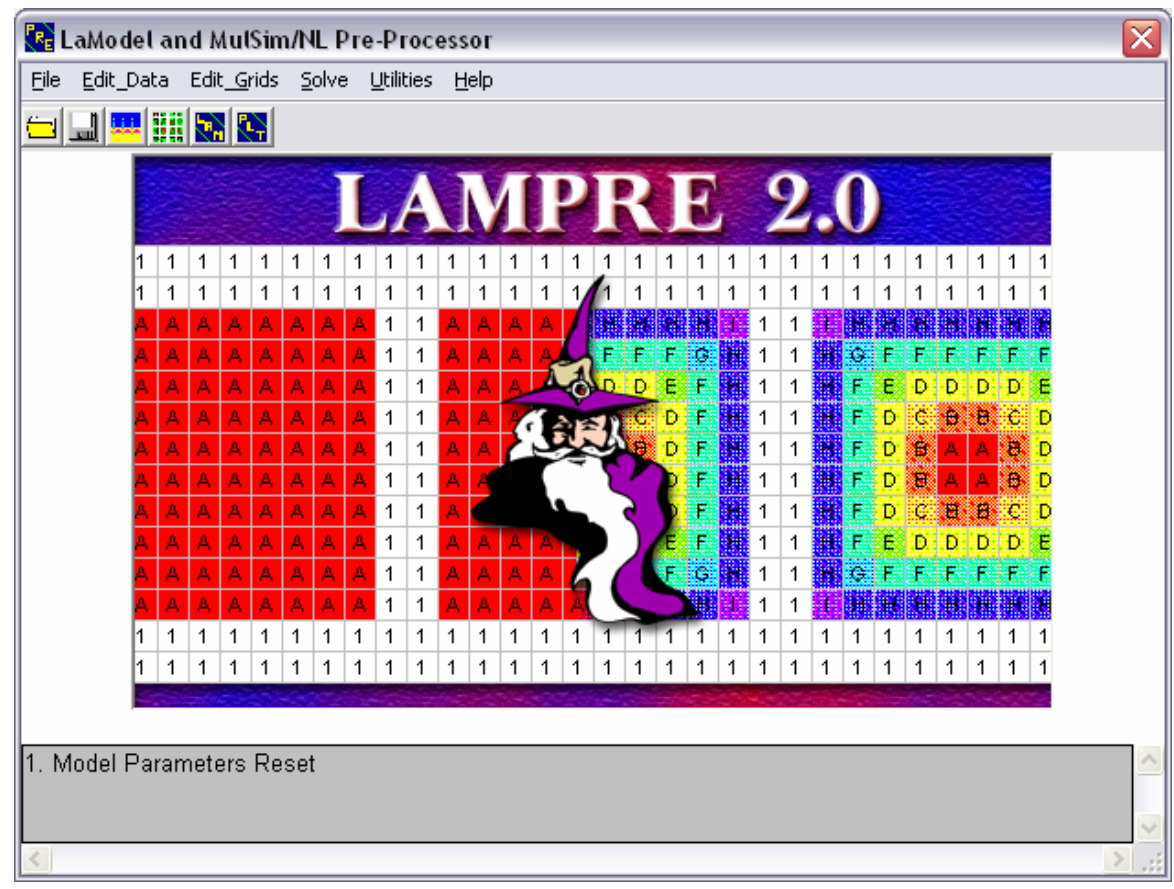

Figure 2.9 MULSIM and LaModel share preprocessor 


\subsubsection{LaModel and its Recent Development}

In order to increase the accuracy of the stress and displacement calculations for stratified rock masses, laminations were added to the overburden in the displacementdiscontinuity method to create the LaModel program (Heasley, 1998). Also, the ability to input a variable topography was first introduced with the LaModel program. The LaModel program (and the accompanying pre-processor, LamPre, and post-processor, LamPlt) was the first of the DD programs to be developed in the more modern objectoriented, visual programming environments.

In comparison to MULSIM/NL, LaModel implemented the same in-seam material models such as linear-elastic, strain-softening, elastic-plastic, strain hardening, bilinear hardening and linear elastic gob (Heasley, 1998 and Zipf, 1992). However LaModel also added much more advanced features such as:

- Laminated overburden model instead of a homogeneous elastic mass overburden (see Figure 2.10).

- Stress and displacement analysis for broad areas

- Faster definition of seams grids and mining steps with ease-to-use preprocessor LamPre (see Figure 2.11)

- Subsidence prediction

- Topography effects

- Up to 26 in-seam materials

- Graphic output by the post-processor LamPlt. 


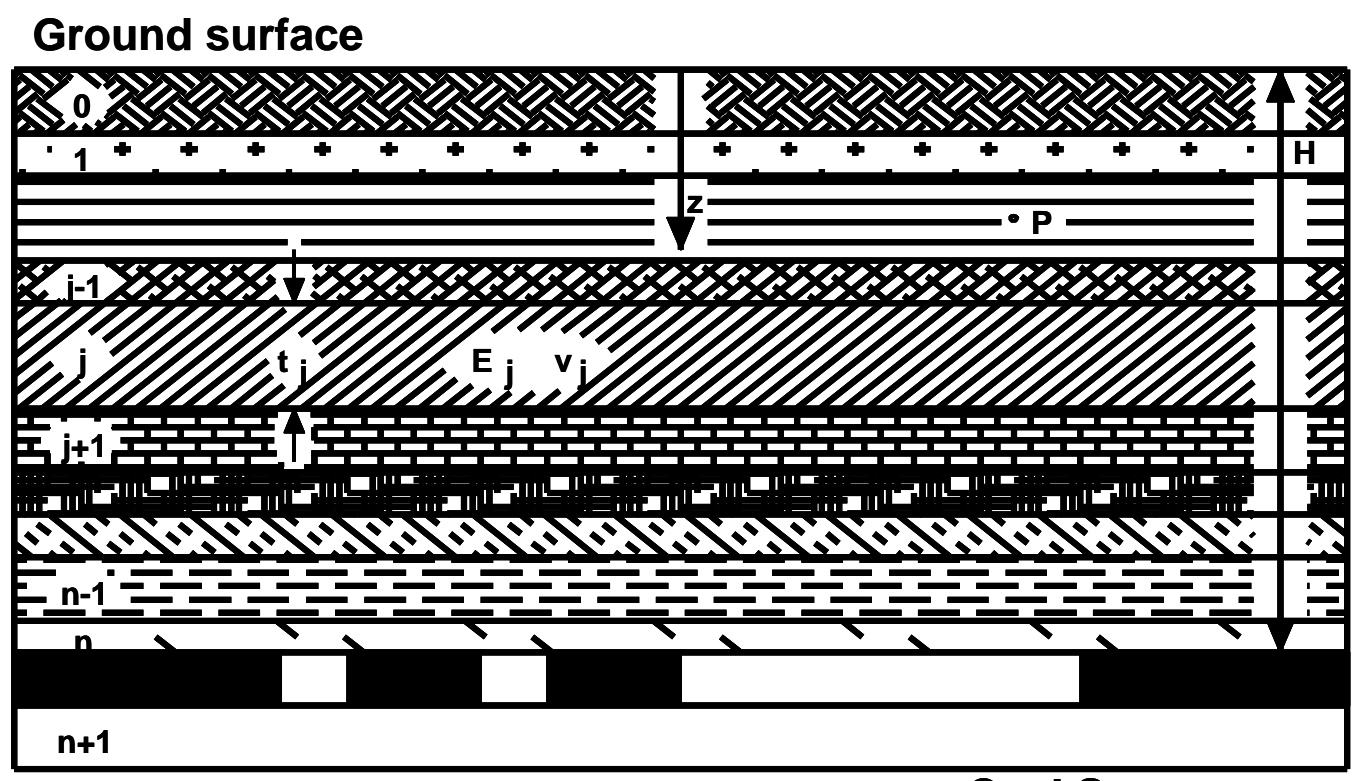

Coal Seam

Figure 2.10. Laminated overburden model

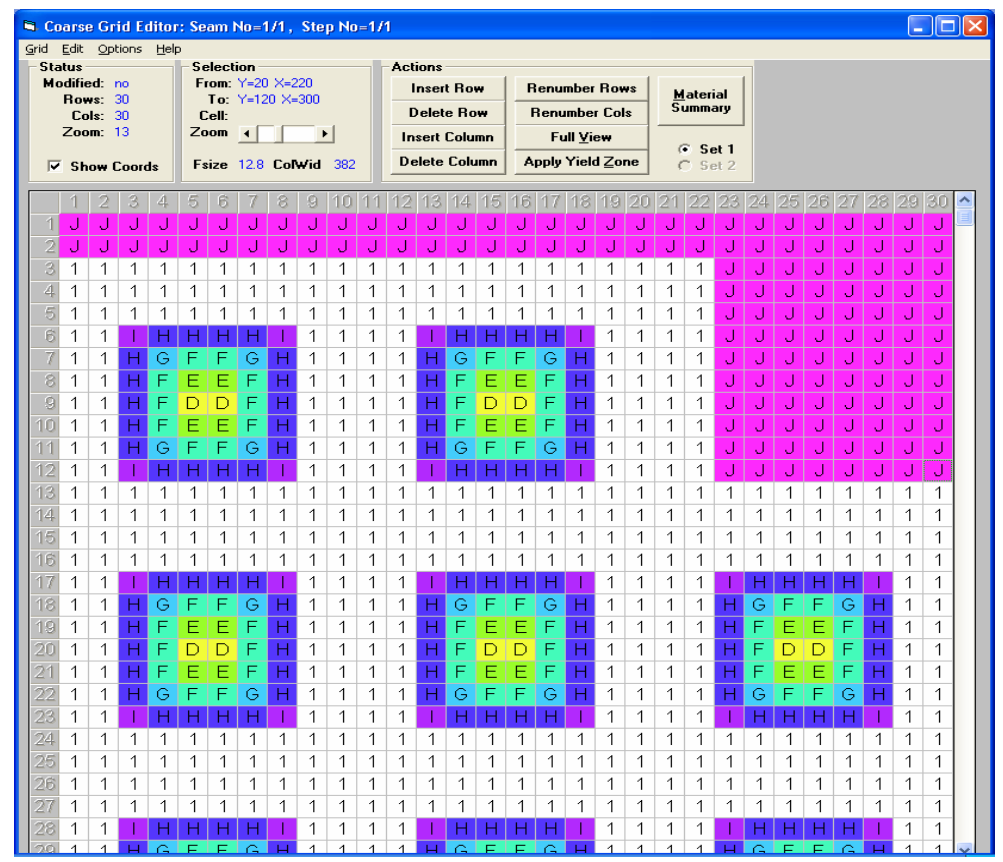

Figure 2.11. LamPre grid editor

Among the three components of LaModel, the preprocessor LamPre is a powerful tool to manipulate the input data (geometry and mechanics) for both LaModel and 
MULSIM. It also integrated with a visual grid editor to define and handle complex mine grids with specific cell properties.
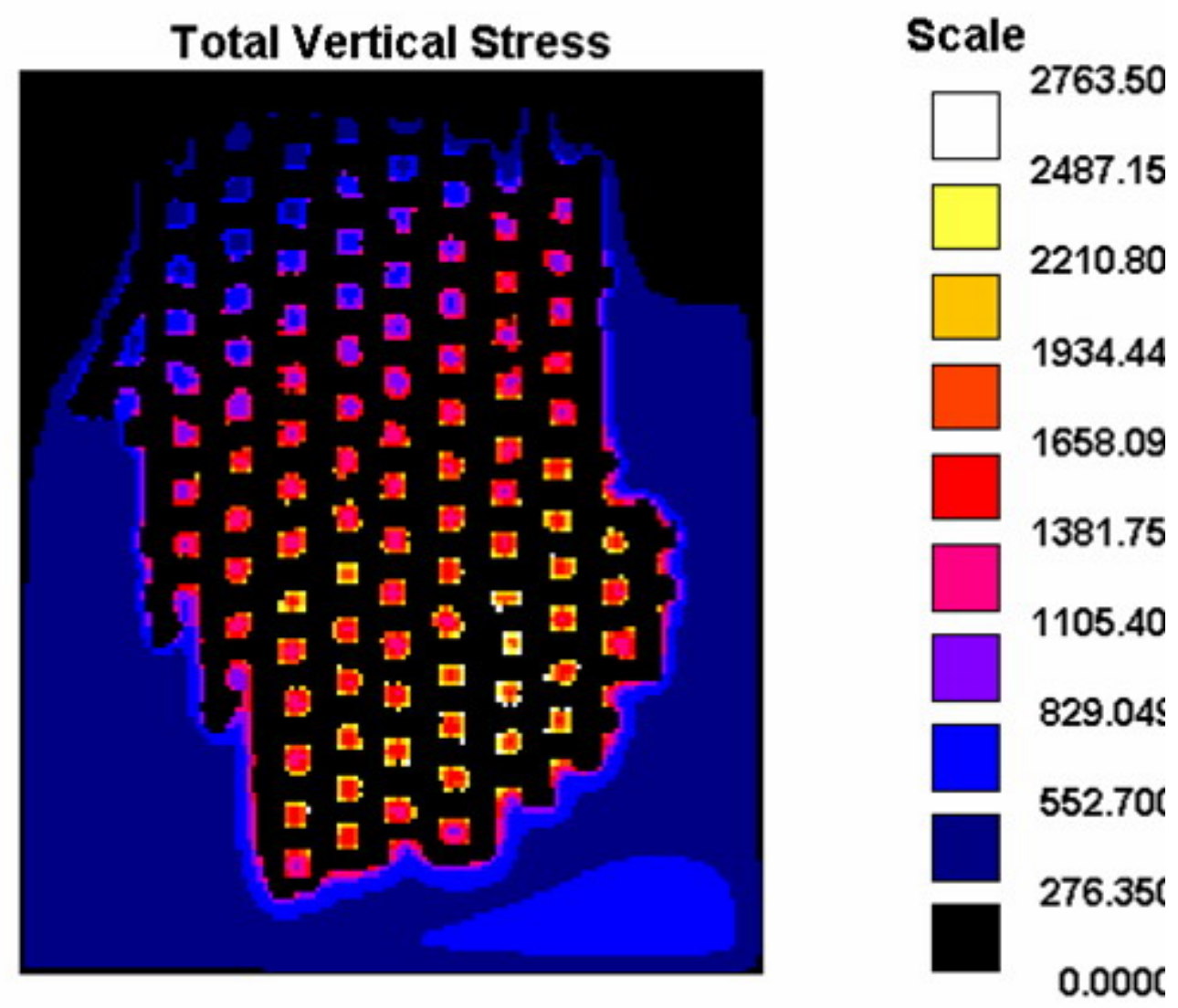

Figure 2.12. A typical LaModel vertical stress output

The typical output for a multiple-seam LaModel simulation includes: seam convergence, total vertical stress (Figure 2.12), surface effect stress, multiple seam stress and overburden stress. These basic outputs provide most of the necessary geo-mechanical information for a mine stability analysis.

The ability for more realistic multiple-seam simulation and the improved graphic user interface lead to a rapid acceptance of LaModel by the mining industry, research facilities and academic institutes. A lot of successful applications of LaModel can be found in the 
literatures and technical conferences throughout North America, Australia and South Africa.

During the intensive applications in the industry, some new requests have been developed by end users. In response to these requests, a number of enhancements to LaModel have been completed in order to allow faster, more complete and complex analysis. The latest enhancements include:

- To increase the speed of the automatic grid generation from complex irregular mine layouts, faster algorithms for generating the mine grid and topographic grid within the AutoCAD visual interface were implemented. These new algorithms have reduced the calculation time from hours to minutes.

- To help automate and simplify the mine map correction process, a couple of utility routines for automatically joining pillar polylines, closing pillar polylines and deleting duplicate pillars were also developed.

- To help increase the usability of the LaModel program, an automatic safety factor calculation routine was implemented and the resulting safety factors are one of the standard outputs from the program.

- Also to help increase the usability of the LaModel program, a subsidencebased strain calculation was implemented. This option now allows the user to consider both multiple-seam stress transfer and subsidence-based strains in an under-mining situation.

- Finally, in order to accommodate user's desires and to utilize increased computer capabilities, the maximum allowable size for the LaModel grid was increased from $400 \times 400$ to $1000 \times 1000$. 
Figure 2.13 shows a zoomed-in view of a mine grid generated from irregular mine pillars using an AutoCAD mine map. This realistic grid undoubtedly will result in a more accurate stress and displacement analysis.

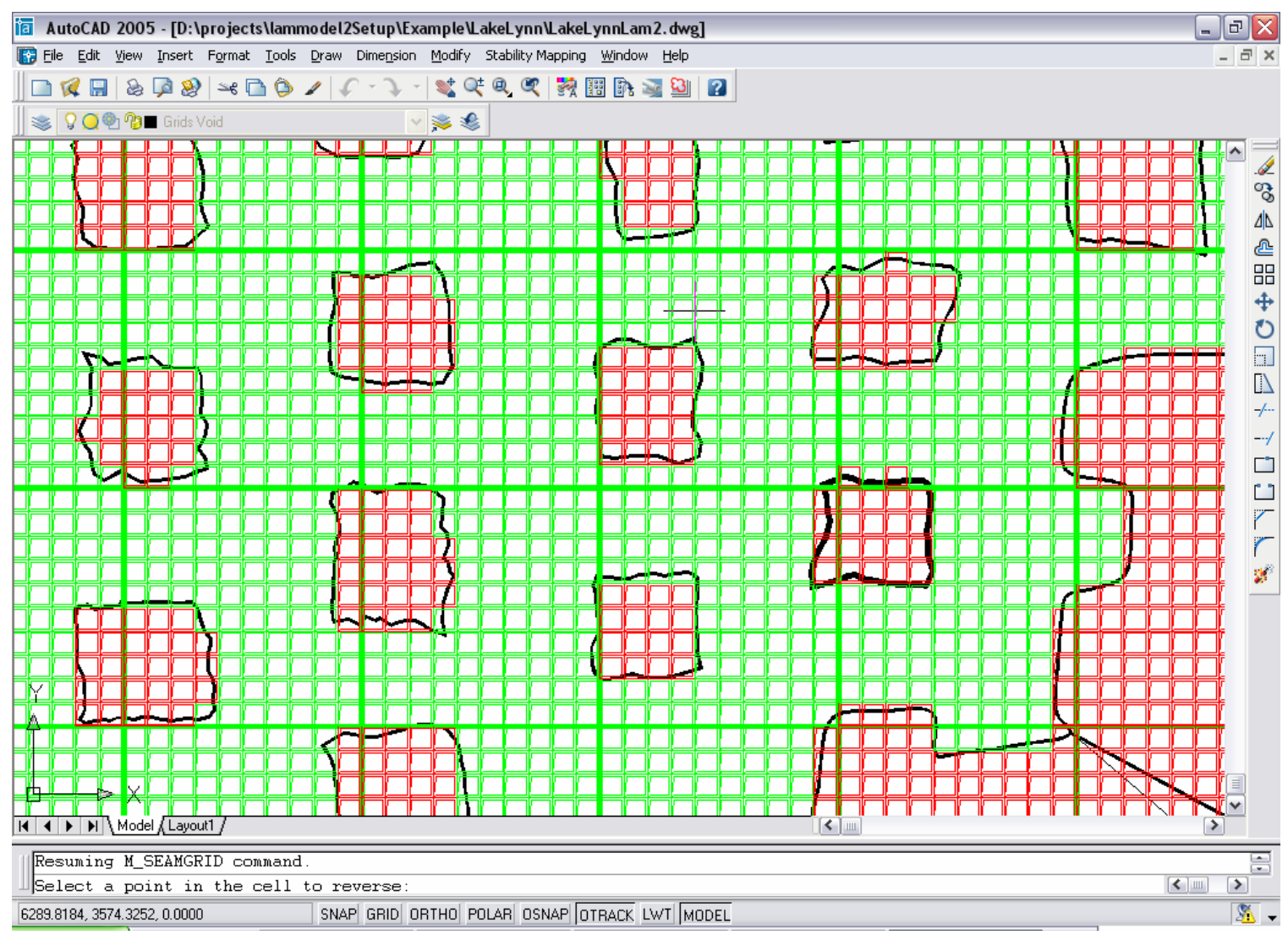

Figure 2.13. A close-up view of automatically generated mine grid in AutoCAD

\subsection{AutoCAD Customization and Programming}

One of the most important reasons that AutoCAD software is used so widely in industries is due to its excellent flexibility and extensibility. From very beginning of its development, AutoCAD had been providing the possibility of customization by exposing the embedded AutoLISP program language and exchangeable $\mathrm{DXF}^{\circledR}$ file format. With the latest versions, many advanced extension methods have been published for users to 
employ in customizing AutoCAD from general drawing software into a professional design package in their specific fields. These include:

- $\quad$ DIESEL - String Expression Language

- Command Scripts

- $\quad$ AutoLISP (Visual Lisp)

- AutoCAD VBA - Visual Basic for Application

- Autodesk Development System (ADS) - early C/C++ API

- ActiveX Automation - Based on Microsoft COM technology

- ObjectARX -- AutoCAD Runtime eXtension, the C++ Object Oriented API

All these interfaces to AutoCAD have their own properties along with their specific capabilities and limitations. Therefore, when one comes to the point of developing an application within AutoCAD, the best choice of which approach to use has to be made based on the nature of the proposed project and the requirements for integrity and efficiency. Among the possible customization methods, AutoLISP, ActiveX and ObjectARX are the most functional and suitable for developing complex applications, but they all have their respective advantages and disadvantages as discussed below.

\subsubsection{AutoLISP language}

LISP is an acronym for LISt Processing, a simple, yet deceptively powerful computer language. It was developed in the 1960's and was first used to control powerful telescopes for astronomy. Because its main strength was to handle the coordinates of a point in space, and this ability can be used to create a drawing with a symbol placed at the coordinate position, it soon became a very sensible choice for computer aided design 
where we are dealing with the placement of entities in drawings at very tightly defined coordinate positions.

AutoLISP is Autodesk's implementation of the lisp programming language. The major advantage of using AutoLISP is that it enables the creation of new commands in AutoCAD. We could, for example, easily create a new command which, when implemented, might perform the function of reading the line of text and placing a tree symbol at the coordinates specified in the file. AutoLISP has been embedded within AutoCAD from very beginning. AutoLISP routes can be created by using any simple text editor and then the routine can be loaded into AutoCAD to execute.

However, AutoLISP is an interpreted language with strict unconventional syntax of many matched, nested parentheses and limited access to the internal AutoCAD database and external function libraries. Therefore, AutoLISP is able to produce quick and fine solution only for a short, simple purpose, real-time or interactive application, but it is not suitable for complex, data intensive and high performance applications.

\subsubsection{ActiveX Automation}

AutoCAD ActiveX Automation is based on the Component Object Model (COM) interface technology introduced by Microsoft. The purpose of the COM technology is to provide standards and specifications required to create components that can interact with each other regardless of the computer languages and tools used to develop them. AutoCAD ActiveX Automation lets one access and control AutoCAD objects from a third party application such as Visual BASIC, Visual LISP, Microsoft Excel and other programs incorporated with the COM technology. AutoCAD Automation objects 
represent precise parts of AutoCAD such as all graphical objects (lines, arcs, etc.), style settings (line types, dimension styles, etc.), organizational structures (layers, blocks, etc.), views, view ports. Even the drawing document and the AutoCAD application itself are considered as objects. Automation objects make "methods" and "properties" available to the external programs. Methods are functions that perform an action on an object and properties are variables representing the states of an object.

Microsoft VBA (Visual BASIC for Applications) is an object-based programming environment accessible inside AutoCAD. VBA provides full Visual BASIC language syntax, the new forms package, and support for ActiveX controls. It also supports integration with other applications that use VBA, such as Microsoft Office. The VBA programming environment is relatively easy to learn and use. Its rapid user interface design tools can help develop user friendly application very quickly.

However, the main disadvantage of ActiveX automation is its low efficiency. An application can only access AutoCAD database through the COM object interfaces, attributes and methods, which are exposed to outside applications. Any query and edit command has to be sent to related object instances, which then invoke appropriate functionality defined in the object to communicate with the AutoCAD database. The result is returned to the COM object first before coming back to the application. Thus, when an application has intensive interaction with the AutoCAD database, this communication bottleneck of ActiveX automation can greatly limit its speed performance. 


\subsubsection{ObjectARX Programming}

ObjectARX (AutoCAD Runtime eXtension) is a new C++ API environment developed and provided by Autodesk, which provides an object-oriented applicationprogramming interface (API) with AutoCAD system. ObjectARX was introduced in AutoCAD Release 14 (1997). This release virtually opens up the internals of AutoCAD for building customized applications within AutoCAD. Using ObjectARX libraries, application developers can take full advantage of AutoCAD's open architecture, directly access AutoCAD database structures, the graphics system, define native commands and get notified on specific AutoCAD events.

Applications built in the ObjectARX environment typically run faster than those in the AutoCAD Development System (ADS, an old C style development interface before AutoCAD v14), COM and AutoLISP. An old style ADS program results in a separate executable file (EXE or EXP) that can be invoked from AutoCAD. An ObjectARX application, on the other hand, is a Dynamic Link functional Library (DLL) that shares AutoCAD's address space and makes direct function calls to AutoCAD, avoiding the costly performance and system overhead of inter-process communication (IPC) calls with COM. However, since ObjectARX applications share the same memory address space with AutoCAD, AutoCAD may go crash if the ObjectARX application does not handle exceptions properly. Figure 2.14 illustrates the relationship between these development varieties with AutoCAD. 


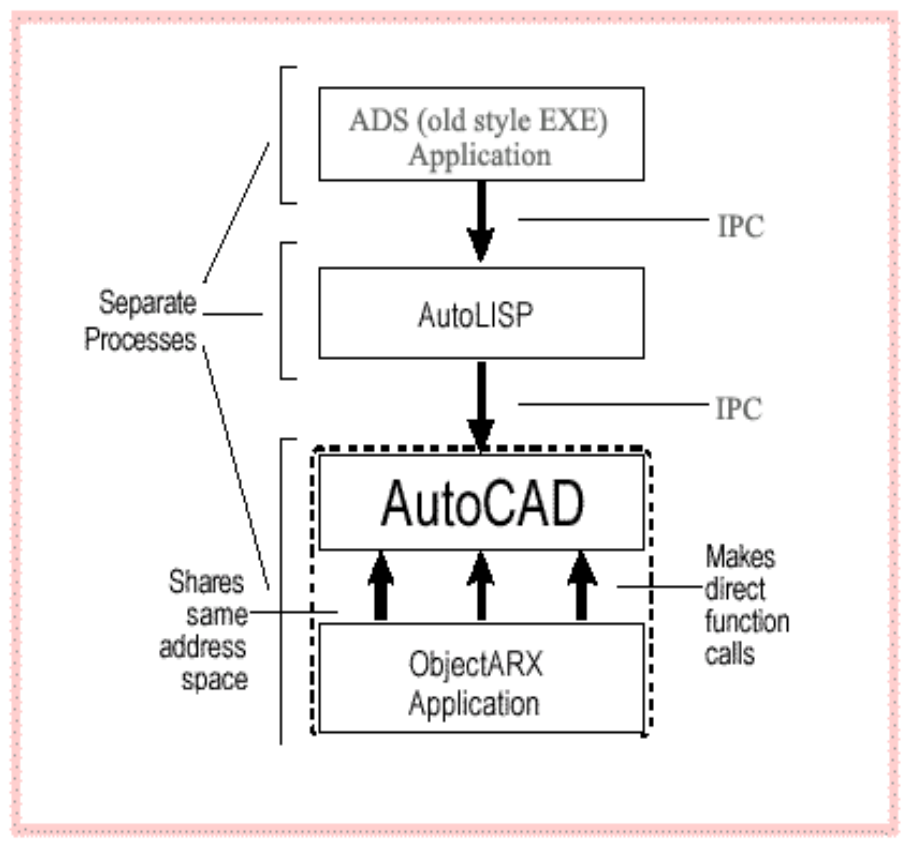

Figure 2.14. ObjectARX shares same address space with AutoCAD

ObjectARX allows applications to create intelligent design objects as custom entities, which become part of the AutoCAD database. Although a custom object usually composed of AutoCAD primary drawing types such as points, lines, etc., it can have user designed properties which may correspond to the characteristics of the object it represents. An intelligent custom object as a whole entity can have the ability to respond directly to editing commands, displayed according to their inherent characteristics. This inherent feature allows the developer to explore the possible extents of using real-world objects. These custom entities created using ObjectARX are virtually indistinguishable from built-in AutoCAD entities. Also, the new classes added in the ObjectARX application development environment can be exported for use by other programs. It is also possible to extend the ObjectARX protocol by adding functions at run time to existing AutoCAD classes. 
Using ObjectARX, one can not only customize AutoCAD, but extend it to where AutoCAD becomes just the base for a new application or product. Autodesk has led the way by highly advanced products such as Mechanical and Architectural Desktop built on top of AutoCAD using ObjectARX. Thus ObjectARX is rapidly becoming the first choice for serious application development in the AutoCAD environment. 


\section{Chapter 3. Design of Integrated Stability Mapping System}

\subsection{General}

As an integrated solution for stability mapping analysis, the integrated stability mapping system requires functionalities covering all aspects of the analysis procedures from data preparations, automatic information processing and transformation to final stability index composition and mapping. Many times, an analysis may need to go through a certain time period due to unavailability of data or may need to repeat one previous step because of changes of one or some parameters and data applied in that step. Therefore, an ideal structure for the system would be an open component-based architecture consisting of a system of comprehensive and independent functional modules that share the same data format.

In this chapter, a detailed structure of integrated stability mapping system is discussed. The requirements and problems inherent with various input data are also examined. Finally the component modules of the system are explored and classified.

\subsection{Structure of Integrated Stability System}

Based on the requirements for stability mapping analysis and characteristics of the system, an integrated stability mapping system is constructed in this dissertation using the structures as illustrated in Figure 3.1. 


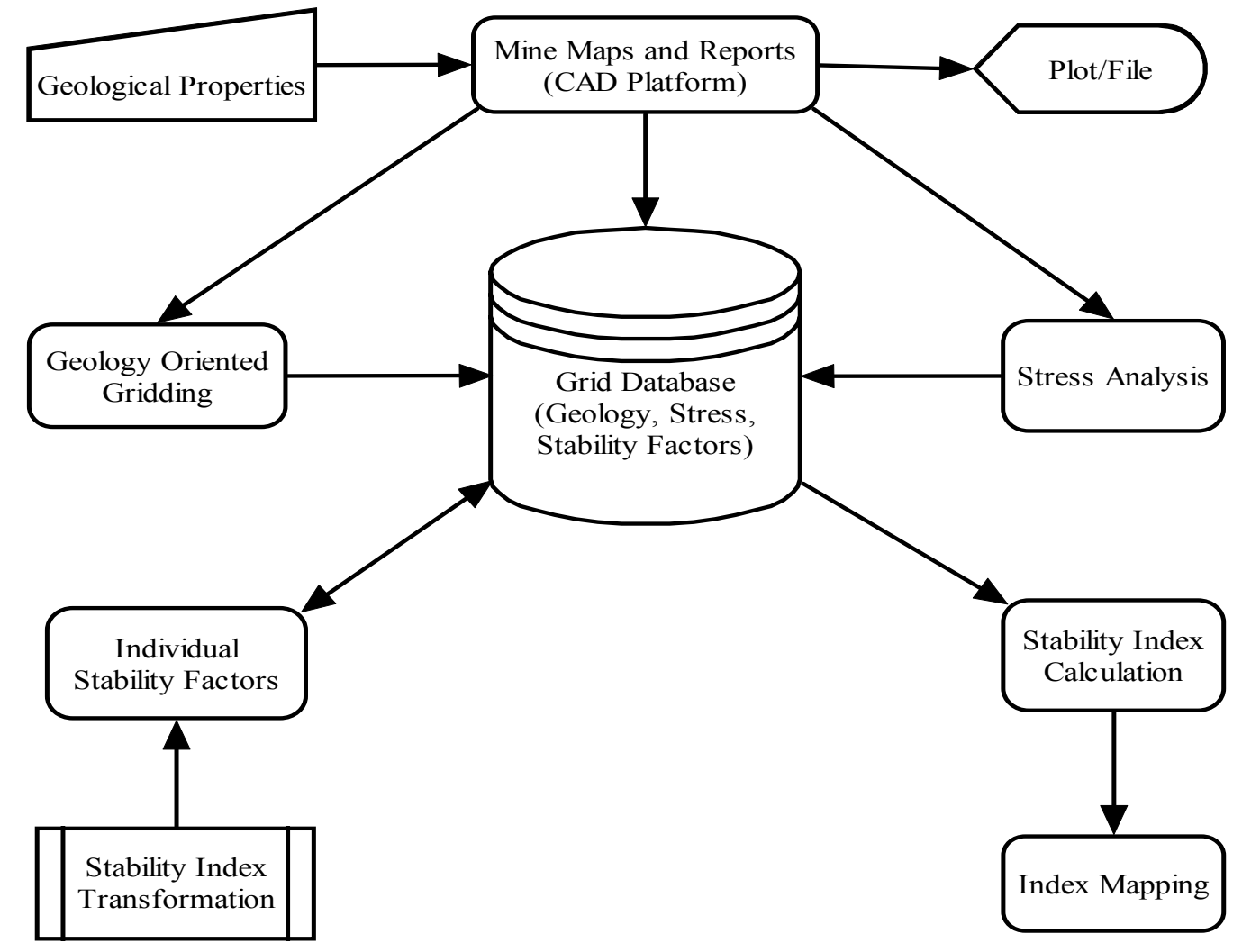

Figure 3.1. Schematic structure of the integrated stability mapping system

In the design of the integrated stability mapping system, it is assumed that the necessary geologic data are available in some AutoCAD compatible. The AutoCAD drawing software has been chosen as the basic underlying mapping environment because of its dominance in the operating coal mines in the US, its opening database specifications and its powerful capability for extension and integration (see Appendix C). It means that the developed functional modules in stability mapping system will access the information directly from AutoCAD database.

The basic data structure is a rectangle grid which covers the area of interest, and each element represents an area with a constant value of the properties. All calculated quantitative values and stability factors will be saved into grid files. In other word, these 
grid files form a gridded geologic and geo-mechanic database, and all of numerical values of critical influence factors in geology, geo-structure and geo-mechanics, along with the individual stability factors and final index will be stored in the database. Due to the variety of the influence factors and their data sources, a number of individual functional modules (geologic and stress modules) will be developed to calculate the quantitative values with different kinds of input data and information. The transformation module will implement various approaches to convert these quantitative values into the same and comparable scale. The stability index modules facilitate the composition and mapping of the integrated stability index. It can be seen that these independent modules will serve different purposes at various mapping steps. For instance, the geology and geostructural data can be imported directly from a geology model, or generated from a mine map by the geology-oriented gridding modules. The geo-mechanical factors will be created by the developed stress analysis modules. After the gridded data are available in the database, the individual stability factors can be determined by either predefined transformation functions or user-defined transformation functions. Then these individual factors will be calculated into one single stability index by a weighted average method or a user specified combinations. The final index can be plotted over the entries of the mining layout map.

In general, the procedure of a stability analysis can be performed in various sequences as long as the required input data are available. However, a typical stability mapping analysis would generally go through the following procedures (see Figure 3.2):

- Define and input the critical geologic, structural and stress factors which have the most influences on the mine stability. 
- Calculate a numeric grid for each critical factor.

- Determine an individual index for each critical factor (transformation)

- Compose the overall stability index (ex. Weighting the individual indices )

- Plot and analyze the overall stability index on the mine map.

- Re-evaluate critical factors and weightings as necessary.

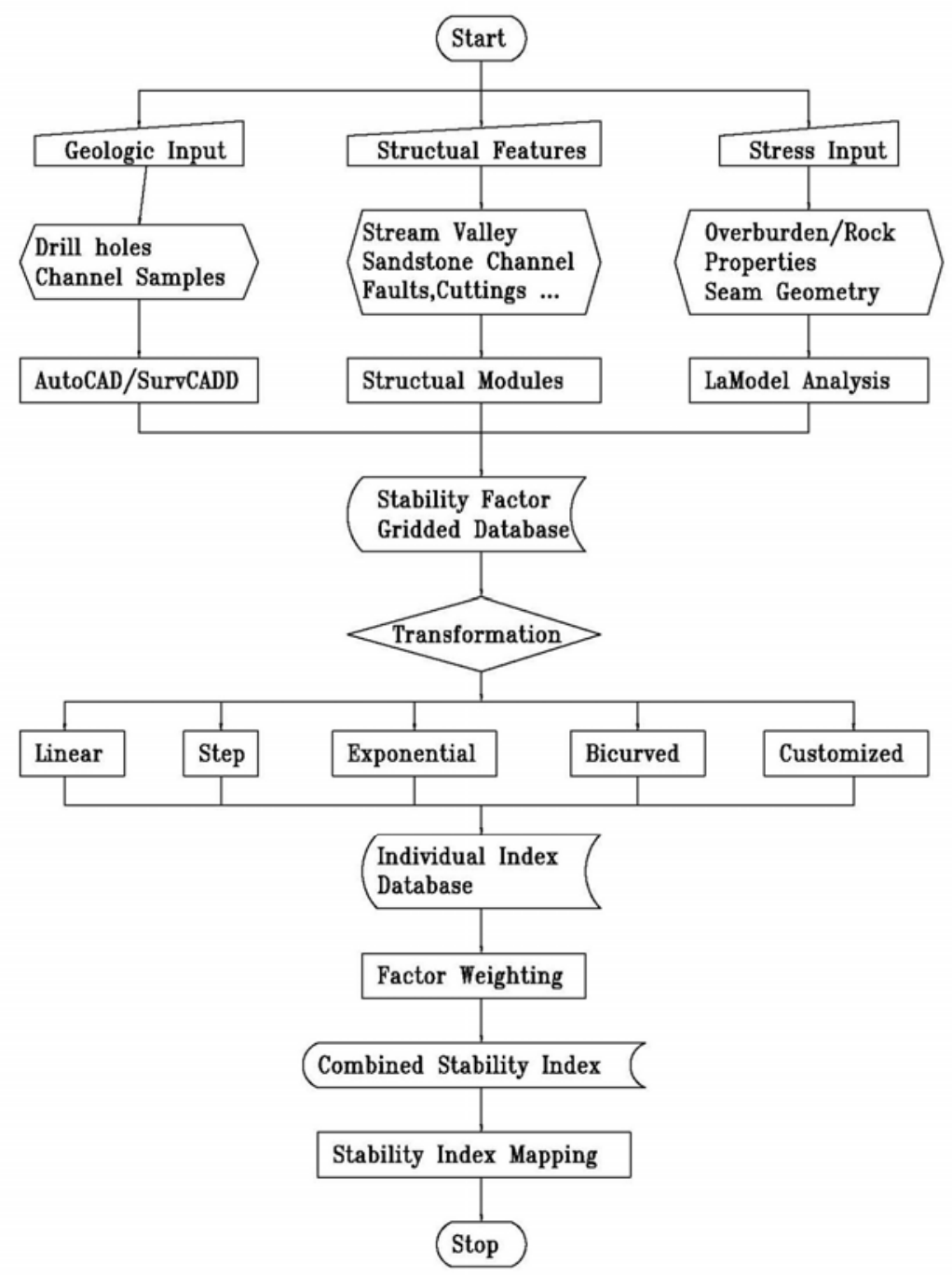

Figure 3.2. Typical data flow chart of stability mapping analysis 


\subsection{Requirements for Input Data}

In the design of the integrated stability mapping system, it is assumed that the necessary geologic and structural data are available in some AutoCAD compatible format such as points, lines, polylines, and/or SurvCADD grid files. In despite of these very different graphical representations in CAD system, the main input parameters of the influence factors can be divided into three categories: geologic properties, structural features and stress effects.

For the geologic part of the input, the standard SurvCADD program provides all of the basic geologic mapping capabilities that the final stability mapping program will need. With SurvCADD, the basic geologic characteristics such as layer thickness, strength, chemical composition, etc. are input from a variety of sources such as surface drill holes, underground samples or observations. Of course, this geologic information must be available to the user, and the quality of the calculated stability factor is directly dependent on the quality and density of the input geologic information. For a broad initial stability index calculation over the entire mine, the data from surface drillholes may be sufficient (Riefenberg, 1994). Once the mine is operational, the original drillhole information can be supplemented with underground boreholes and observations for a more detailed, more accurate and denser stability calculation. Present research into collecting continuous geologic information from the roof bolting machine (Finfinger et al., 2000) shows a lot of promise for providing extremely dense geologic information for input to the proposed stability mapping program. Irregardless of the source, once the user has acquired the necessary/available geologic data, the pertinent information can then be gridded. 
Since the mapping system will directly extract all input data from AutoCAD formatted files, all the geologic contours and structure features such as stream valleys, sandstone channels, etc., have to be represented as native entities inside the AutoCAD maps. These entities may be points, lines or polylines depend on the nature of the features and the preference of the data importer. However, the integrity and purity of these representations have to be examined before applying automatic gridding. For instance, when applying gridding on the topographic contour lines, these contours are required to be polylines with proper elevations and without intersections.

For the stress input, the output of a LaModel analysis is intended as the key input for geo-mechanical stress components of the system. Therefore, the original LaModel calculation is reconstructed as a individual module to be integrated into the mapping system. The initial LamPre program is a promising tool to prepare the input data for stress calculation. However, in order to speed up the preparation of the mine grids for LamPre in complex mine conditions, a number of enhancements have been completed and integrated into the stability mapping system, such as the capabilities that can automatically generate mine grid from underground mine pillar layout, and overburden grid from topography or overburden contours in AutoCAD mine maps.

\subsection{Stability Mapping Modules}

As stated early, a number of functional modules are assigned to accomplish different mapping tasks in the developed system. These modules can be classified into the following major groups by their designated goals: 


\subsubsection{Geology and Geo-Structural Gridding Modules}

These modules are developed to calculate the quantitative values for all critical geologic properties and structural features. Since their representations may take very different formats in mine maps, individual module will be designed to handle the full range of anticipated types of features. For example, the stability of a mine may be known to degrade under a stream valley (Stankus et al., 2001; Watts et al., 2000) within a certain horizontal distance from the stream at the bottom of the valley. In this case, the center line of the stream may be present on mine maps, and then the user can specify the critical horizontal distance of the stream valley effect. With this influence distance and the center line of the stream valley, a bounded area can be defined as an influence boundary for the stream valley. Then an area based gridding module will automatically check each grid element and assign an individual "stream" influence factor to the grid points within the critical area.

Generally speaking, geology properties and geologic structures naturally exist and have been recorded in some recognizable form through exploration surveys or field observations. When these data were transferred into CAD maps, their representation forms and data formats are diverse, since the data may from the various sources, mining engineers who perform the mapping have their own preferences, and different geology modeling techniques may be applied. Therefore, the geology oriented modules in the stability system should be generalized and applicable to common graphic representations of the geologic properties in the CAD system.

As any popular CAD system, the fundamental geometric objects representing geologic features in AutoCAD system can be abstracted as four types: points, linear 
objects, contour lines and closed areas. The points normally may be three dimensional survey data with $\mathrm{z}$ standing for the measured data while $\mathrm{x}$ and $\mathrm{y}$ are the locations of the measurement. Geology properties derived from spatial drill-hole logs are typically point type and the $\mathrm{z}$ values could be surface elevation, layer thickness, depth, chemical composition, etc. The linear objects refer to the lines or polylines in AutoCAD system and generally represent geologic structures that have linear characteristic such as factures, center lines of faults and stream valleys. Contours (3D polylines) are the most common methods used to graphically display various geologic properties of tabular seam deposition. Some typical examples are seam elevation, overburden depth, seam thickness and ash content of seam. Closed areas (closed polylines and polygons) are for special areas with closed boundaries, for instances outlines of stream valleys, ponds and rivers on the surface, and abandoned mine areas. For each type of abstract data structure, a respective module has been designed and implemented with appropriate algorithms in the stability mapping system.

\subsubsection{Stress Analysis Modules}

For integration of the stress value from LaModel into the stability mapping system, a number of interoperabilities and additional functionalities have been added to extend the use of the present LaModel philosophy. First, the capability of automatically generating the material (seam) and topographic grids for LaModel from mine map files of the mine plan and topography has been completed. With this capability, the stability program is capable of creating the LaModel input grids with minimal user input. Then, the capability of internally executing the LaModel stress calculation has been 
incorporated into the stability program. The original LaModel can calculate a number of outputs such as overburden stress, total vertical stress, seam displacement, etc. These original LaModel/MULSIM outputs can be selectively transformed to compatible grid file for use in stability system.

Although the initial LaModel calculation can produce comprehensive analysis results, the procedures from preparation of the input data with LamPre, calculation with LaModel to transformation of outputs can discourage users when only overburden stress is needed to be concerned in many cases. Therefore a separate module was developed to directly compute the overburden stress from the system by using the same algorithm as LaModel program.

In summary, to facilitate the LaModel stress analysis, a number of modules are designed in the stability mapping system including:

- Automatic seam grid generation

- Automatic topography grid generation

- LaModel calculation

- LaModel/MULSIM Output transformation

- Overburden stress calculation

\subsubsection{Horizontal Stress Module}

As discussed in section 2.3, both the orientation and magnitude of horizontal stress may impact the mine stability. The influence of the maximum horizontal stress direction on mine stability has been well documented, but the other important aspects of 
the horizontal stress, the magnitude and the magnitude variation, are not well understood. In the past and present, researchers have been trying to link the overburden depth and the increase in the magnitude of the horizontal stress by some empirical equations from statistical analysis. However the correlation is weak and how the magnitude affects the final roof stability locally and regionally remains uncertain and controversial.

A lot of studies have indicated that effect of horizontal stress orientation on mine entry stability depends on its angles to the orientations of the entries. An individual module is developed in order to reflect this influence in stability analysis. This module will examine the direction of entry development against the orientation of local horizontal stress, calculate the angles between them and assign appropriate influence index according to the magnitude of the angles

\subsubsection{Stability Factor Transformation}

Since the numerical values that are calculated for the various critical factors typically have different magnitude ranges and different influence weightings, they have to be converted to an individual stability factor with a consistent normalized value. An index range from 0 to 100 is chosen in the stability system, since it is consistent with the common connotation of the mine stability index, which suggests that the larger index value implies less stability of roof condition. A number of different "transformation" functions such as linear, bilinear, square, etc. have been built into the transformation modules. A user defined stability factor which may be a combination of the existing parameters is also derivable from the provided modules. Figure 3.3 shows a typical 
transformation relationship between the stability index and the distance of a grid point to a stream valley with a $50 \mathrm{ft}$ influence distance. In this example, the stability index of stream valley at any point is linearly proportional to the inversed distance from that point to the center line of the stream valley. When the distance is 0 , an index of 100 will be assigned to this point, while for points that are more than 50 feet, the lowest index is specified.

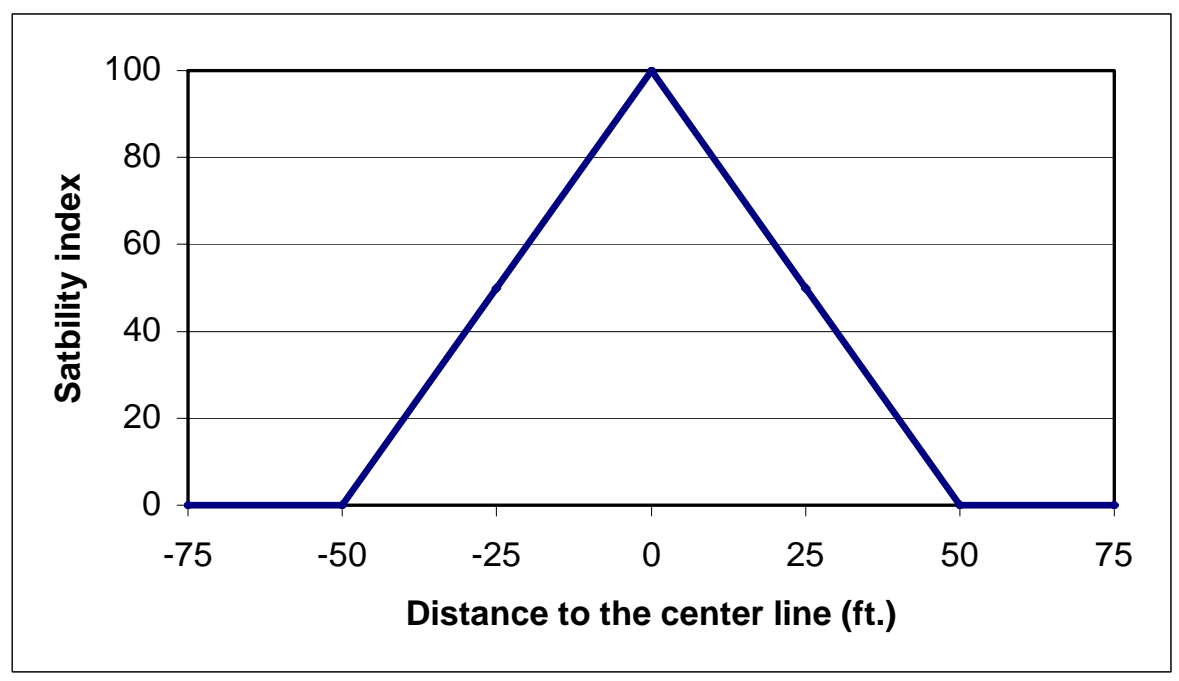

Figure 3.3. Linear inversed distance transformation

\subsubsection{Stability Index Modules}

Once all of the individual stability factors are determined for each of the critical parameters, the final stability index can be calculated as a combination of the individual factors. Typically, a standard weighted average summation approach is sufficient for most cases. The final stability index module provides a form for the user to input the individual factor grid files with their respective weights. These weights may be input as percentages or as relative integers. A final composite stability index is computed using 
these inputs. There are cases where a specific combination other than weighted average method is desired. In these occasions, the stability system provides a powerful grid utility module to accomplish the tasks (see 3.4.6).

A desirable representation of stability mapping is to illustrate the distribution of relative stability against the underground entry layouts. An index mapping module is designed to perform the function. The resultant drawing will be placed in a separate layer overlain on the mine map. Then one can clearly tell which segments of the entries may expect more unstable condition.

\subsubsection{Grid Utility Module}

The core data structure of the system is a rectangle grid, in which each element represents an area with a constant value of the properties. The outputs of all functional modules of the system are written into a consistent grid format. A grid utility module is designed to provide comprehensive manipulation of the grid values and grid files. A number of arithmetical operations within single grid or between two grids are included in the module such as addition, subtraction, multiply, division, etc. Using this module one is able to flexibly construct complex combinations of a stability factor (index) from multiple influence factors (indices).

Another role of grid utility module is to provide as a plotting routine for the output of stability mapping system. Output grid file can be previewed and/or plotted in specified AutoCAD layer with various different drawing settings. The drawings then can be managed by using any standard AutoCAD commands to meet user's special needs. 


\subsection{Summary}

In summary, the stability mapping system provides a comprehensive solution for the current mine stability mapping practices by integrating a variety of functional modules into one packages. These modules help/facilitate every facet of a typical stability analysis from data collection, automatic influence factor calculation and transformation, intermediate data managing, to final stability index rendering. In the design of the system, the mine stability factors are categorized into three groups, namely geology properties, structural features and stresses. For a specific mine, a set of critical factors needs to be determined at first. Then for each of the factor, an appropriate module may be used to compute the numerical values over the area of interest. After these factors are quantified into the system database, the transformation and other stability modules then can be used to integrate these factors into a composite stability index. The next chapter will present a detailed description of the algorithm and implementation of the key modules. 


\section{Chapter 4. Implementation of the Stability Mapping System}

\subsection{General}

The systematic analysis of the geologic and geo-mechanical factors on mine stability and the discussion of the general stability/hazard mapping procedures in previous chapters demonstrated a demand for a fast and accurate software solution for an integrated stability mapping system. Combined with the most advanced numerical method and computer-aided design technology, a comprehensive stability mapping system was initialized and developed. The application scopes and the requirements for input data and functional modules were also described in previous chapter. To create the stability mapping system and make it available for practicing mining engineers, a number of designated functional and auxiliary modules were programmed and integrated into a comprehensive stability program. This system was built into the AutoCAD/SurvCADD environment with an independent menu system, graphic user interface, fast numerical calculation and visual solution output.

This chapter illustrates the implementation of the integrated stability mapping system in more detail including algorithms, data/program flows, and input/outputs of then system's key components.

\subsection{Fundamental Data Structure of the System}

The fundamental data structure of the stability mapping system is a rectangular grid where each grid element represents a small mine area with consistent characteristic. This data structure underlies the entire system and serves as a bridge between all independent module components. A corresponding grid file is used to store immediate 
data and analysis results on the disk. In order to fully utilize the advanced SurvCADD geologic modules and existing SurvCADD formatted data resources, the grid file was designated to be compatible with SurvCADD grid file. A detailed description of the grid file and its relationship with SurvCADD can be seen in appendix A.

The grid structure is delineated as a three dimensional mesh in which the $\mathrm{z}$ values express the value of the specific stability influence factor in the element locations. The dimension of grid needs to cover the whole area of the interest. All the critical influence factors to be considered in the stability analysis are gridded using appropriate modules. The grid dimension for each factor is not necessary the same, but they have to be able to cover the entire area of the interest.

The grid is defined by specifying the grid location and grid geometry. The grid location is the coordinate of the grid origin, the low-left corner of the mesh. The grid geometric parameters consist of the element size (width) and the number of elements in both the $\mathrm{x}$ and $\mathrm{y}$ directions.

All the parameters can be typed into the grid definition form or interactively specified inside the AutoCAD drawing area using mouse. It is also possible to import the grid definition from previously existing grid file. The detailed grid input form can be found in the appendix B.2 of this dissertation.

\subsection{Geology Oriented Modules}

For the evaluation of mine stability, geological data have typically been the essential parameters to be investigated. Since any one of a broad ranges of geologic properties may impact the mining conditions, it is desirable to divide them into different 
categories. In the stability mapping system, the geologic properties and structural features were used as two distinct geological data types.

The geologic properties refer to the traditional geological inputs such as seam thickness, overburden depth, seam compositions, etc. These type of data usually were imported and interpreted from drill hole exploration and underground channel samples by mine geologists. The process creating a grid of a critical geologic factor can be done by using the proper SurvCADD module if accessible. The stability mapping system also provides the basic modules to allow the geologic data to be evaluated as long as the sufficient spatial data or contours for this geologic property are available.

On the other hand, the structural features include abnormal geological or geographical features underground or on the surface. This includes such features as stream valleys, sandstone channels, faults, cutters, etc. In general, their influences on the mine stability are subject to limited by distance. That means that a structural feature only has impact over a certain distance around or inside its outlining area. Although there are a variety of structural features, we can abstract them into two basic data types depending on their representations in the mine maps. The first one is one-dimensional linear features such as a fault or stream. The linear structural feature is usually represented by its center line on mine maps. Its influence on mine stability is typically considered as linearly proportional to the distance from the center. The second type of structural features is an area-based geological structure, which is characterized by a polygonal bounded area where insides or outsides of the boundary the structural feature affect the stability. 
In general, for geology oriented influence factors, four distinct gridding modules were developed to evaluate different input data types, namely points, linear structures, contour lines and special areas.

\subsubsection{Gridding Module for Spatial Points}

Spatial point data generally came from exploration drill holes and field investigations. It can be stored in a plain text file or as point objects in mine maps. It represents the magnitude $(\mathrm{Z})$ of a property at some available locations $(\mathrm{X}, \mathrm{Y})$. These three dimensional data are used to calculate the property's value for every grid element by using interpolation approaches. Figure 4.1 shows the detail flowchart of the solution.

The overall process in calculating property values at grid points is straightforward. First, point data locations (map layers or external files) and control settings (minimum number of points used in interpolation, maximum search radius) have to be input. The point data then will be loaded from the specified location. For each element location in the grid mesh, an incremental circular search will be conducted, until enough nearest neighbor points are found or the maximum search radius is reached. The selected interpolation method is then used to calculate the value for this element. Two basic interpolation methods have been implemented in the system, namely simple average method and inverse distance method.

- The simple average method: In this simplest method, the grid value at a particular point is the simple average value of the nearest neighbors (see Equation 4.1). 


$$
g_{i}=\frac{\sum_{j=1}^{n} X_{j}}{n}
$$

Where: $\mathrm{g}_{\mathrm{i}}$ is the estimated value of grid point $\mathrm{i}$;

$\mathrm{n}$ is the number of neighbor points;

$\mathrm{X}_{\mathrm{j}}$ is the value of neighbor points.

- Inverse distance method: This approach estimates the element value at a point by weighting the influence of nearby data the most and more distant data the least. Each neighboring value is weighted inversely proportional to its distance. This can be described mathematically by Equation- 4.2:

$$
g_{i}=\frac{\sum_{i=1}^{n}\left[\frac{X_{i}}{d_{i}^{p}}\right]}{\sum_{i=1}^{n} \frac{1}{d_{i}^{p}}}
$$

Where: $\mathrm{g}_{\mathrm{i}}$ is the estimated value at the node location;

$\mathrm{d}_{\mathrm{i}}$ is the distance between the interpolated point and the sample points; $\mathrm{p}$ is the power to which the distance is raised (usually $\mathrm{p}=2$ ) 


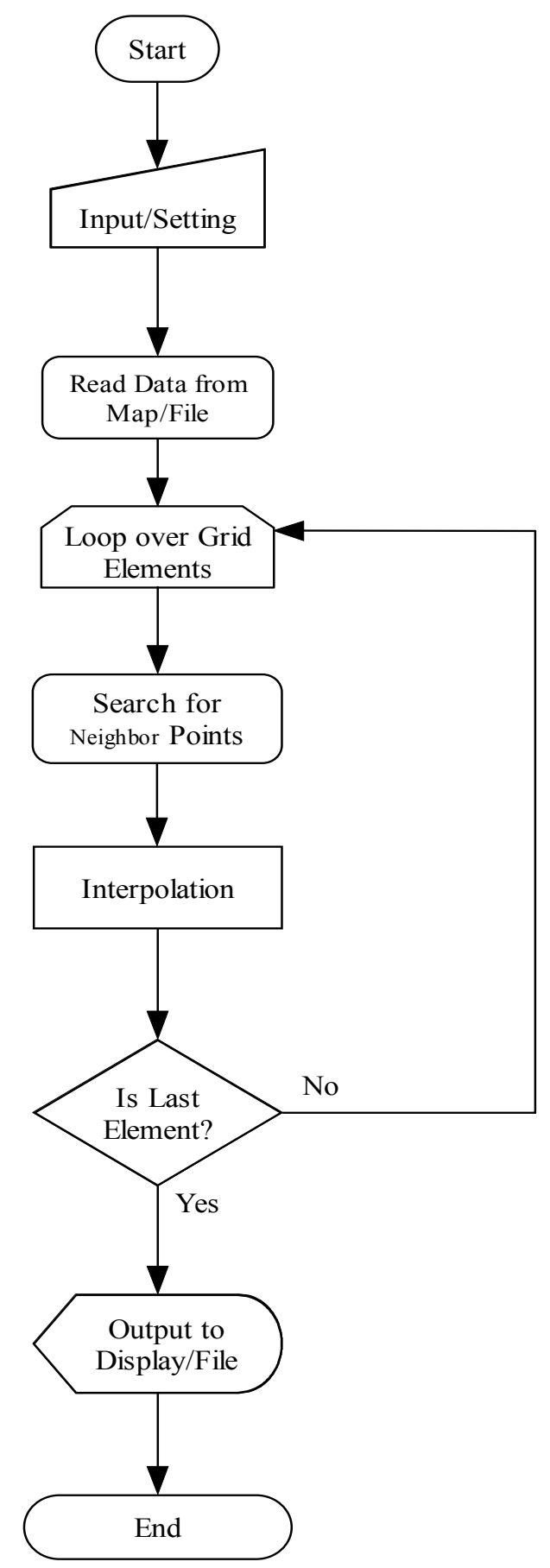

Figure 4.1 Details of the spatial point module 


\subsubsection{Gridding Module for Contours}

One of the most common representations of geological properties on mine map is the isopach maps where the $3 \mathrm{D}$ contour lines were drawn over the mine area to show the distribution of geologic properties. In order to extract the geologic data from these available contour lines, a contour module was developed in the stability mapping system. This module takes contour lines in specified layers as input. The grid values are calculated using the nearest points on the contours. The process of calculation is outlined in Figure 4.2.

In this module, after the contour layers are specified, all contour lines in the layers are filtered against the extent of the study area. Only relevant contour objects will involve the calculation of the grid values. For each grid element, the nearest point on each contour line is calculated. These "near" points are then sorted by distance to the grid elements. The first two points with different orientation or elevation are used to calculate the grid value by using the Inversed Distance Method (IDM) described in the last section.

In fact, when one wants to calculate a grid values from geologic contours, both point and contour based modules can be applied. In the process of point based module, when contour line objects are read from the specified layers, an internal implicit discretization process will divide the contours into discontinuous points with the interval size of a grid element. These points are then used as spatial point data to calculate the grid value as usual. 


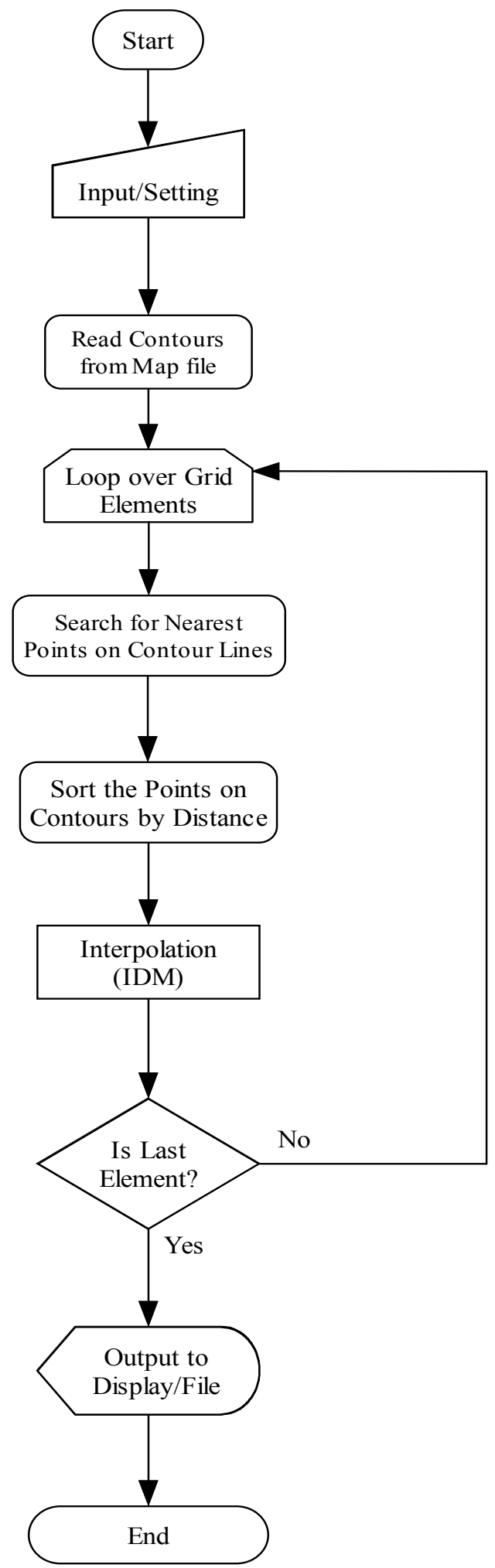

Figure 4.2 Details of the contour gridding module 


\subsubsection{Gridding Module for Linear Features}

The gridding module for linear feature is to calculate the influence factor for geological structure features with a linear representation on the mine map. These features are assumed to have an impact that is proportional to the distance between the feature and the specific grid location. Generally, the linear features are drawn as a lines or/and polylines in specific layers on the mine map. The module takes the linear objects and their user specified influence distance as input. Only the grid values of the elements inside the influence range will be updated.

The basic flow chart of this module can be seen in Figure 4.3. At first, the linear structural objects are read and the grid values are initialized to zero. Then for each line/polyline object, a boundary is constructed around the influence range. By using this boundary, all grid cells possibly affected by the object are defined. For each element, the distance $\left(\mathrm{d}_{\mathrm{i}}\right)$ to the structure is calculated and compared with the input influence distance $\left(\mathrm{D}_{\mathrm{i}}\right)$. If the distance is less than the influence distance, the influence factor is calculated with an inversed linear interpolation. If the influence "accumulation" is selected, the influence factor in end cell will be cumulative (see Equation 4.3, 4.4).

With accumulating influence:

$$
\text { Influence factor }= \begin{cases}0 & \text { if } d_{i}>D_{i} \\ \sum \frac{100}{D_{i}}\left(D_{i}-d_{i}\right) & \text { if } d_{i} \leq D_{i}\end{cases}
$$

Without accumulating influence:

$$
\text { Influence factor }= \begin{cases}0 & \text { if } d_{i}>D_{i} \\ \operatorname{Max}\left\{\frac{100}{D_{i}}\left(D_{i}-d_{i}\right)\right\} & \text { if } d_{i} \leq D_{i}\end{cases}
$$




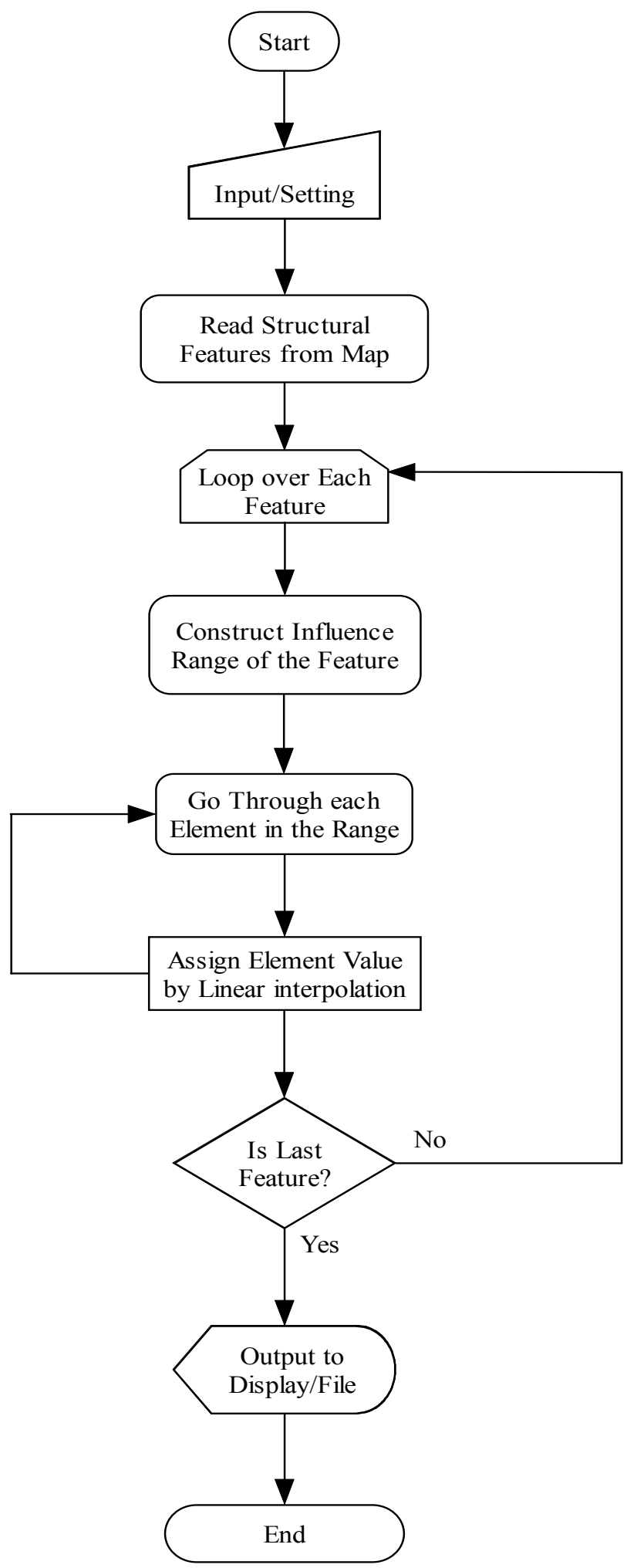

Figure 4.3 Details of linear feature module 


\subsubsection{Gridding Module for Area-Based Features}

Another major category of structural feature is the area-based geological structures. This type of feature would be represented as closed polygons/polylines on the mine maps. Some typical area-based structural features are stream valleys, sandstone channels, etc. The stability influence of the area-based features is determined by checking if the grid cell location is inside the area of the features or not. In general, if a grid element is located inside the outline area, the grid value will be assigned to 100 , otherwise 0 .

There are two important steps in the flow chart for the area-based module (see Figure 4.5). The first step is to create the influence boundaries for the input features. The influence boundaries can be built from either center lines or closed outlines of the features with a specified influence distance. The second important step is to identify if a grid element is inside the boundaries or not. Two different algorithms were implemented in the module to accomplish this second task namely the middle point method and the area-based method.

The middle point method uses the middle point of the grid element as the critical element point. If this point is inside one of the boundaries, we assume the whole element is inside the boundary. Determining if a point is inside an arbitrary polygon (convex or non-convex) is a typical computational geometry problem. There are two popular solutions to solve this problem: computing "winding" numbers and counting ray crossing.

The "Winding" number algorithm is a mathematically pleasing method which calculates the total angular turn for all lines that runs from the query point, q, to each 
vertex point, $\mathrm{p}$, on the polygon. If the point $\mathrm{q}$ is inside polygon, the total angular turn would be a full circle $(2 \pi)$, whereas if $\mathrm{q}$ is not inside the polygon, the winding number would be exactly zero. Although the winding number algorithm is appealing, its dependence on trigonometric floating-point computations makes it significantly slower than the ray crossing algorithm.

The theory of the ray crossing algorithm implemented in the stability module is very simple. An imaginary ray is draw from query point $\mathrm{q}$ in an arbitrary direction and the number of intersects of the ray with the polygon $\mathrm{P}$ is counted. The point $\mathrm{q}$ is inside the polygon if the number of crossings is odd and it is out of polygon $\mathrm{P}$ if the number of crossings is even. A special case for this problem is how to handle the situation when the point $\mathrm{q}$ is a vertex or on the edge of polygon. In this module, for the case of $\mathrm{q}$ on the polygon we consider it is inside the polygon.

The Area-based method examines the overlap area between the feature boundary and the element rectangular. The element is considered inside the polygon when the overlapped area is larger than a specified percentage (typically 50\%) of the element area. Computing the overlapped area involves solving another computational geometry problem: the intersection of two polygons. The intersection polygon is defined by clipping each grid element polygon with the geological feature boundaries.

In general, a polygonal boundary would be clipped against each edge of the element polygon one at a time. The cell/edge intersections, if any, kept after clipping against one cell edge are saved for clipping against the remaining edges. 
The clipping algorithm applied in the module is similar to the SutherlandHodgman polygon clipping algorithm:

Within this algorithm, each vertex of the polygon is examined against the clipping plane defined by the line segment of the cell polygon to check if the vertex inside or outside the segment plane. The term inside means that the clipping plane's normal vector points towards the point (Figure 4.4 (a)), as defined by equation (4.5):

$$
\left(\stackrel{1}{p}-\stackrel{1}{p}_{0}\right) \cdot \stackrel{1}{n} \geq 0
$$

Where $\stackrel{1}{p}$ is the point in query;

${\stackrel{\mathrm{I}}{p_{0}}}_{0}$ is a point on the plane;

$\stackrel{1}{n}$ is normal to the plane;

The outside means not inside and the intersection is the intersection point of the edge in question with the clipping plane. The algorithm can be explained simply:

For each edge of the polygon, check both nodal values, $s$ and $p$. If the point values are:

- Inside-inside, append the second node, $\mathrm{p}$.

- Inside-outside, compute and append the intersection, $i$ of edge sp with the clipping plane.

- Outside-outside, no operation.

- Outside-inside, compute and append the intersection $i$ of edge sp with the clipping plane, then append the second node $\mathrm{p}$.

The resultant ordered list of nodes forms the clipped polygon. A simple example of this clipping algorithm is shown in Figure 4.4 (b). 


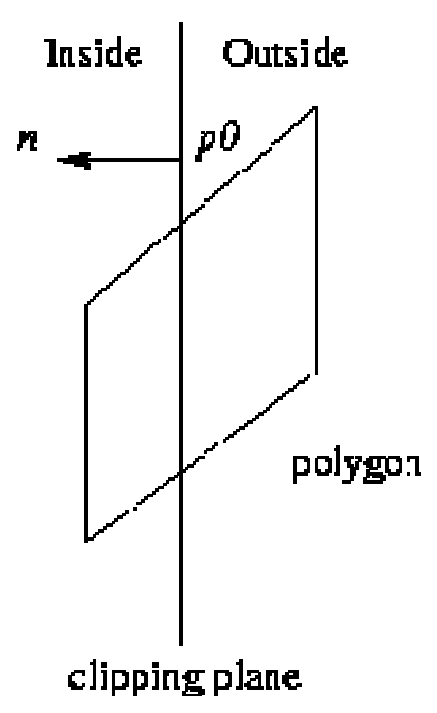

(a)
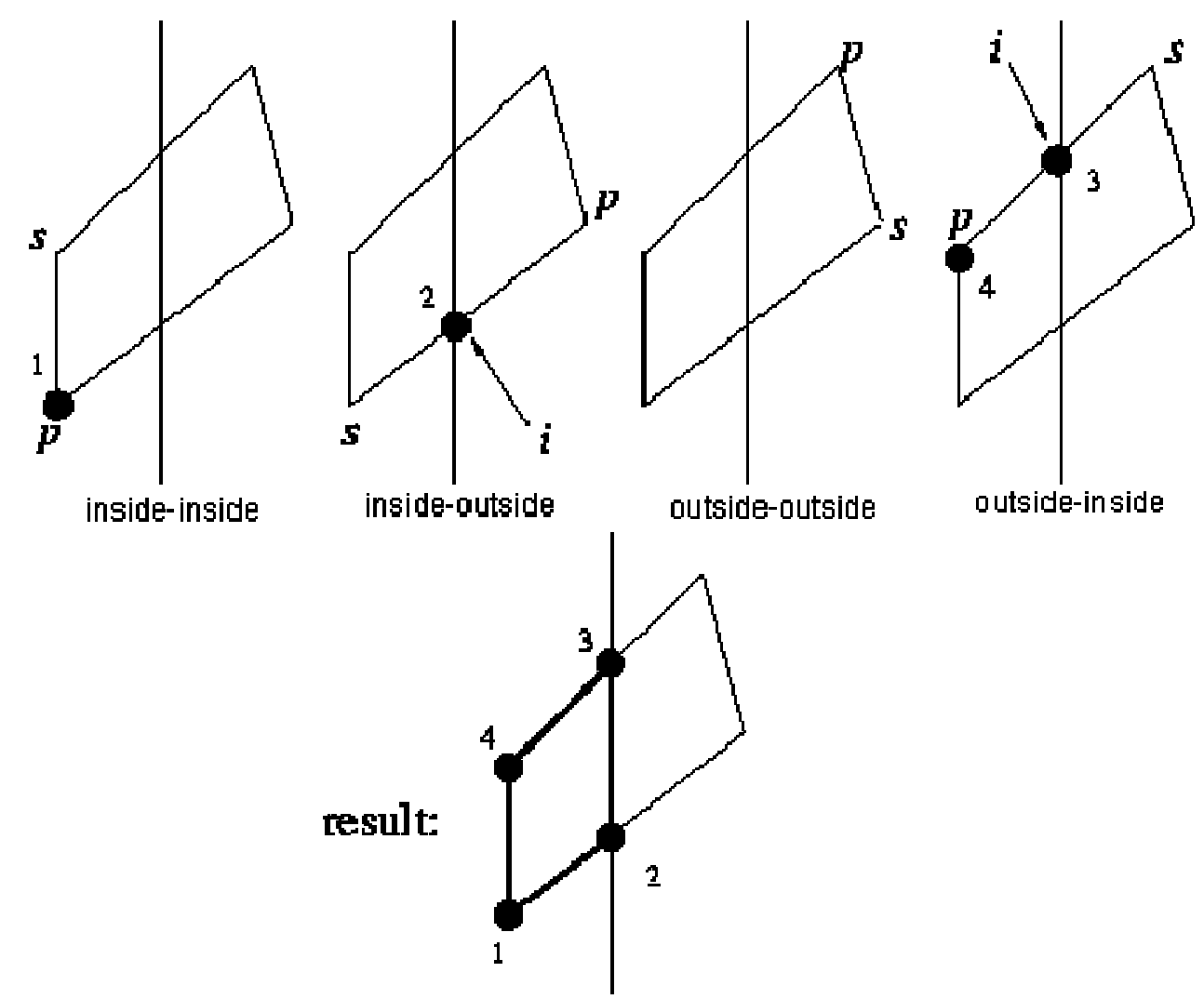

(b)

Figure 4.4 Polygon clipping algorithm 


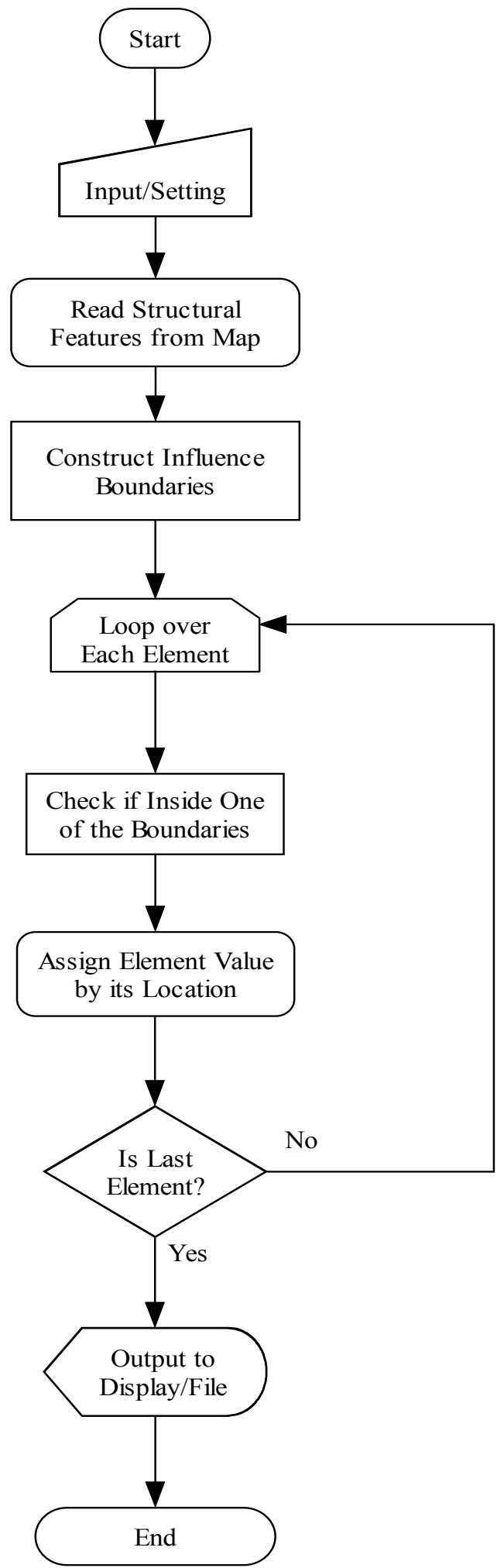

Figure 4.5 Details of gridding module for area-based feature 


\subsection{Stress Oriented Modules}

As stated in the previous chapter, the LaModel stress calculations are incorporated into the stability system. In addition to the ability of easily converting the typical LaModel results into the system grid format, a number of utility functions have been built in the stability mapping system in order to improve the accuracy and utility of the LaModel stress analysis, and to simplify the process of the data input of LaModel. These functional modules include:

- Automatic overburden/topography grid generation

- Automatic seam grid generation

- Overburden stress calculation

Automatic overburden/topography grid generation module is to generate an input file for LaModel. This file contains the overburden thickness over the study area, and is used by LaModel to calculate the initial vertical stresses. The module takes contours of overburden thickness or topography elevation as input data, and applies the same logic and algorithms as the gridding module for contour lines discussed in section 4.3.2 to calculate the thickness values. The result of this module is a formatted text file, which is required by LaModel application as a topographic input file.

Similarly, the automatic seam grid generation module is to create the proper seam grid definition for LaModel, in which each grid element has a specified material property. This module follows the same strategy and algorithms as the area-based structural module. Basically, it takes in-seam pillar layouts (closed polylines on the map) as input, checks over all grid elements against the pillars. If the grid element is located inside a pillar, grid value is assigned to "A", which means a coal element, otherwise "1", which 
implies an opening element. An example can be seen in Figure 2.13. The resultant seam grid accurately represents the on-site pillar system, and improves the accuracy of the final stress calculation.

In following section, the overburden stress module and the horizontal stress module will be discussed in more details.

\subsubsection{Overburden Stress Module}

In evaluating geo-mechanic influences on mine stability, the overburden stress is a factor that is often analyzed. In particular, when one wants to predict the mine stability for a new mine in steep or mountainous topography where no seam extraction has been conducted, the overburden stress would probably be one of the dominate factors impacting the future mine stability. Therefore an overburden stress calculation module has been implemented in the stability mapping system. This module numerically implements the same laminated overburden model as in LaModel.

Basically, the overburden stress algorithm simulates the variable topography as a collection of normal stresses on a horizontal datum plane at the elevation of the average overburden with the magnitude of the normal loading being proportional to the elevation difference between the overburden of each point and the average overburden elevation. The pseudo-stresses on the datum are used to determine the pseudo-displacements of the datum by using a Gauss-Siedel Successive Over-Relaxation (SOR) solution to the finite difference equation (4.6). Then the overburden stresses of the seam level are calculated by numerically integrating the pseudo-displacements on overburden grid using a derived 
influence function (4.8) (Heasley, 1998). These two fundamental equations for a laminated overburden model had been derived by Heasley in his dissertation in detail (Heasley, 1998).

$$
\frac{\partial^{2} s}{\partial x^{2}}+\frac{\partial^{2} s}{\partial y^{2}}=\frac{2}{\lambda E} \sigma_{i}
$$

Where: $\mathrm{s}$ is the convergence in the seam

$\sigma_{\mathrm{i}}$ is the induced stress in the overburden

$\lambda$ is a constant property defined by strata lamination thickness $(\mathrm{t})$ and

Poisson's Ratio (v) of the overburden laminations.

$$
\begin{gathered}
\lambda=\frac{t}{\sqrt{12\left(1-v^{2}\right)}} \\
F=S_{l} \frac{E}{8 \pi \lambda z^{2}}\left(\frac{x^{2}+y^{2}}{4 \lambda|z|}-1\right) e^{-\frac{x^{2}+y^{2}}{4 \lambda|z|}}
\end{gathered}
$$

Where: $\mathrm{F}$ is vertical stress in a laminated overburden

$S_{l}$ is local seam displacement

The figure 4.6 shows the flow chart of the overburden stress calculation implemented in the stability system. In this module, three groups of data are required as input: a seam grid for the stress, which defines the dimension of the area of interest and storing the solution; a surface grid, which contains the overburden thickness or surface elevation; and overburden properties and control parameters (including the rock mass properties, lamination thickness of overburden, and convergence criteria). 


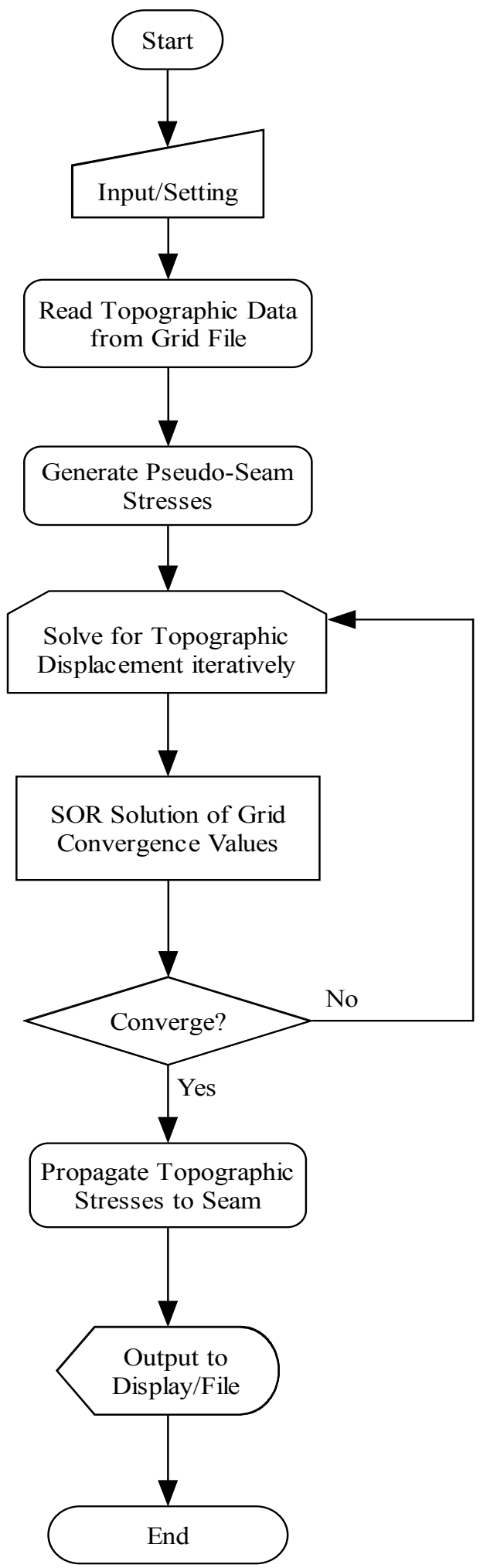

Figure 4.6 Details of the overburden stress calculation 


\subsubsection{Horizontal Stress Module}

The horizontal stress module is developed to incorporate the influence of horizontal stress orientation into stability mapping. Typically, horizontal stress fields are biaxial with a maximum and a minimum horizontal stress. It has been well documented that in the U. S. coal fields, the maximum horizontal stress is generally 2-3 times greater than the minimum horizontal stress (Mark and Much, 1994; Keim, 1999). Consequently, the entries orientated perpendicular or nearly perpendicular to the maximum horizontal stress are subject to more stress-induced damage than entries orientated parallel to the maximum horizontal stress (Stankus, 1999; Gadde and Peng, 2004).

To determine the influence of the horizontal stress field on the mine stability map, the horizontal stress module computes an influence factor for the underground entry system by applying the Equation 4.9 as below:

Influence factor $=100 \times \sin (\theta)$

Where: $\theta$ is the angle between the center line projection of the entry and the orientation of the maximum horizontal stress.

Key inputs for the module are the projections of the mining entry system, entry width and the orientation of the maximum horizontal stress. The flowchart of the module is shown in Figure 4.7. After the entry center lines are read in, the module goes through each entry projection, calculates the angle between the projection and the maximum horizontal stress, and determines the influence factor from equation (4.4). Then all grid elements impacted by the projection are assigned this value. The process terminates until all entry projections have been examined. 


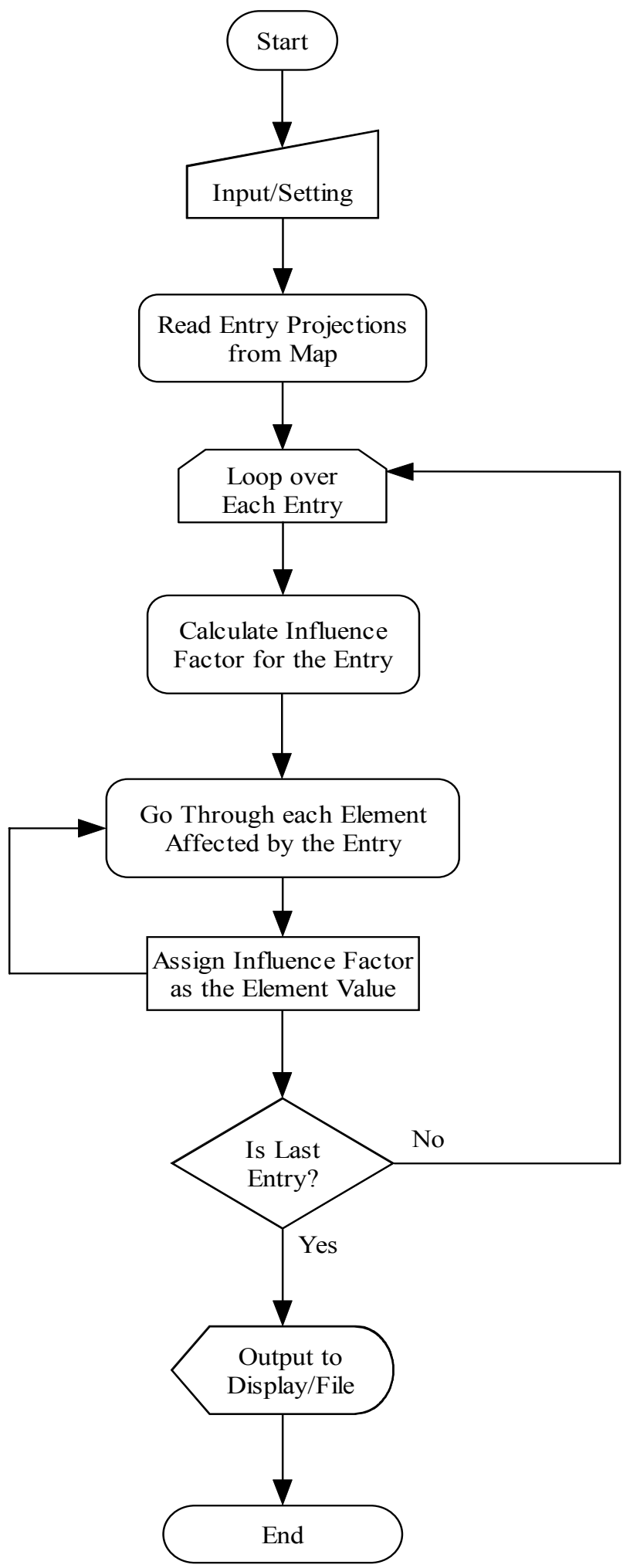

Figure 4.7 Details of the horizontal stress module 


\subsection{Index Mapping Modules}

The previous sections discussed the principle modules for calculating the numerical values for a variety of mine stability factors: geologic properties, structural features and geo-mechanics factors. It was shown that the numerical values of a critical influence factor can generally be computed directly, or indirectly, from its graphical representation on the mine map as soon as the required input data and parameters can be defined. Once the quantitative values for these critical factors are calculated, the stability index mapping modules described in this section are used to calculate the final stability index. The functions of these modules include influence factor transformation, integration and mapping.

\subsubsection{Stability Factor Transformation}

Once all of the critical influence factors have been input, gridded and saved in the database, the major effort in data input for the stability mapping system is complete and the core grid database has been created. The next step is to calculate individual stability indices by applying the appropriate transformation function to each factor. As we have noticed, the critical influence factors are representing the various properties in term of geology and mechanics. Therefore, they will have very diverse value ranges and varying implications for mine stability. For instances, the thickness factor of a specific layer in the overburden may spread from 0 to $50 \mathrm{ft}$, while the magnitude of the overburden stress factor for the same area may lay between 1000 and 2000 psi. On the other hand, for a geologic factor such as sandstone channel, a higher thickness may imply a weak mine 
condition, whereas for another factor such as the interval between the sandstone layer and seam level, a larger thickness might contribute to a more stable condition.

In order to eventually combine all of the individual critical factors into one overall stability index, each of the individual factors has to first be normalized and/or inverted into the same numeric range by using an appropriate transformation function. The transformation functions presently included in the stability system are Linear, Step, Exponential, and User-Defined, and the default numerical range for the individual and overall indices in the stability mapping system is 0 to 100 , with larger values typically representing less stable conditions.

- Linear transformation

Linear transformation is a typical method to normalize the individual factor to the standard (0-100) index range. There are two variation of linear transformation: normal and inverse. For a normal linear transformation, the numerical value of an influence factor is linearly mapped to the index range. The transformation is defined mathematically by equation 4.10 :

Individual stability index $= \begin{cases}0 & \text { if } d_{i}<D_{l} \\ 100 \times\left(\frac{d_{i}-D_{l}}{D_{u}-D_{l}}\right) & \text { if } D_{l}<d_{i}<D_{u} \\ 100 & \text { if } d_{i}>D_{u}\end{cases}$

Where: $d_{i}$ is the numerical value to convert

$D_{l}$ is defined lower bound of the numerical values

$D_{u}$ is defined upper bound of the numerical values

For an inverse linear transformation, the numerical value of an influence factor is inverted and linearly mapped to the index range by Equation 4.11 : 
Individual stability index $= \begin{cases}100 & \text { if } d_{i}<D_{l} \\ 100 \times\left(\frac{D_{u}-d_{i}}{D_{u}-D_{l}}\right) & \text { if } D_{l}<d_{i}<D_{u} \\ 0 & \text { if } d_{i}>D_{u}\end{cases}$

The linear transformation methods are illustrated in Figure 4.8. In this figure, Min and Max are the minimum and maximum numerical values of the original influence factor respectively.

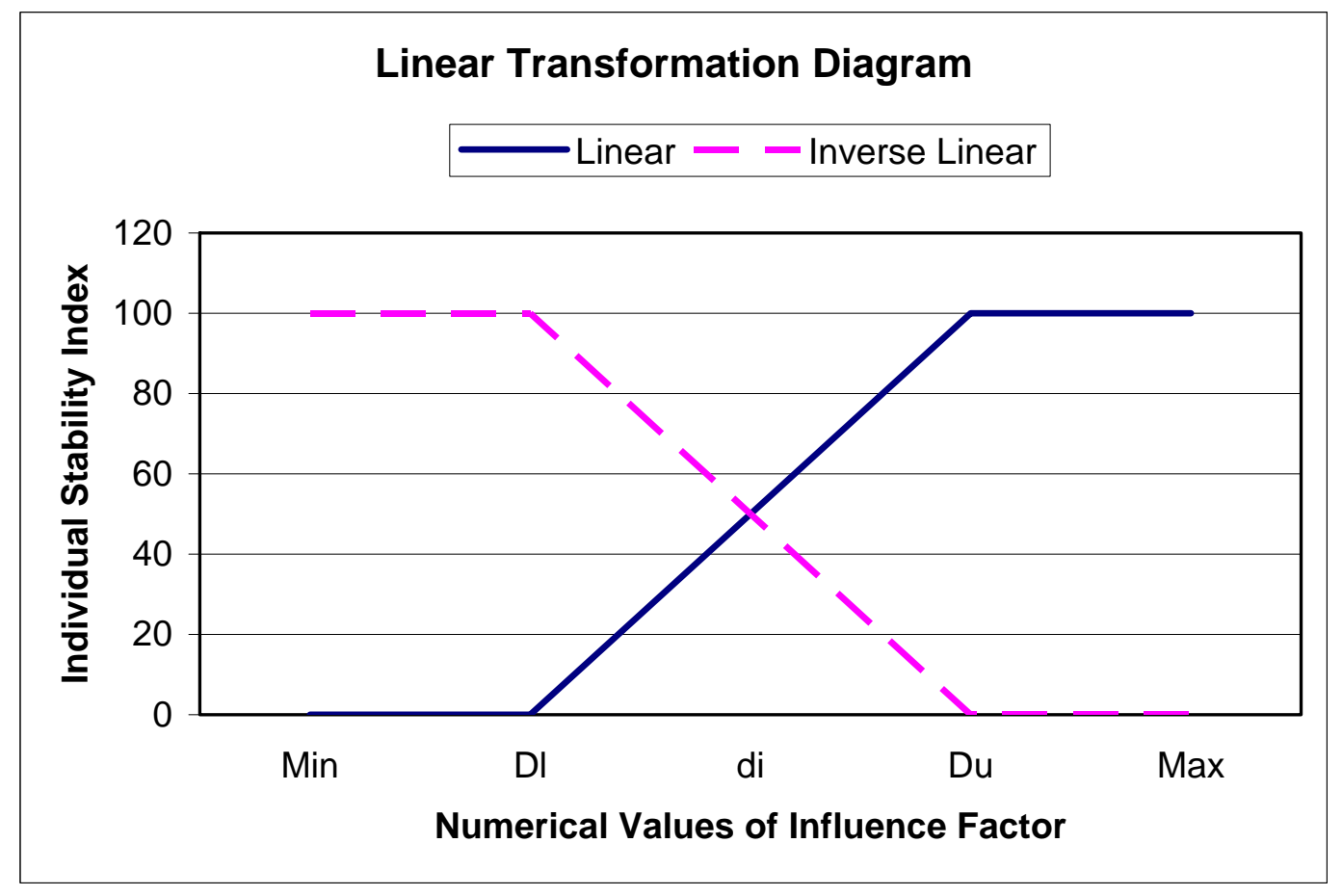

Figure 4.8 Linear transformation

As an example, assume that the interval thickness between a seam and a sandstone layer in the immediate roof has been gridded and that it ranges from 0.1 to 9.4 ft. It is desired to transform this interval thickness grid into an index grid that ranges from 0 to 100 . In this case, since a larger thickness implies a more stable condition with a smaller index value, the inverse linear transformation function should be used with the 
lower and upper bounds set at 0.1 and 9.4 respectively. This transformation would linearly map the 0.1 to 9.4 range into a 100 to 0 range (see Chapter 6 for complete example).

- Step transformation

A step transformation is fairly simple conversion method. It maps the numerical values of the influence factor into one of two stability index values, 0 or 100, depending on the implication of the actual property. Similar to the linear transformation, there are also two variations of this method: normal and inverse. In a normal step transformation, the individual stability index is defined by Equation 4.12:

$$
\text { Individual stability index }= \begin{cases}0 & \text { if } d_{i}<D_{c} \\ 100 & \text { if } d_{i} \geq D_{c}\end{cases}
$$

Where: $d_{i}$ is the numerical value to convert

$D_{c}$ is defined step point of the influence factor

In an inverse step transformation, the numerical value of an influence factor will be assigned by Equation 4.13:

$$
\text { Individual stability index }= \begin{cases}100 & \text { if } d_{i}<D_{c} \\ 0 & \text { if } d_{i} \geq D_{c}\end{cases}
$$

Take the example of the previous linear transformation of the interval thickness. It is could be that at this property it has been found that stability problems typically only occur when the thickness is less than $2 \mathrm{ft}$. In this case, the inverse step transformation function would be used with the step set at 2 . Therefore, all of the thickness values above 
2 would be transformed to an index of 0 and all of the values below 2 would be transformed to an index of 100.

- Exponential transformation

In reality, a critical influence factor may impact the mine stability in nonlinear manners. The exponential transformation was designed to accomplish this type of stability index transformation. In an exponential transformation, the stability index is defined as equation 4.14:

Individual stability index $= \begin{cases}0 & \text { if } d_{i}<D_{l} \\ 100 \times\left(\frac{d_{i}-D_{l}}{D_{u}-D_{l}}\right)^{p} & \text { if } D_{l}<d_{i}<D_{u} \\ 100 & \text { if } d_{i}>D_{u}\end{cases}$

Where: $d_{i}$ is the numerical value to convert

$D_{l}$ is defined lower bound of the numerical values

$D_{u}$ is defined upper bound of the numerical values

$p$ is exponential power

For an inverse exponential transformation, the numerical value of an influence factor is invert and mapped to the index range by equation 4.15 :

Individual stability index $= \begin{cases}100 & \text { if } d_{i}<D_{l} \\ 100 \times\left(\frac{D_{u}-d_{i}}{D_{u}-D_{l}}\right)^{p} & \text { if } D_{l}<d_{i}<D_{u} \\ 0 & \text { if } d_{i}>D_{u}\end{cases}$ 
Figure 4.9 shows the relationship between the transformed stability index and the numerical value of an influence factor with p equal to 2 and 3.

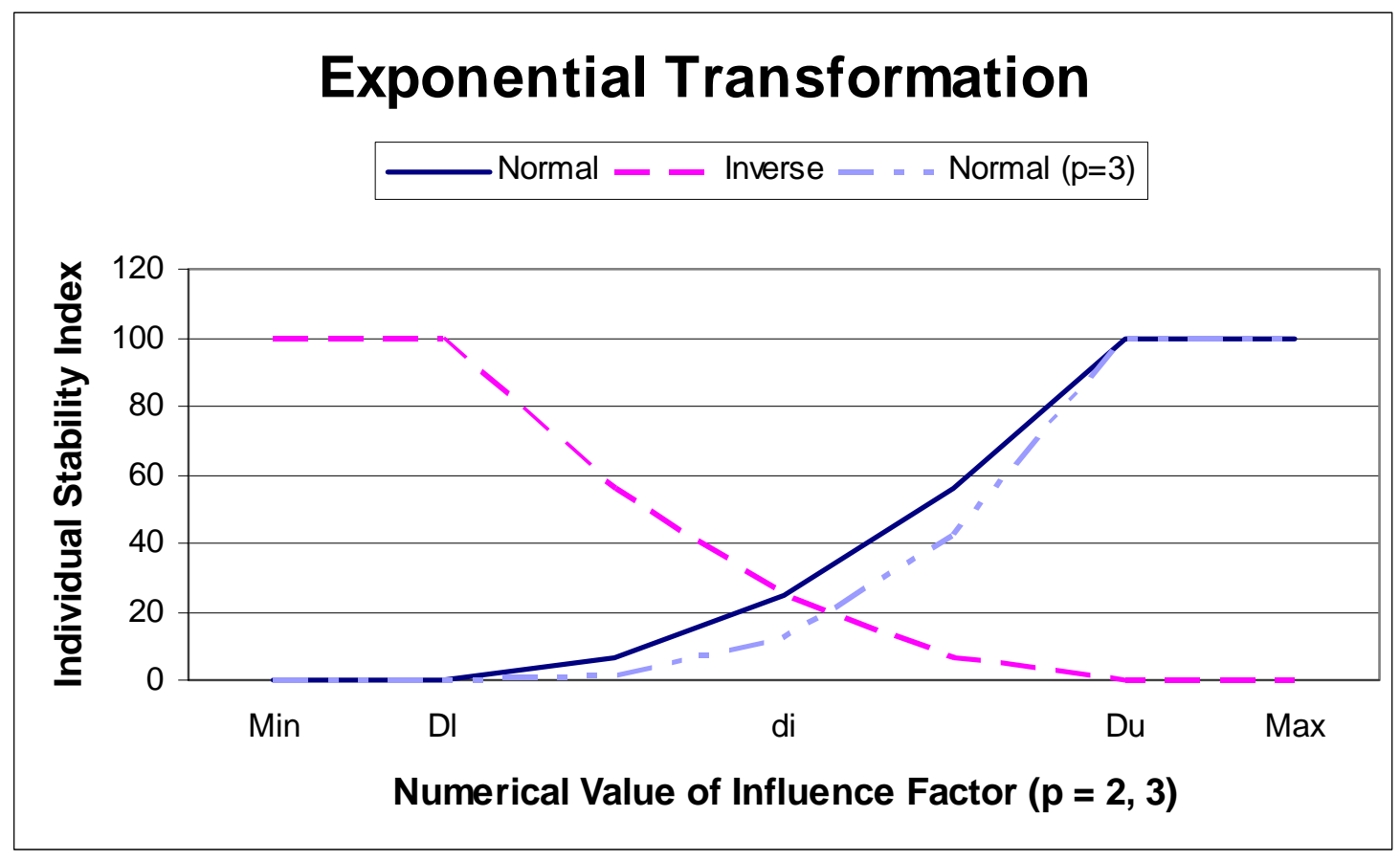

Figure 4.9 Exponential transformation

Form figure 4.9 , it can be seen that the different " $p$ " values will cause different transformation behaviors. For instance, in the normal transformation, with an increasing "p" value, the curve of transformation will grow slower than with a smaller "p" value. It means the numerical value will be converted to a smaller individual stability index when the "p" value increases. 
- User defined transformation

The three basic transformation methods discussed above have been built into the stability mapping system. These transformations are applicable to many situations that may be encountered. However, there are cases that mine engineers may have a sitespecific transformation method from their past experience, and it is not one of the three predefined methods. The stability mapping system has provided a multiple step approach for such a transformation by manipulating the influence factor grid with a number of numeric operations such as addition, subtraction, multiplication, division, square, square root and logarithm. These numeric operations allow mine engineers to develop their own transformation methods with limitless flexibility.

With the available transformation functions, there are numerous approaches that can be used to logically map a critical input factor into an appropriately scaled stability index. In general, selection of a proper transformation method depends on the nature of the influence factor and may require some on-site experiences and calibration for a specific site location.

The implementation of the stability index transformation module is straightforward. After the grid values are read from the influence factor grid file, the program goes through each element value, applies the selected transformation to the element until the values of all elements are processed. The resultant index grid file is created with the transformed grid value. Figure 4.10 shows the flowchart of the module. 


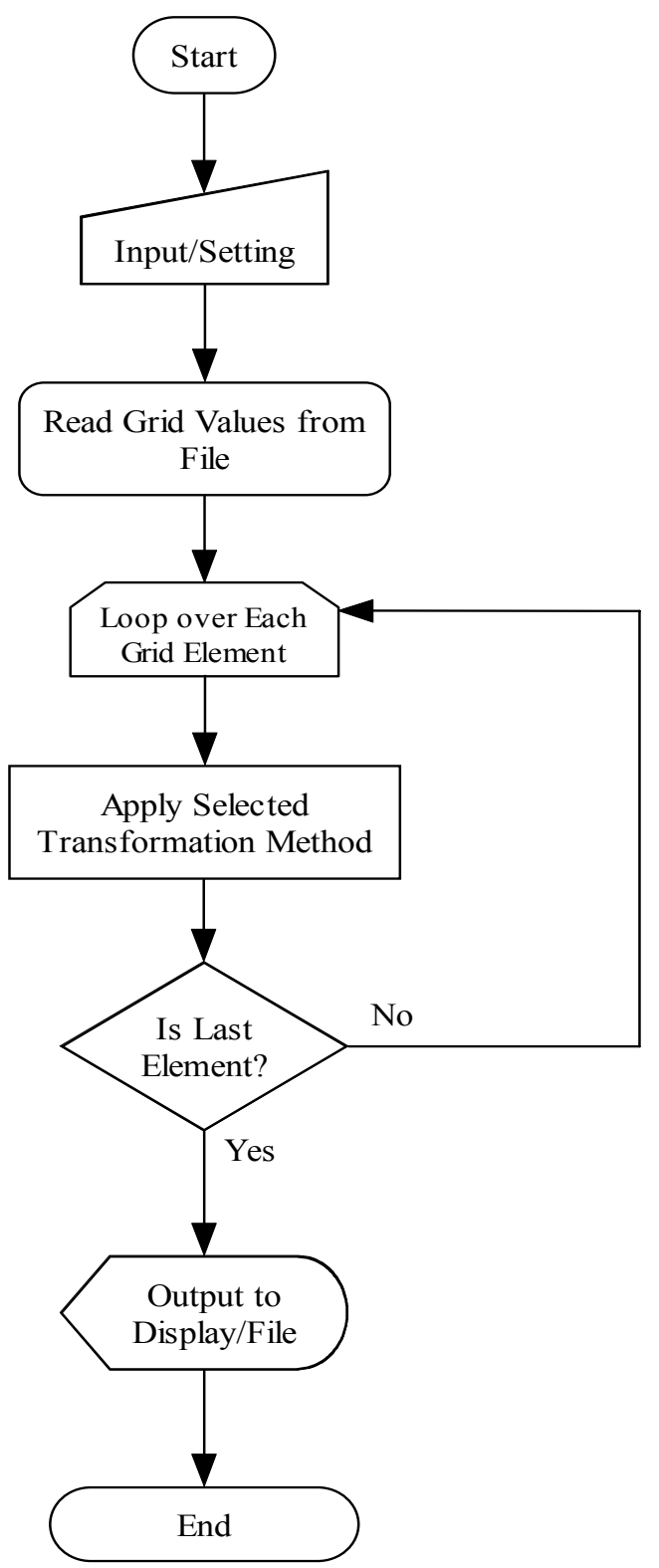

Figure 4.10 Details of transformation module 


\subsubsection{Final Stability Index Module}

Once all of the individual indices have been determined using the appropriate transformations, the next step is to calculate an overall stability index using a weighted combination of the individual values. As discussed above, the overall mine stability can be related to many geologic, structural and/or stress factors. In the general case, certain factors may have more influence on the final stability than others; therefore, the final calculation of the overall stability index allows the user to apply various weightings of individual factor indices in order to calculate the final overall stability index.

This module implemented the commonly used weighted average method to calculate the final stability index. For each stability index calculation, a project is defined including all of the critical influence factors imported in this index and their assigned weightings. The final index is computed by using Equation 4.16:

$$
\operatorname{Ind}_{i j}=\frac{\sum_{k=1}^{n}\left(V_{i j}^{k} \times W_{k}\right)}{\sum_{k=1}^{n} W_{k}}
$$

Where: $\operatorname{Ind}_{i j}$ is the stability index at element $(\mathrm{i}, \mathrm{j})$

$k$ is the $\mathrm{k}_{\mathrm{th}}$ influence factor

$n$ is the total number of influence factors in this final index calculation

$V_{i j}^{k}$ is the individual stability index at element $(\mathrm{i}, \mathrm{j})$ for influence factor $\mathrm{k}$

$W_{k}$ is the assigned weight for the influence factor $\mathrm{k}$

Figure 4.11 displays the flowchart of the final stability index calculation. In the process, the final index grid is first initialized. Then for each grid element, the all of individual index are retrieved from individual index grids to perform the calculation. 


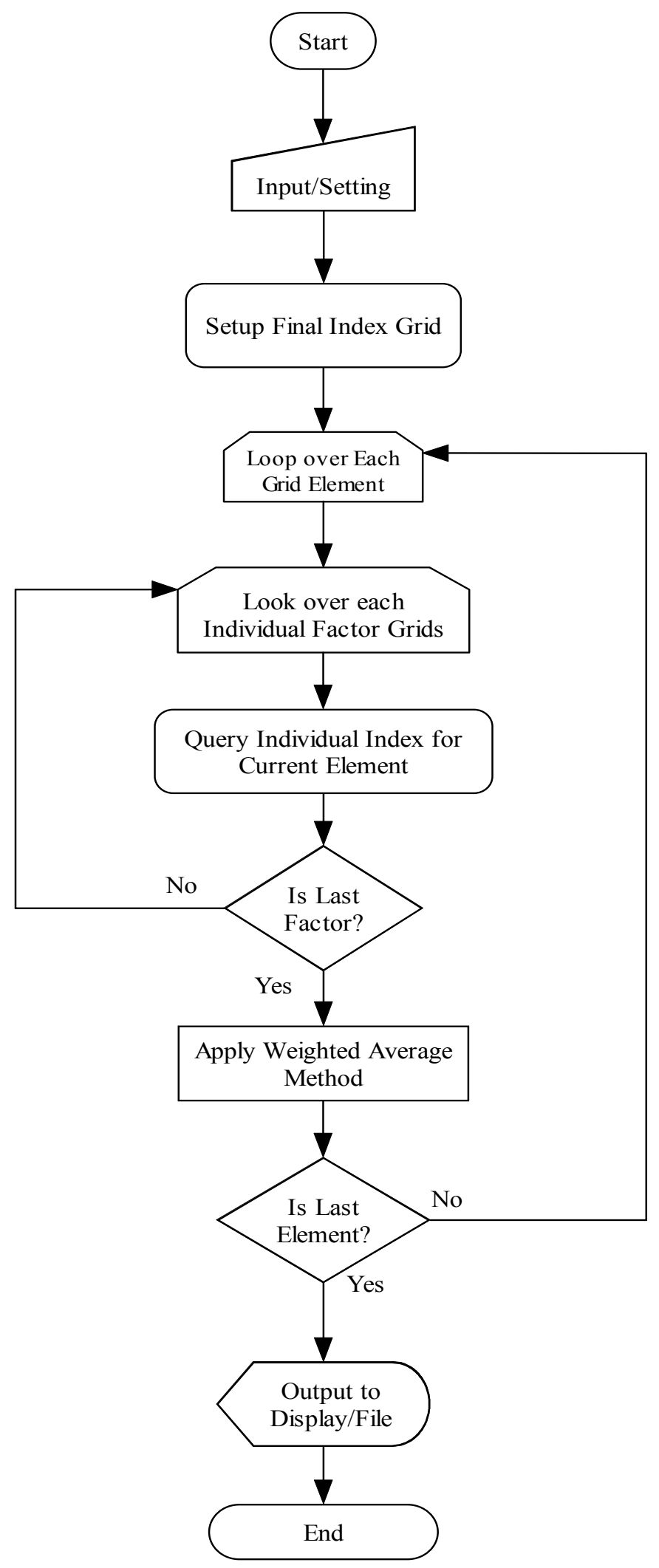

Figure 4.11 Details of final stability index calculation 


\subsubsection{Final Index Mapping Module}

After the final stability index is calculated, it is usual desired to plot the result over the map for analysis. A comprehensive grid utility module has been developed to display the whole grid and its legend into the mine map with a number of different drawing options: such as points, polygons, faces, 3D splines, solid blocks, and hatched areas. A more advanced plotting module is also provided in order to map any grid over the mine entry layout. It means that only the portions of the grid elements directly within the entries are plotted. This drawing is similar to the conventional hazard mapping in which mine engineers record their analysis and observation along the mine entries with different symbols or colors to indicate the hazard/stability distribution.

Main input parameters for this advanced plot module are the final index grid file and pillar polygons in the grid area. Detailed flowchart of this module can be seen in Figure 4.12. The core algorithm for this module determines the remained regions of the grid elements after subtract the regions defined by the pillars. There are three cases in this process:

- If a grid element is entirely outside all pillar dimensions, a standard AutoCAD region object will be defined with the same dimension as the grid cell.

- If a grid element is intersected with any pillar, a region object will be constructed by subtracting the intersection from the grid cell.

- If a grid element is entirely inside any pillar dimension, then no region will be constructed.

It is easy to see that these regions derived from grid elements are the areas of mine openings or entries. The module will plot these regions on the mine map with appropriate colors representing different stability index values. 


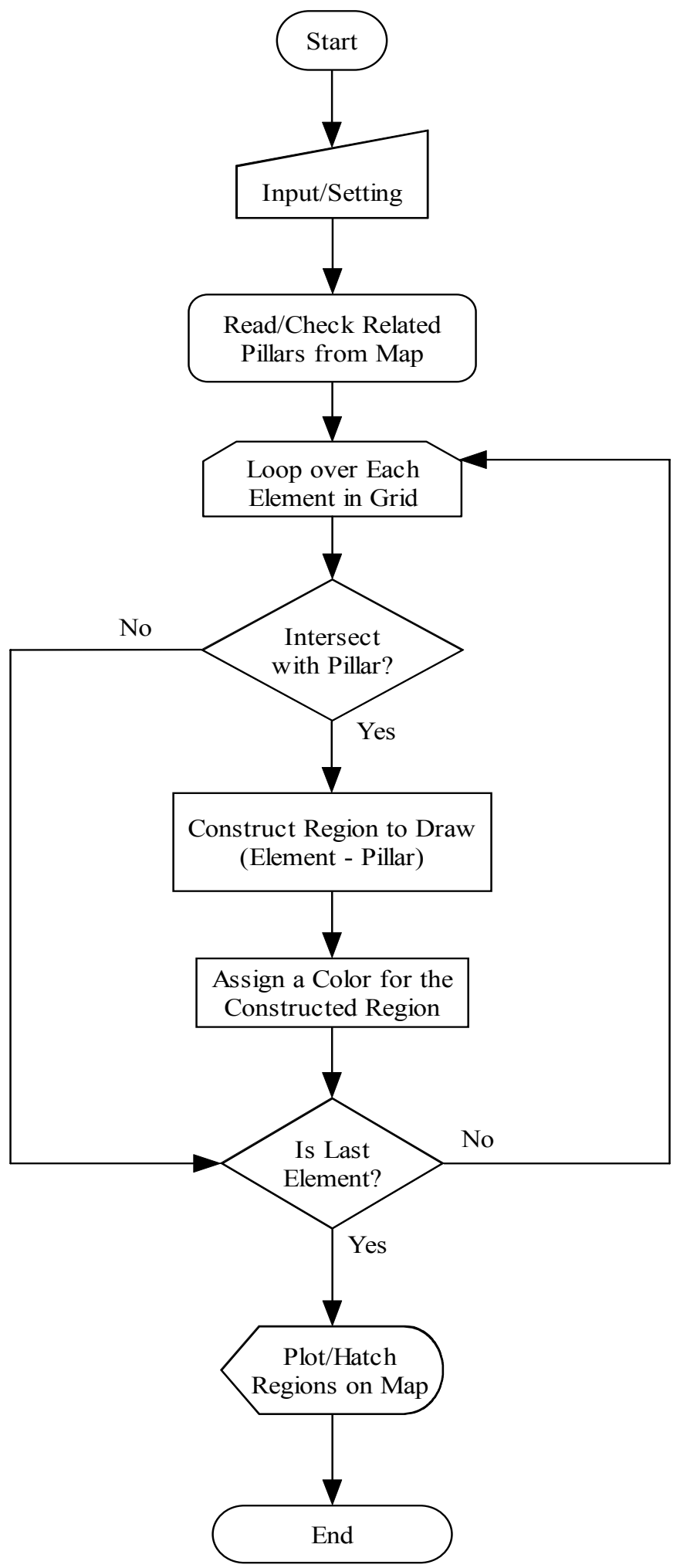

Figure 4.12 Details of stability index mapping module 


\subsection{Summary}

As we stated before, a number of independent modules constituted the stability mapping system. In this chapter, three groups of principal modules have been discussed in detail including their data structure, input data, algorithms and module flowcharts. The geology oriented modules facilitated automatic grid generations for a variety of geology properties and geologic structural features which are imported directly from mine map. On the mine map, these geologic features may have various graphic representations such as point, polyline, contour and closed polygon.

In the stress module, a horizontal stress module considered the influence of the orientation of regional horizontal stress on mine entry stability. At meantime, a number of significant enhancements to LaModel stress analysis have been completed, which include the overburden stress calculation, the automatic seam and topography grid generation, the LaModel stress calculation and output format conversion.

The stability index mapping modules incorporated the capabilities to convert the influence factors into individual stability index by applying various transformation methods, to compute the final stability index by weighting the critical influence factors, and to map the stability index on the mine map.

These modules have generally covered all aspects of a stability analysis and formed the core components of the integrated stability mapping system. 


\section{Chapter 5. Interfaces and Constraints}

\subsection{System Interfaces}

The ultimate outcome of this research is a complete computer program residing inside the AutoCAD/SurvCADD environment. The interface is an extra pull-down menu in AutoCAD/SurvCADD menu bar system. The pull-down menu list contains the accesses to all of the modules/commands developed in the stability mapping system. The menu structure of the stability mapping system is shown in appendix B.1. The selection of a pull-down menu item will invoke the input form to an appropriate/specified module. As a modern program, an advanced graphic user interface is an essential for its success. Fortunately, AutoCAD ObjectARX (see Appendix C.2) extensions for MS Visual C++ have provided a powerful development environment with a rich programming interface to the AutoCAD database (Appendix C.1). This interface allows designing the AutoCAD interactive and/or Windows-style graphic user interfaces with MFC (Microsoft Foundation Class) compatible extensions. By using the ObjectARX MFC extensions, all of the implemented functional modules have the same consistent appearance as standard MS Window forms. The input forms and main input data for the principal stability modules are listed in Appendix B.2. Figure 5.1 demonstrates a typical user interface implemented for the seam grid generation of LaModel inside AutoCAD environment. This interface consists of two tabbed forms. The first form (Figure 5.1(a)) defines the location and dimension of the seam grid, whereas the second (Figure 5.1(b)) is used to input the layer name of seam pillars and specification of the gridding algorithms. The 
graphical inputs can be typed directly from the keyboard or imported interactively from AutoCAD the graphic view into the appropriate boxes (more see Appendix B.2).

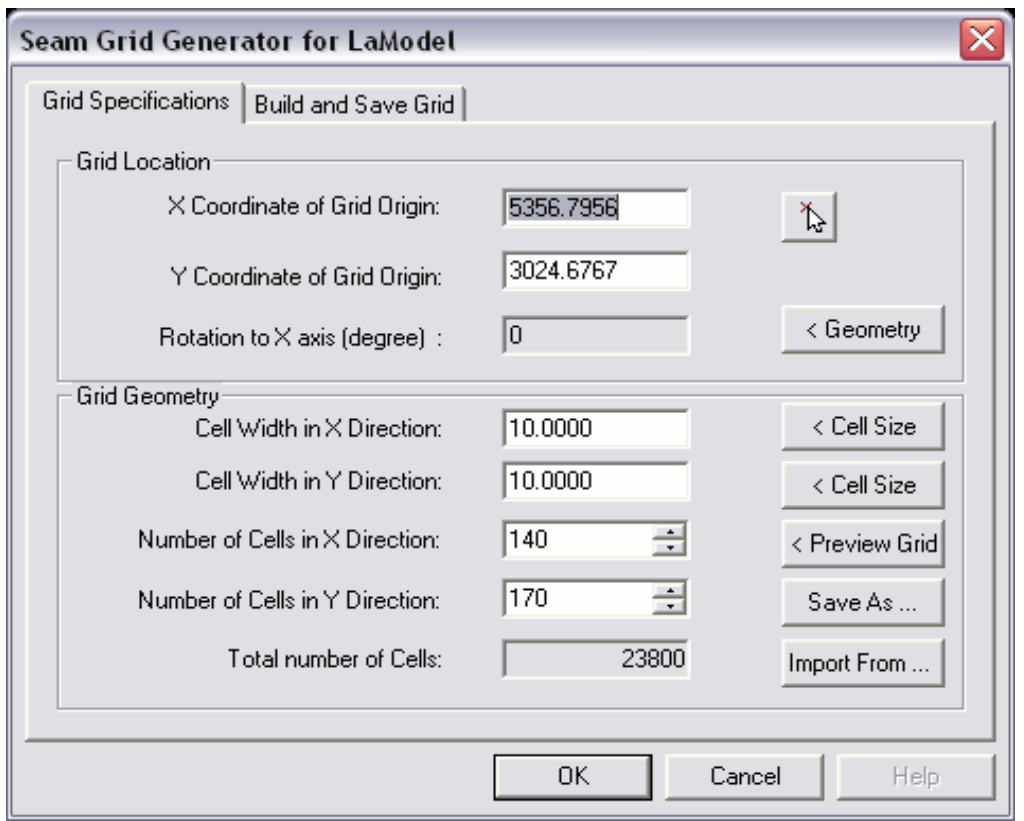

(a)

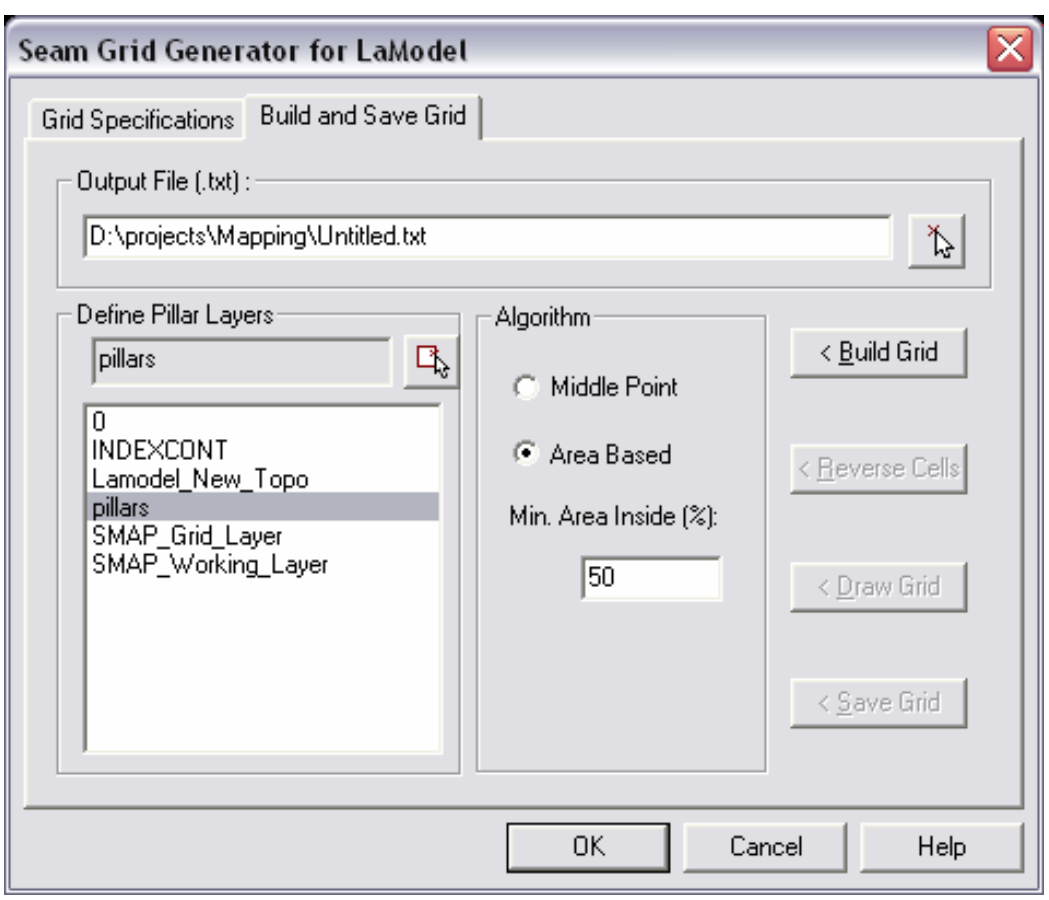

(b)

Figure 5.1 User interface example 


\subsection{Constrains}

The accuracy and practicality of the stability mapping system heavily depends on the quality of input data. For a promising stability mapping analysis, the critical influence factors have to be identified and sufficiently detailed information of these critical influence factors needs to be accessible. To identify the critical influence factors, previous experiences and field observations at mines with the same or similar geologic conditions can provide an initial estimation. The stability analysis can be refined with increasing detail on the influence factors from further field exploitation and mining operation.

Also, the purity of the input data represented in the mine map also affects whether a stability module can function properly. As discussed in previous chapters, in many cases, the input data are extracted automatically from specified map layers. This implies that only the graphical representations of that particular influence factor should be stored in that particular layer, and the representations must be a compatible drawing type. If data with improper graphic types are passed into the stability module, this information may be ignored or cause unpredictable results. For example, in all mapping modules, all "Text" objects in the layers will be ignored; but all "Polyline" objects will be considered differently in different modules. In the liner structural feature module, a "Polyline" is often a series of connected lines representing the center line of the features. If such an open-ends "Polyline" is passed to the area-based geology module which requires a closed "Polyline" as the influence boundary, the module will issue a warning. Ignoring the warning may cause unpredictable results. 
On the other hand, since the stability system is designed to execute entirely inside the AutoCAD environment, it will inherit the same constraints as AutoCAD. For instance, a map file from a new version may not be readable by an older version of AutoCAD and a new version of AutoCAD may not able to load in the application compiled with an older version of AutoCAD. So some degree of portability between the possible AutoCAD environments has been considered during the implementation of the stability mapping system. The strategies to maintain optimum portability have included separating the user interfaces from the computational algorithm components, employing standard library functions, applying commonly-used window components for user interface/input form, and documenting the version dependences as clear as possible.

The computer system requirements for the stability mapping modules depend on the version of AutoCAD. Currently, the stability mapping system is designed to be compatible with the present AutoCAD Versions including version 2002, 2004, and 2005. With the release of a new version of AutoCAD, the stability system would be either fully compatible or could be easily migrate to the new system with minimal efforts.

However, it should be noted that AutoCAD LT is a special release of the AutoCAD software without capabilities in 3D functionality, customization (LISP, ARX, VBA), presentation graphics, etc. Thus the stability mapping system will not load in any LT version of AutoCAD. 


\section{Chapter 6. Case Studies and System Validation}

\subsection{Introduction}

The proposed integrated stability mapping system has been developed into a simple, user-friendly window-based program. In order to evaluate the functionalities of the modules and to demonstrate the application of the system, two simple case studies have been performed with actual field data. Through the practical application of the system to these case studies, a number of refinements and enhancements have been made to the system design and implementation. This chapter will discuss these two case studies in detail. The first case study was conducted in a mine located in Greene County, Pennsylvania. In this mine, in addition to the overburden stress, a fracture zone caused by stream valley was also considered as one of the critical influence factors, since its observable high impact on mine stability underground. The second case study was conducted at a deep longwall mine where researchers had performed field observation of the entry stability in the past. The resultant pillar/rib conditions had been classified on the mine map, and provided an ideal opportunity to compare the calculated stability index with reality for the case study.

\subsection{The Case study one}

The first case study mine is operating in the Pittsburgh seam using longwall mining method. At this site, the coal seam has a thickness of 6 to $7.5 \mathrm{ft}$ and a nearly flat dip between 0 and $3 \%$ to the southeast. The cover of the coal seam is between 600 and 
$800 \mathrm{ft}$. As in the first case study, for simplicity of the demonstration, only a small section of a longwall panel was analyzed in this study (see Figure 6.1).

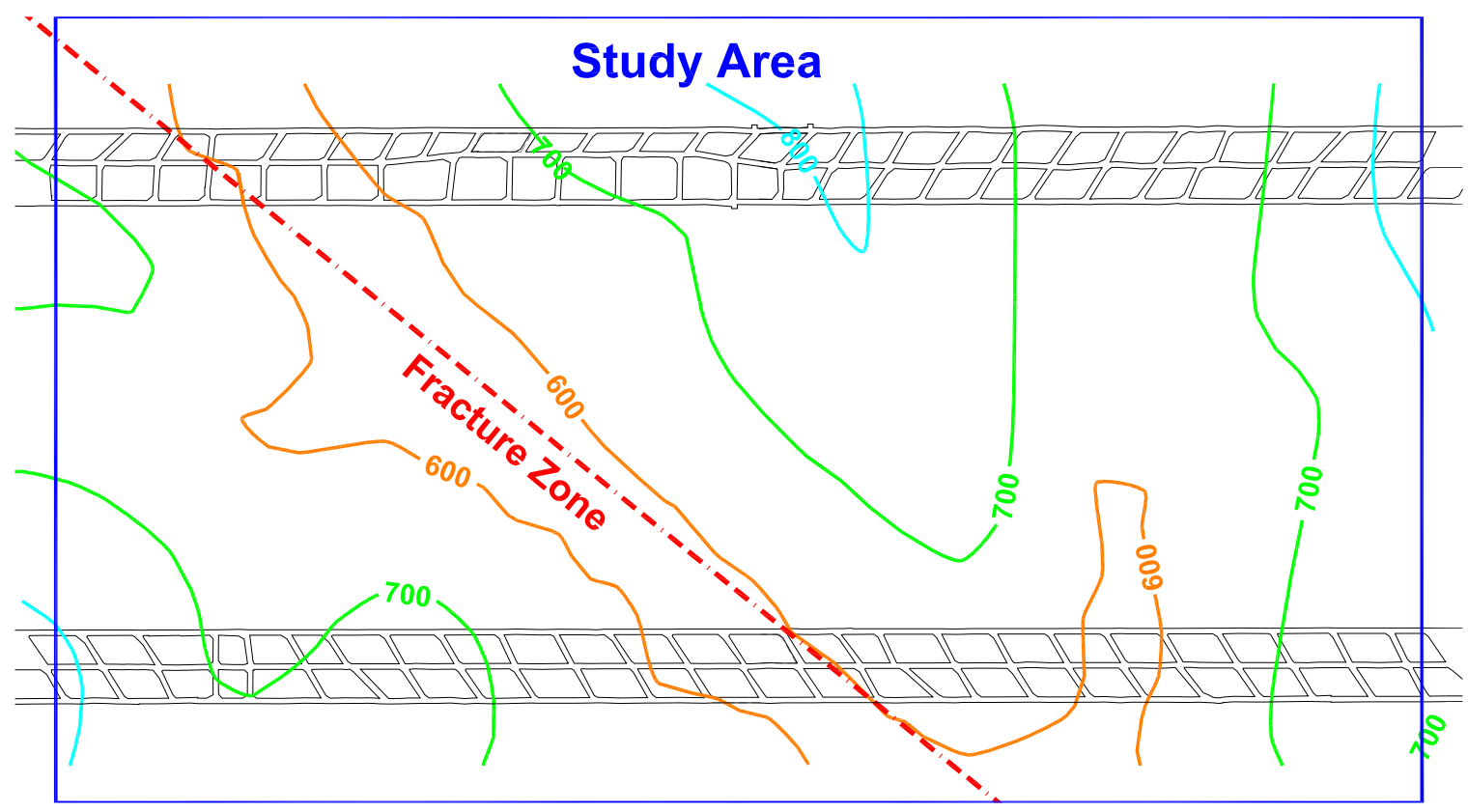

Figure 6.1 Map of the first case study area

In this case study, two parameters were considered as critical factors influencing the roof stability, namely a fracture zone under a major stream valley and the overburden stresses. The study area was modeled using a 400 by 230 grid (totally 92,000 elements) with 10 by $10 \mathrm{ft}$ element. The fracture zone was presented with its center line in the mine map, and its influence on mine stability decreases with the increasing of distance to the center line. For this type of structural feature, the linear structure module of the system is appropriate to calculate the individual stability index. It was assumed in this case that the influence distance of the fracture zone was $300 \mathrm{ft}$. This implies that the influence of the fracture is linearly distributed with the $300 \mathrm{ft}$ distance to the center of the fracture at both sides, while there is no impact for areas outside the $300 \mathrm{ft}$ influence range. Figure 6.2 
shows the individual stability index calculated for this fracture zone with the highest index (100) along the center line of the fracture.

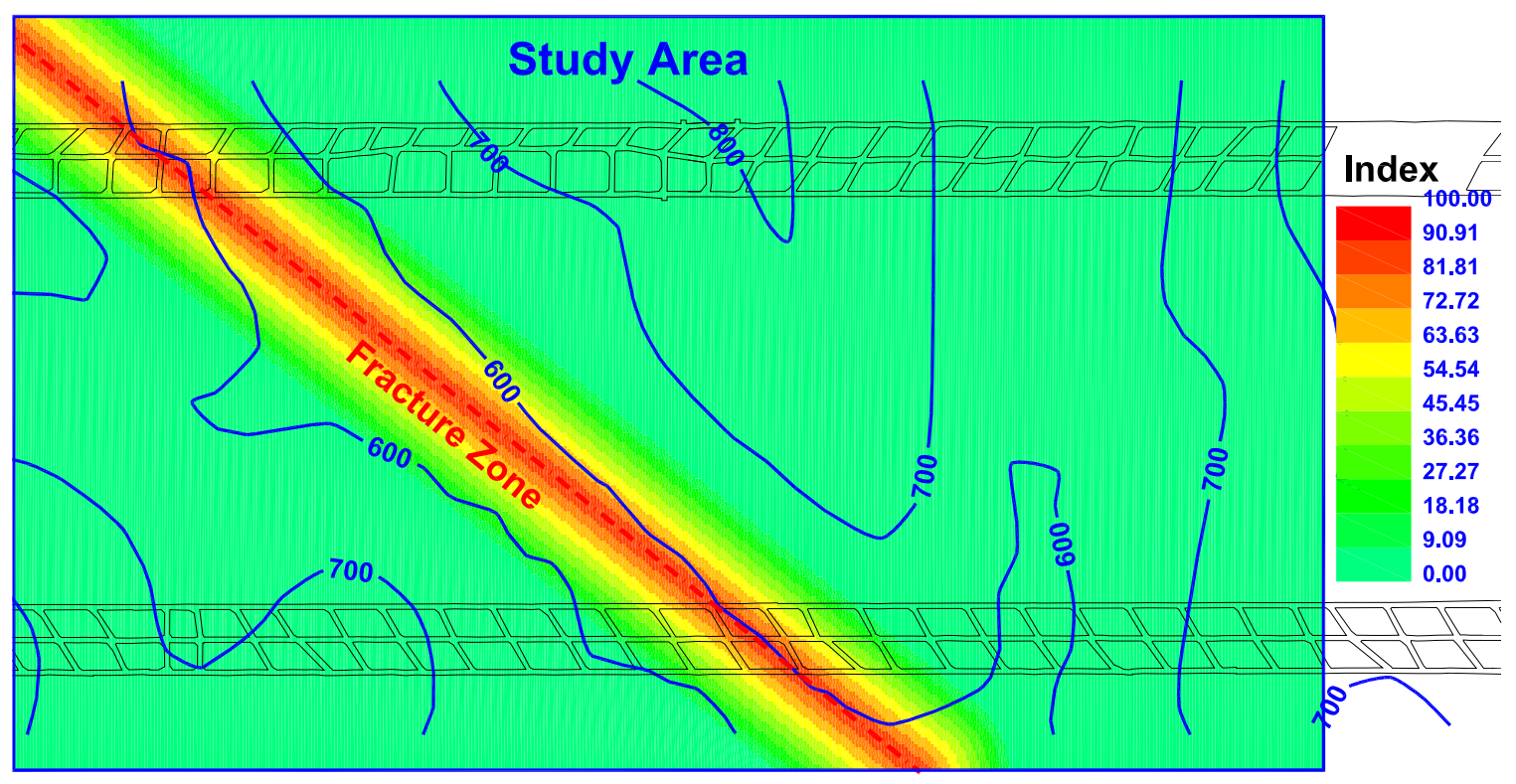

Figure 6.2 Stability index for the fracture zone

On the other hand, the overburden stresses were calculated using the overburden stress module derived from original LaModel stress calculation. A pre-defined overburden thickness grid was automatically generated by applying the contour based geology module with a grid dimension of 105 by 55 (totally 5775 ) elements and 50 by 50 $\mathrm{ft}$ element size. The overburden stresses calculated from this overburden thickness grid over the case study area are shown in Figure 6.3. 


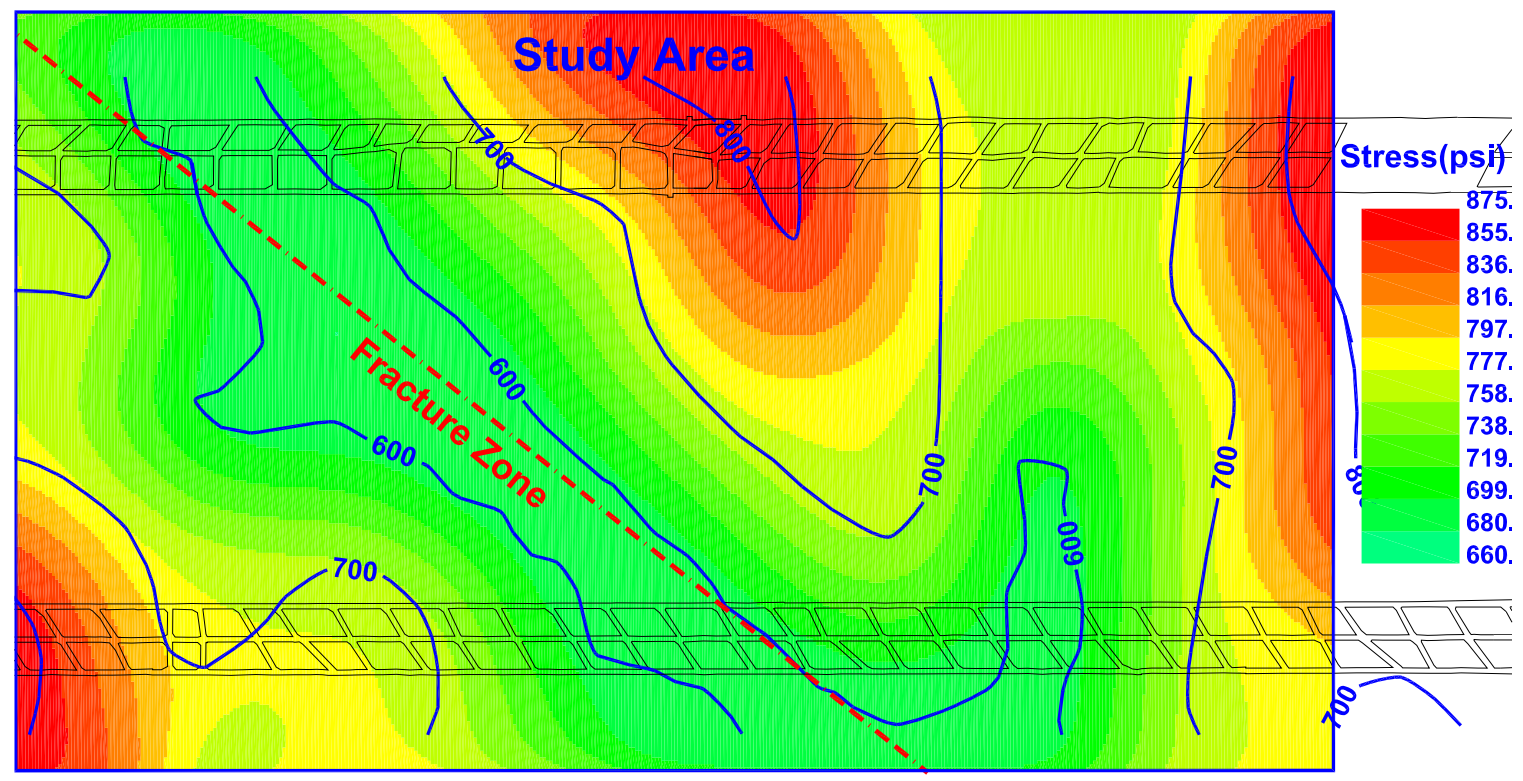

Figure 6.3 Plot of overburden stresses

The overburden stresses with a stress range from 600 psi to 875 psi then were converted into an individual stress stability index with the index range from 0 to 100 by using a linear transformation. Since in this case the influence of the fracture zone was already normalized to 0 to 100 during creation, the two individual factors were ready to be combined into the final stability index. A 50\% weighting factor was applied to each influence factor, so the overburden stress and the fracture zone influence were equally combined into the stability index. Figure 6.4 shows a plot of the composite stability index distribution. It can be seen that the both overburden stress and the fracture zone influence are finely combined. In particular, the width of the fracture zone influence is increasing with the increasing in the overburden. 


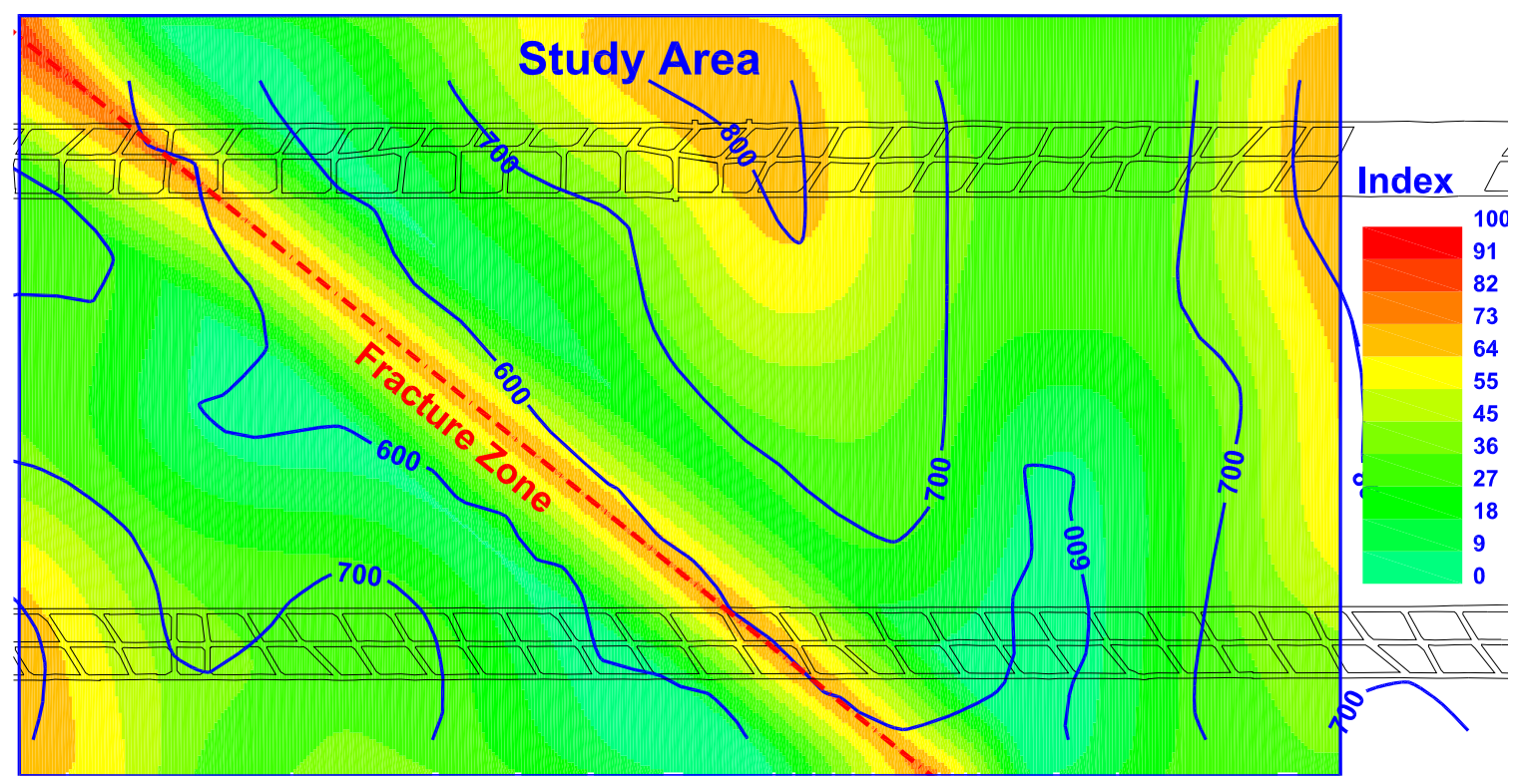

Figure 6.4 Plot of final stability index

At this mine, the geologist goes underground and map roof features such as cutters, cracks, spalling, etc. onto an AutoCAD mine map (see Figure 2.1). Using this map and the linear geologic feature module of the system, an index of the density of these roof features was created and then mapped onto the gateroad entries. A side by side comparison between the observed roof condition and the calculated stability index is shown in figure 6.5 .
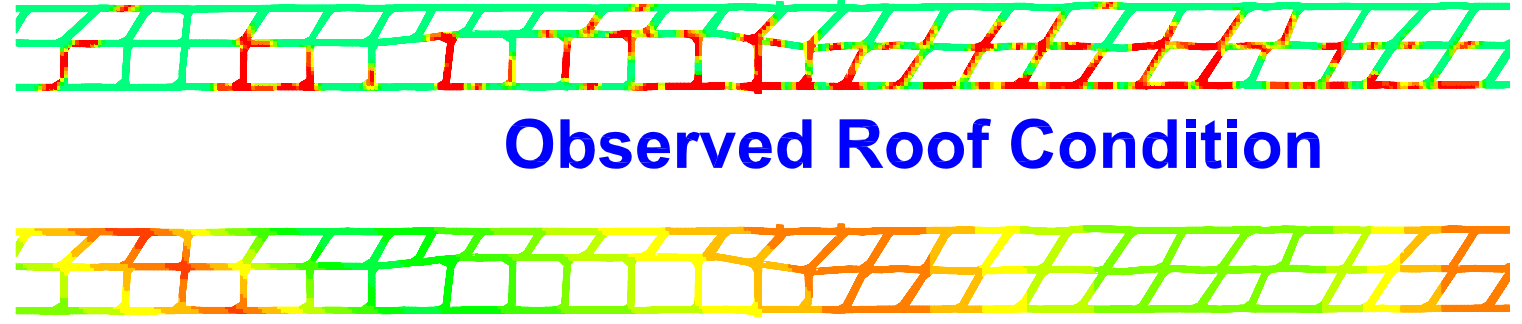

\section{Stability Index Mapping}

Figure 6.5 Comparison of observed roof condition with stability index 
Although only two influence factors were included in this simple case study, some weak correlation between the calculated stability index and the observed roof condition can be seen in the comparison. It is anticipated that through incorporating the site specific geology influence factors into the analysis, more accurate stability index calculation would be obtained.

\subsection{The Case study two}

\subsubsection{Background Information}

This case study is a single seam, longwall, coal mine scenario with high topographic relief in Eastern Utah, situated in the area of the Book Cliffs and the eastern Wasatch Plateau in the northwest corner of the Colorado Plateau (Heasley, 2001). The geology at this location is fairly typical of the Uinta coal basin with various sedimentary layers of sandstones, a combination of shale and siltstone, and coal beds. The coal seams are located in the Blackhawk Formation of the Mesa Verde Group (see Figure 6.6). The study mine is primarily in the Castlegate " $\mathrm{D}$ " seam. The coalbed at the mine ranges from 8 to $20 \mathrm{ft}$ in thickness with an extraction thickness of 8 to $10 \mathrm{ft}$. the geology immediately above and below the seam consists of thinner $(<10 \mathrm{ft})$ layers of delta deposited siltstones, mudstones, shales, sandstones and coal. Above the coal bearing portion of the Blackhawk Formation, approximately $500-600 \mathrm{ft}$ of braided stream deposits with numerous lenticular sandstone channels occur. These braided and lenticular deposits make up the immediate and main roof of the mine. 


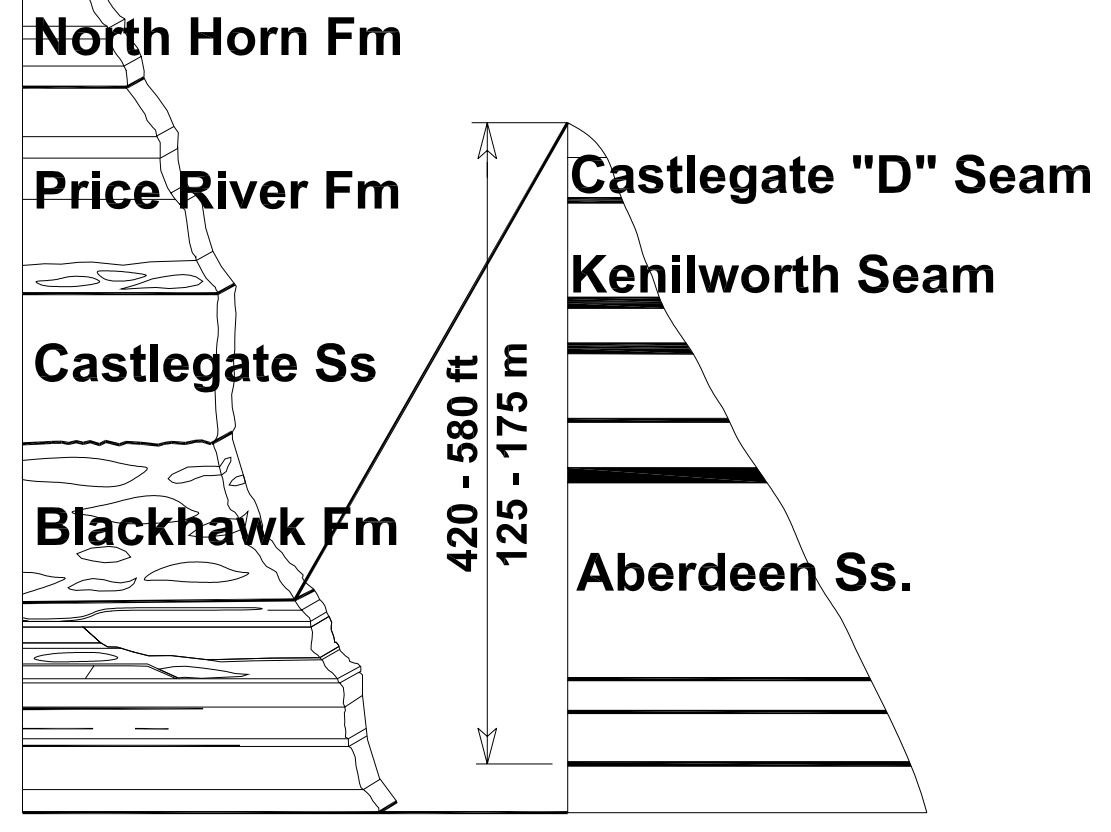

Figure 6.6 Generalized stratigraphy

Unconformably overlying the Blackhawk Formation is the Castlegate Sandstone. The Castlegate is a massive, cliff forming sandstone that is 400 to $600 \mathrm{ft}$ thick with the lower $300 \mathrm{ft}$ being more compact and massive than the upper portion of the unit. In the mine area, the Castlegate Sandstone lies approximately $680 \mathrm{ft}$ above the " $\mathrm{D}$ " seam. Overlying the Castlegate is the Price River formation, consisting primarily of sandstone with interbedded conglomerates and sandstones. The Price River formation is also about $600 \mathrm{ft}$ in thickness. The uppermost rocks exposed at the site are lacustrine deposits of North Horn formation, consisting of interbedded claystones, mudstones, siltstones, and sandstones. Overall, the overburden thickness at this mine varies dramatically from $500 \mathrm{ft}$ up to $3000 \mathrm{ft}$ (see Figure 6.7) (Heasley, 2001).

To limit the scope of the work and make use of available information, the case study was conducted on a small section of longwall panel 1 with an area of 3500 by 1400 
feet (see Figure 6.7 and 6.8). At the study area, the overburden thickness ranged from 1300 to 2600 feet with an average around 1950 feet. The case study area was modeled using a 350 by 140 (total 49,000 elements) grid with a 10 by $10 \mathrm{ft}$ element.

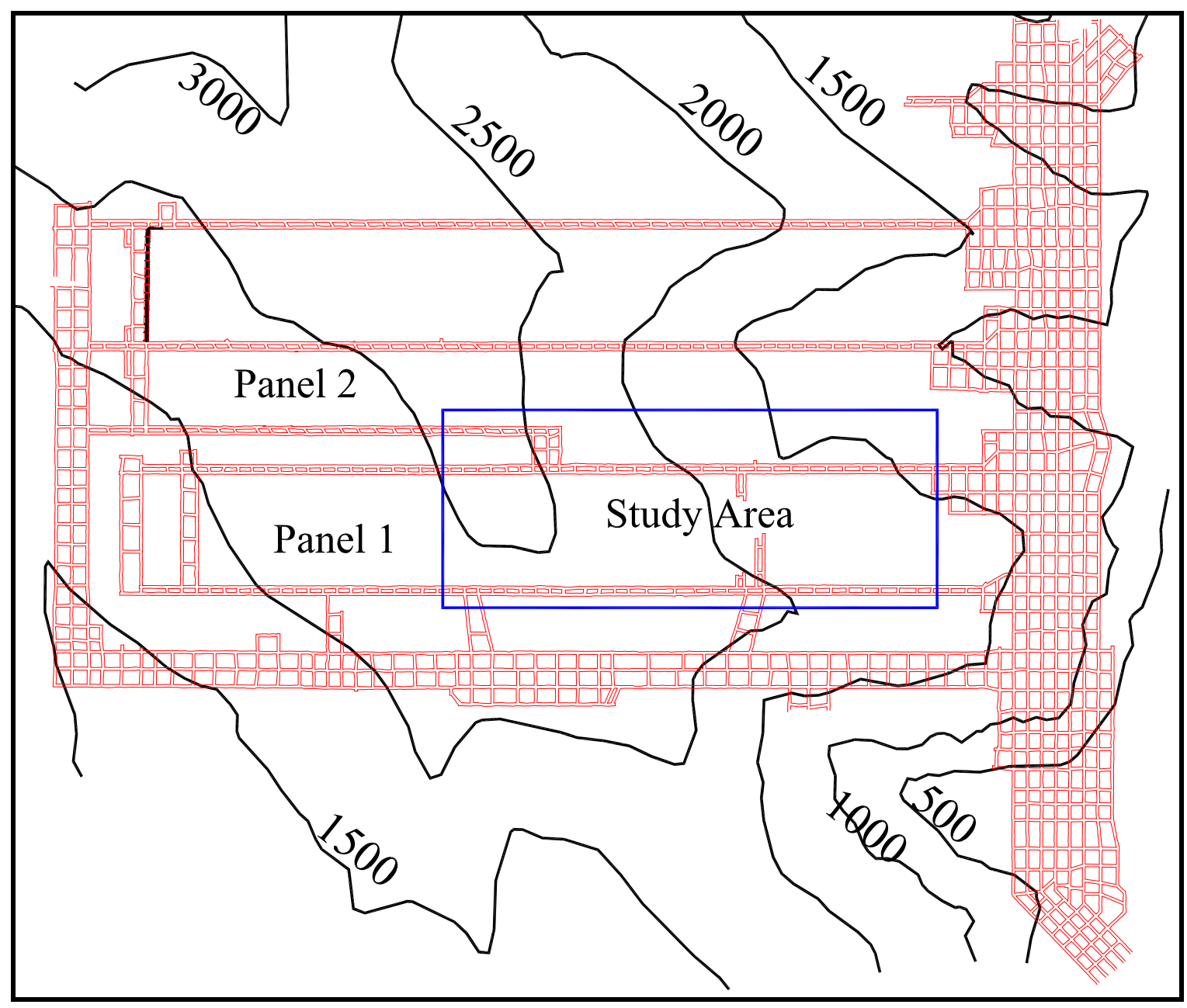

Figure 6.7 Plan view with mine layout, overburden, and the location of the case study. 


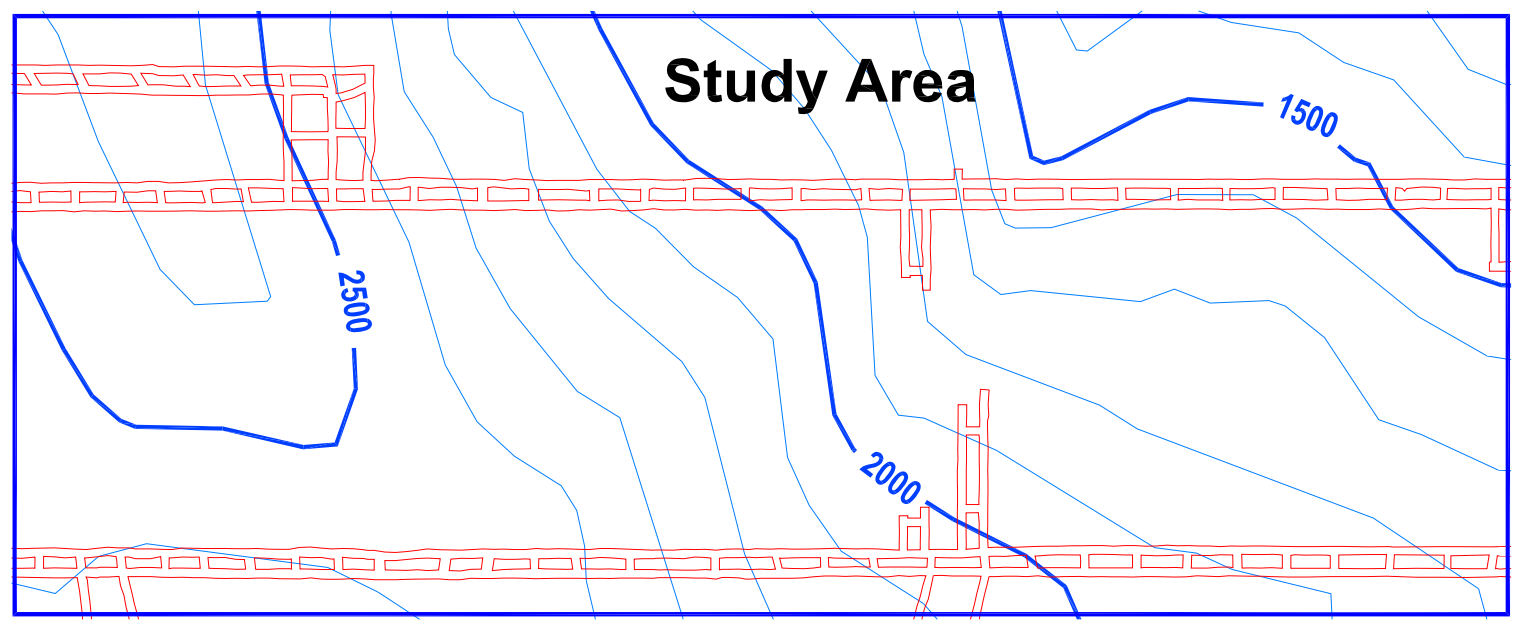

Figure 6.8 Map of study area.

\subsubsection{Stability Mapping Process}

The first step of a stability mapping analysis is to identify the critical influence factors which impact the mine stability the most. Obviously, for this deep longwall mine, the magnitude and variability of the overburden thickness would be an important aspect that should be considered in the analysis. Also, according to previous mining and ground control experiences in this mine, it was found that the lenticular sandstone channels in the immediate roof have significant influence on the final pillar/rib stability of the entries. In general, the thick sandstone layers closer to the seam generate higher rib stresses and worse roof condition. To fully explore the influence of the sandstone on mine stability, the thickness of sandstone in the immediate roof and the interval between the sandstone lenses and the seam are both analyzed in this case study.

The geology data were taken from underground borehole logs extracted along the longwall entries, with total of 67 boreholes processed in the study. The thicknesses of the sandstone lenses and the thickness of the immediate roof interval were imported into the 
mine map using SurvCADD module. The geology modules from SurvCADD were applied to grid and contour the borehole data (see Figure 6.9).

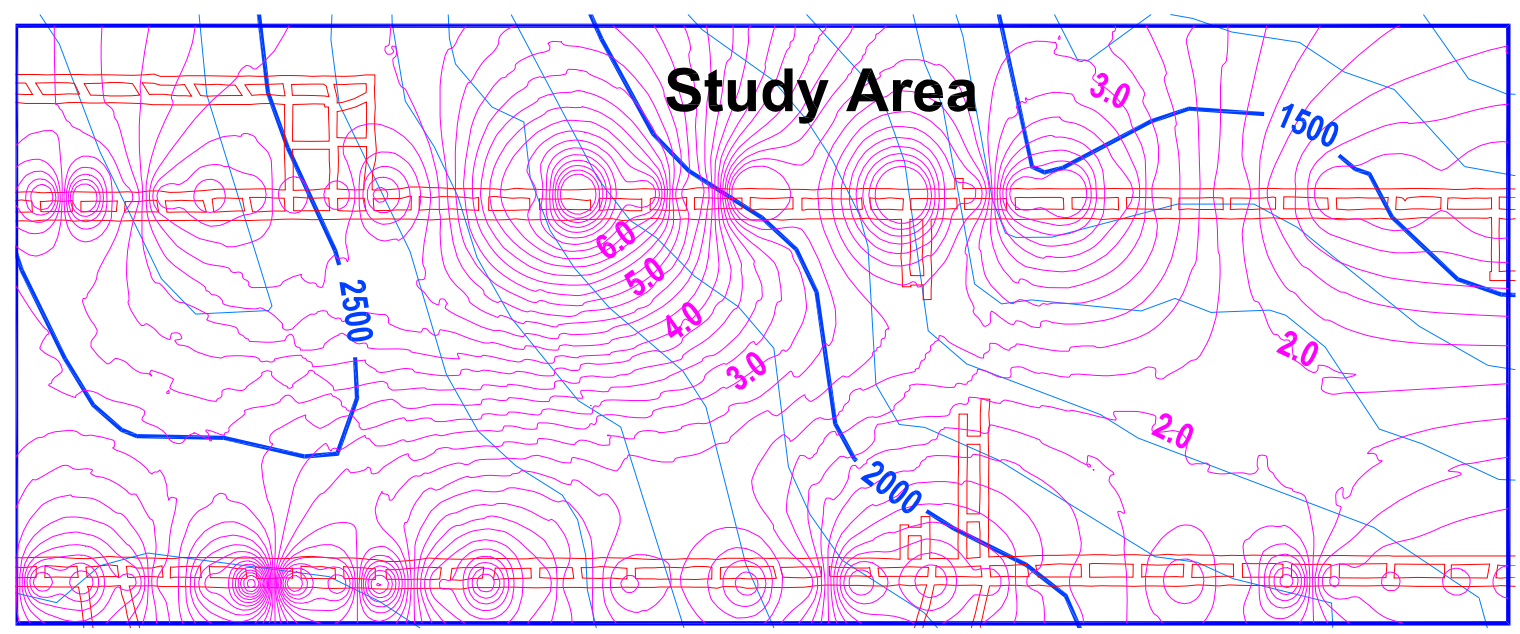

Figure 6.9 Contour of the sandstone thickness within first $10 \mathrm{ft}$

In this mine, experience has indicated that the presence of sandstone lenses within the first $10 \mathrm{ft}$ above the seam often impact the roof conditions. Therefore, to quantify the influence of the sandstone, the percentage of the sandstone in the first $10 \mathrm{ft}$ of the roof was calculated. The resultant grid is plot in Figure 6.10 to show the distribution of the sandstone's existence.

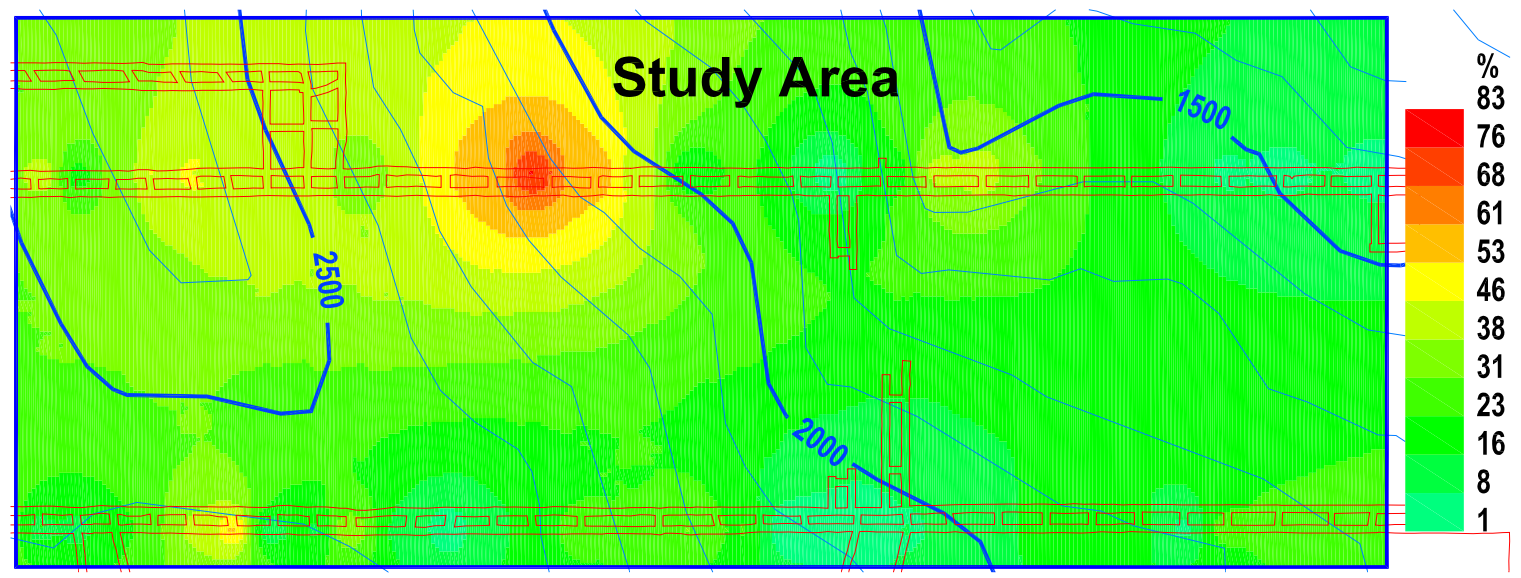

Figure 6.10 Sandstone percentage in the first $10 \mathrm{ft}$ roof. 
Since in this case the higher percentage of sandstone implies a worse roof condition, therefore the sandstone percentage was converted into an individual sandstone thickness index by applying a linear transformation. As a result, the grid elements with highest percentage of 83 will have a stability index of 100 , whereas the lowest elements were assigned an index of 0 .

Similarly, the interval thickness between the seam and the sandstone was gridded by using SurvCADD mining modules (see Figure 6.11 and Figure 6.12).

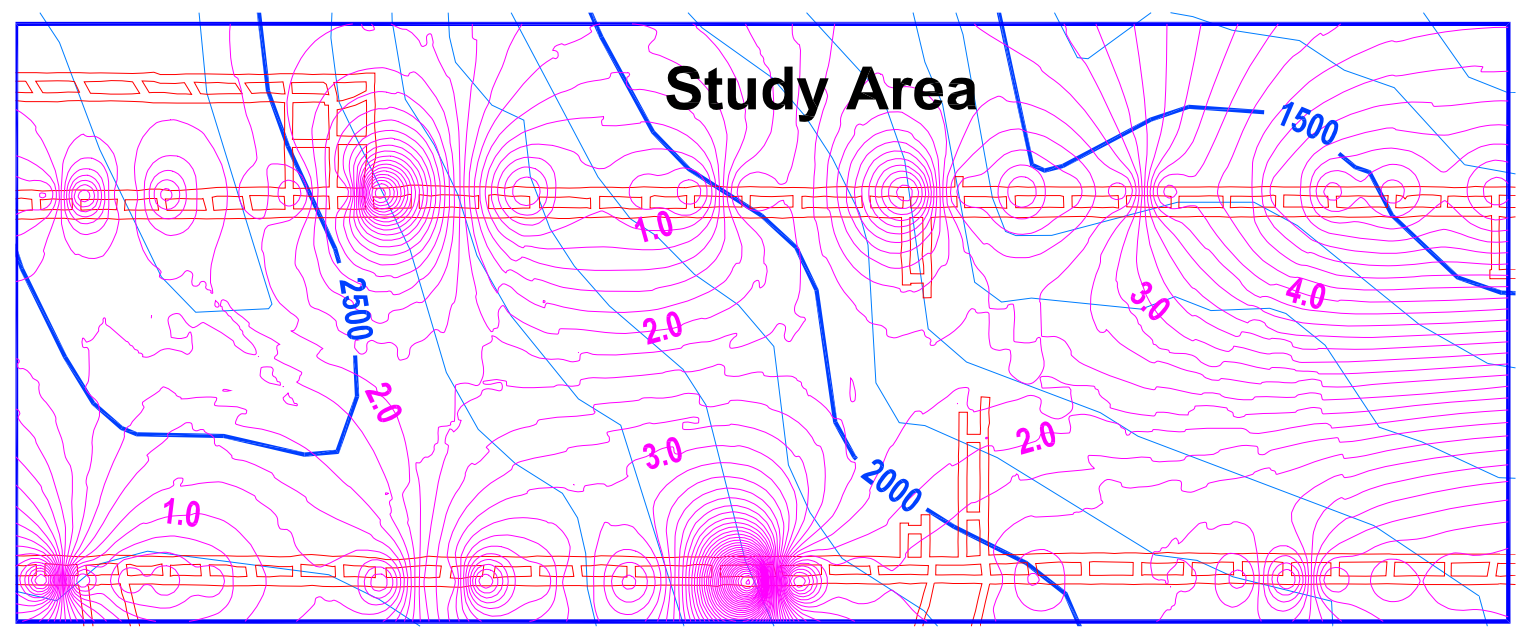

Figure 6.11 Contour of interval thickness

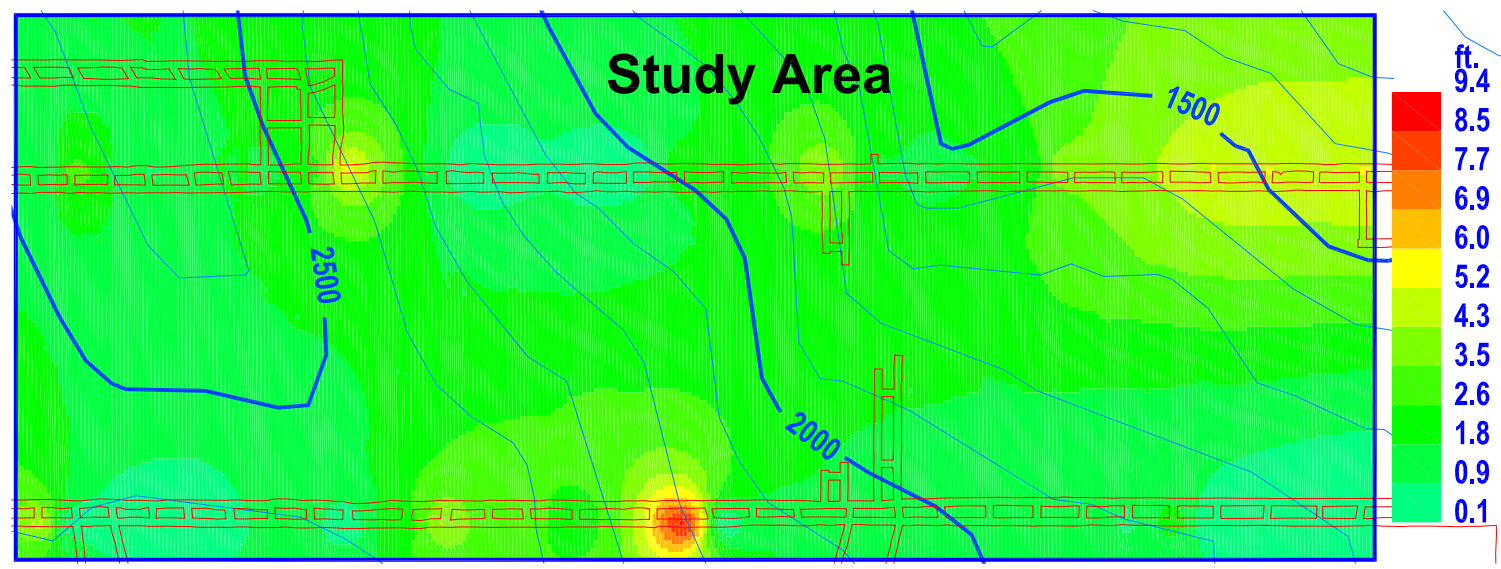

Figure 6.12 Thickness of the interval from seam to sandstone (ft) 
From the gridding results, it was seen that the interval thickness ranges from 0.2 to $9.4 \mathrm{ft}$ (Figure 6.12). It is desired now to translate this interval into an individual index grid that ranges from 0 to 100 . Since a larger interval thickness implies a more stable condition with a smaller stability index value, the inverse linear transformation function would be appropriate for this conversion. By applying the lower and upper bounds set at 0.1 and 9.4 respectively in the inverse linear transformation module, the stability index for the interval thickness was mapped from the 0.1 to $9.4 \mathrm{ft}$ thickness range into a 100 to 0 index range (see Figure 6.13).

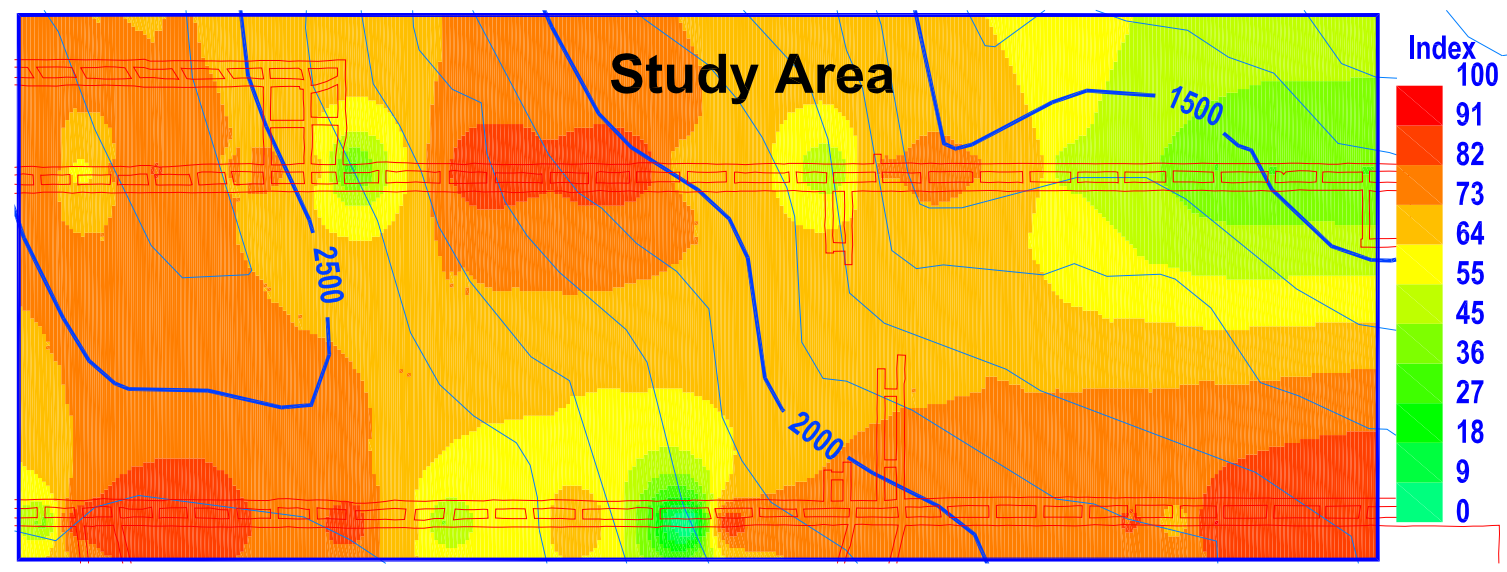

Figure 6.13 Index plot for the interval thickness

To illustrate the step transformation, it could be assumed that at this property the stability problem typically only occur when the interval thickness is less than $2 \mathrm{ft}$. in this case, the inverse step transformation function would be used with the step set at $2 \mathrm{ft}$. therefore, all of the thickness values above $2 \mathrm{ft}$ would be transformed to an index of 0 and all of the values below $2 \mathrm{ft}$ would be transformed to an index of 100 (see Figure 6.14). 


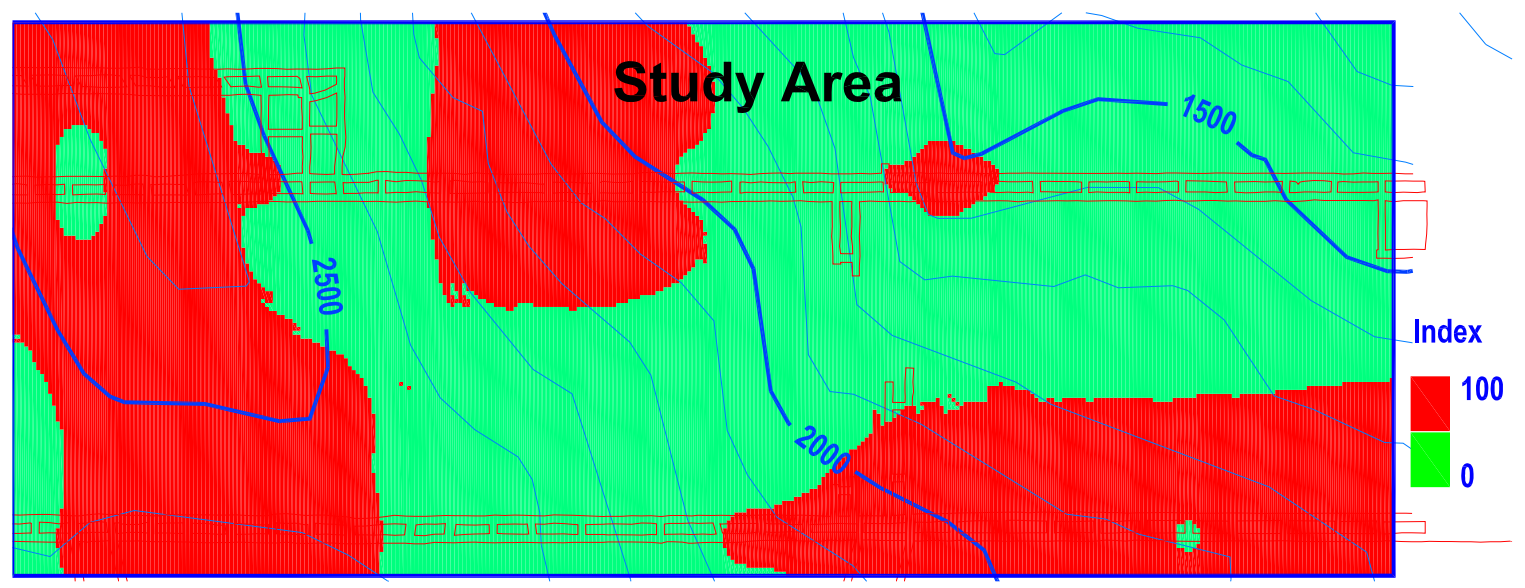

Figure 6.14 Index plot with an inverse step transformation

In order to calculate the overburden stresses, an overburden thickness grid has to first be generated from the overburden thickness contours by applying the geology contour module of the stability system. For accurately projecting the stresses down to the mine seam level, it is generally required that the overburden grid would be larger than the seam grid, but can have less dense elements (Heasley, 1998). An overburden grid dimensioned at 100 by 55 elements with an element size of 50 by $50 \mathrm{ft}$ was used in this case study. After the overburden grid was automatically generated from the contours, it was then imported into the laminated strata calculation module built into the stability mapping system. By using this laminated strata calculation for the vertical seam stress, the overburden forces are softened or evenly distributed so as to more accurately model the true mechanics of the overburden. Figure 6.15 shows the final overburden stress calculated for this case study area. Similar to the thickness of sandstone channels, the values of overburden stresses are generally direct proportional to the stability index, thus the linear transformation approach was used to convert the overburden stress (1425 to 2824 psi) into individual stability index (1 to 100$)$. 


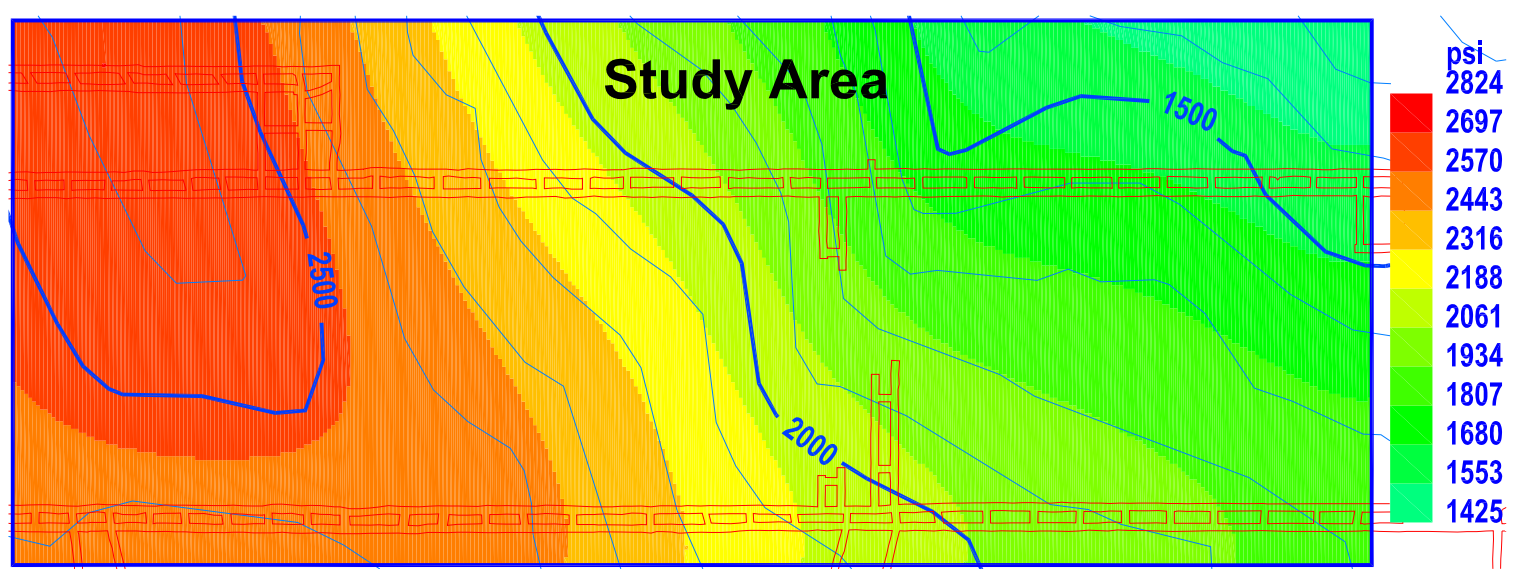

Figure 6.15 Plot of overburden stresses

\subsubsection{Index Mapping and Field Verification}

After the individual stability indices for the critical influence factors have been developed (three influence factors in this case study), they are ready to be combined into the final stability index. The simplest and often used method in most cases is the weighting process. In this approach, each influence factor was assigned a weight according to its perceived contribution to the mine stability. The more an influence factor impacts the final stability, the higher weight should be applied to its individual index. In practice, accurate weighting factors for each influence factor should come from the past field experience of the mine or the investigation of a similar mining setting. However for a preliminary analysis, equally weighting of all influence factors would be a reasonable start point. With increased confidence gained from further exploration and operation, the weighting process could be refined to better reflect the enhanced knowledge of the mining area. In this case study, an equal weighting factor of 33 was used for each influence factor (see Figure 6.16). 


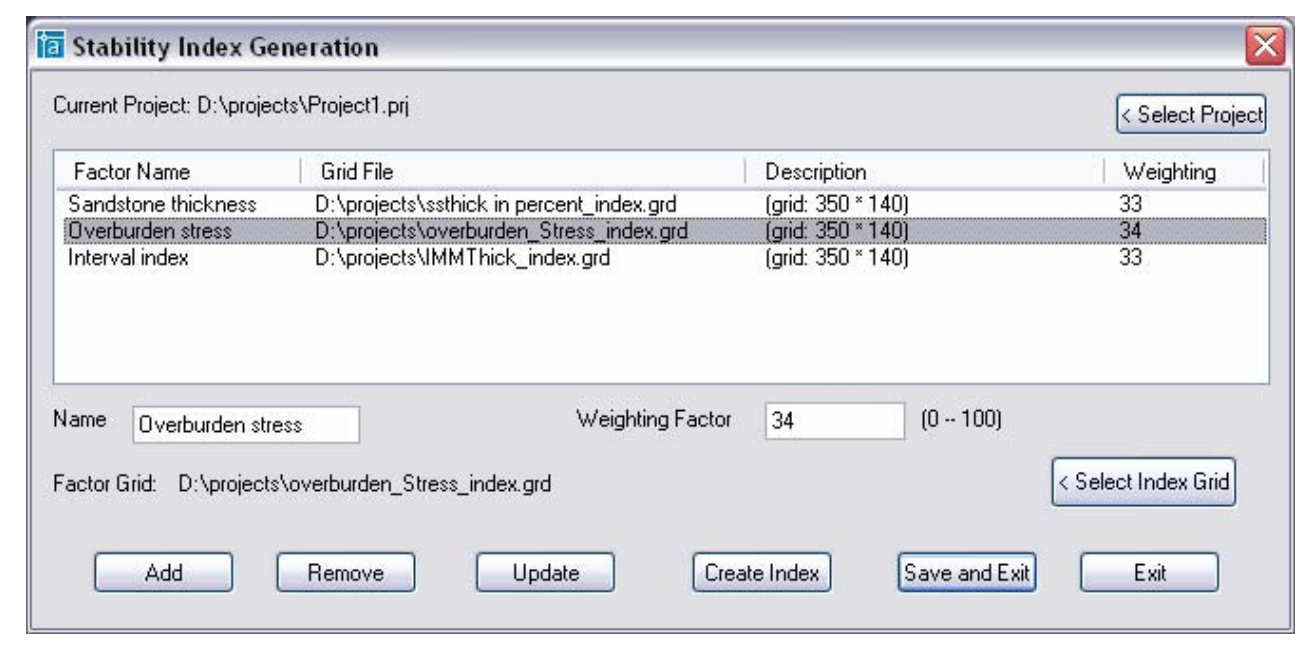

Figure 6.16 Stability index with equal weights

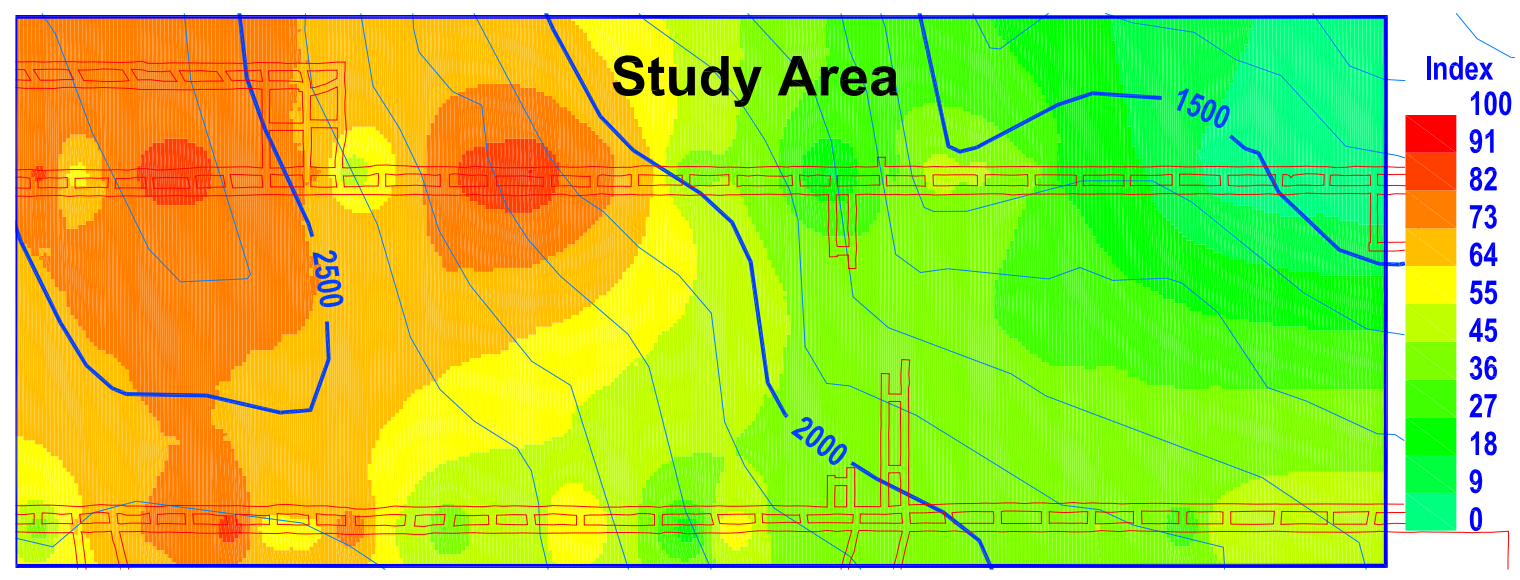

Figure 6.17 Plot of the final stability index

The resultant final stability index can be seen in Figure 6.17. It can be seen how both the overburden stress and geologic influences are combined in the final index. In this stability index output plot, the areas with hot colors (more reddish) are less stable than the areas with cool colors (more greenish).

At this mine, during the entry development and mining operation, the underground roof and rib conditions were stress mapped onto an AutoCAD mine map. An actual observed roof and rib rating map for the upper gateroad was available (see Figure 6.18). In this map, the ribs with a higher rating (up to 4) experienced worse 
conditions than the ribs with lower rating. A comparison between this observed pillar/rib condition and the stability index calculated from the sandstone thickness, sandstone interval, and overburden stress is shown in Figure 6.18.

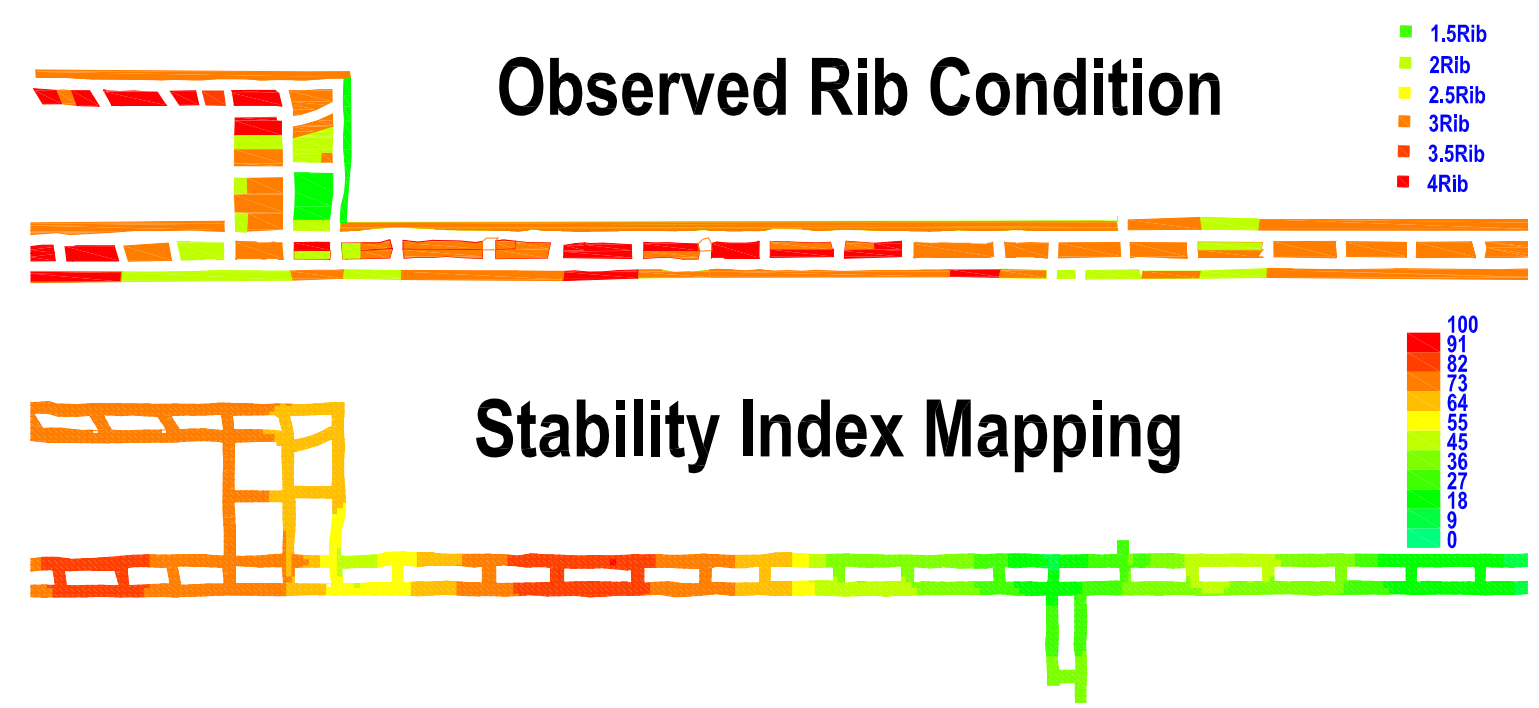

Figure 6.18 Comparison of observed pillar/rib condition with stability index.

In this Figure, it is clearly shown that there appears to be a strong correlation between the calculated stability index and the observed conditions, especially within the high index areas where roof and/or rib problems had been experienced. Although the observed correlation has been pretty good, it must be remembered that this case study was only a very initial stability analysis for the purpose of demonstration. In practice, with increased experience in a particular mine condition, more detailed geology information and/or other critical site influence factors could be included, and the influence factor transformation and weighting process could be optimized into a more through and accurate stability analysis. 


\subsection{Application guidance}

Although the integrated stability mapping system has provided comprehensive functionalities in helping perform complex mine stability analysis, there are two important procedures that are necessary to be emphasized. These two procedures may have significant impacts on the accuracy of the final stability estimation. One of the procedures is to identify the critical influence factors for specific mine sites, another is to assign the weight for each individual influence factor. As we discussed before, due to the variety of the geologic conditions and mining methods, The solution for these problems would be rather site specific and generally requires extensive on-site investigations and experiences in an mine with the same or/and similar mining conditions. In order to provide a general reference to initial system users, the influence factors and their weights used in the case studies and the literatures reviewed of the dissertation was summarized in Table 6-1.

Table 6-1 Case summary for stability influence factors

\begin{tabular}{|c|l|l|l|}
\hline References & \multicolumn{1}{|c|}{ Factors } & \multicolumn{1}{|c|}{ Location } & Weighting \\
\hline Case 1 & $\begin{array}{l}\text { Stream valley } \\
\text { Overburden stress }\end{array}$ & Pittsburgh seam & Equal weights \\
\hline Case 2 & $\begin{array}{l}\text { Sandstone lenses } \\
\text { Interval between sandstone } \\
\text { and seam } \\
\text { Overburden stress }\end{array}$ & Eastern Utah & Equal weights \\
Stankus & $\begin{array}{l}\text { Sandston channels } \\
\text { Mica in the sandstone } \\
\text { Tectonic stress } \\
\text { Stream valley }\end{array}$ & Enlow Fork & Equal weights \\
\hline
\end{tabular}




\begin{tabular}{|c|c|c|c|}
\hline $\begin{array}{c}\text { Newman } \\
2001\end{array}$ & $\begin{array}{l}\text { Roof fractures } \\
\text { Calcite veins } \\
\text { Pillar strength } \\
\text { Stress interaction }\end{array}$ & Lexington Limestone & \\
\hline $\begin{array}{r}\text { Keim } \\
1999\end{array}$ & $\begin{array}{l}\text { Overburden thickness } \\
\text { Orientation of roof falls } \\
\text { Horizontal stress } \\
\text { Sandstone channels } \\
\text { Transition zones } \\
\text { Overburden thickness }\end{array}$ & Northern West Virginia & \\
\hline $\begin{array}{c}\text { Riefenberg } \\
1996\end{array}$ & $\begin{array}{l}\text { CMRR } \\
\text { Mudstone thickness } \\
\text { Vertical Stress } \\
\text { Depth to seam } \\
\text { Floor Rating }\end{array}$ & & Equal weights \\
\hline $\begin{array}{c}\text { Sames } \\
1995\end{array}$ & $\begin{array}{l}\text { Overburden thickness } \\
\text { Roof strength } \\
\text { Floor strength }\end{array}$ & $\begin{array}{l}\text { Pocahontas No. 4, WV } \\
\text { Pocahontas No. 3, VA }\end{array}$ & Equal weights \\
\hline $\begin{array}{c}\text { Riefenberg } \\
1994\end{array}$ & $\begin{array}{l}\text { CMRR } \\
\text { Stress interaction between } \\
\text { two seams }\end{array}$ & & Equal weights \\
\hline
\end{tabular}




\section{Chapter 7. Summary and Conclusions}

\subsection{Summary}

Stability (or hazard) mapping is conducted in many underground mines to analyze the roof stability prior to or during the mining operation. A mine map with accurate stability analysis helps mining engineers to predict the potential weak roof areas and to design more productive, safer mines. However, it is well known that mine stability is impacted by multiple parameters such as geology properties, geologic structures, in-situ stresses and geo-mechanical stresses. Therefore, to correctly evaluate the stability of a mine, all critical influence factors should be identified and analyzed. Due to the diversity of the stability influence factors and the complexity of the geologic conditions at a particular mine, current hazard mapping methods have been found to have many practical limitations. For instances, many tedious tasks such as the data collecting and analyzing have to be done either manually, by using separate application tools from different sources. Also some of the numerical modeling techniques used for stress analysis are either less accurate or too complicated to apply by practicing engineers. As a response to the increasing demand for a multiple-factor stability analysis, an integrated stability mapping system has been developed with the goal to provide practicing mining engineering with a comprehensive, flexible and technologically advanced solution.

This dissertation presents the system design and implementation of an integrated stability mapping system into a full-featured, CAD based program with a broad stability analysis capability for a large mine area. This integrated stability mapping system includes: automatic data processing for various influence factors, advanced stress analysis modeling, various influence factor and stability index calculations, and multiple- 
parameter stability index composting and mapping. By applying the integrated stability mapping system, a typical stability mapping analysis goes through the following procedures:

- Define and input the critical geologic, structural and stress factors which have the most influences on the mine stability.

- Calculate a numeric grid for each critical factor.

- Determine an individual index for each critical factor (transformation)

- Compose the overall stability index (ex. Weighting the individual indices )

- Plot and analyze the overall stability index on the mine map.

- Re-evaluate critical factors and weightings as necessary.

The main goal of the modules developed in the integrated stability mapping system is to facilitate all of these steps by providing and integrating the appropriate techniques.

The first step in the development of the integrated stability mapping system was to classify the potential influence factors on mine stability. Three main categories were developed according to the characteristics of the influence factors namely geologic properties, structural features and geo-mechanical stresses. The geologic properties refer to the general geologic conditions such as layer thickness, chemical composition, lithologic variations, etc. The structural features describe the geologic and topographic structures both on the surface and underground such as stream valleys, sandstone channels, faults, etc. The geo-mechanical stress may include overburden stress, interseam stress, and horizontal stress. For a specific mine, some of these factors will have a 
more critical impact on mine stability than others, and these critical influence factors should be the main input parameters for the mine stability analysis.

For these potential stability influence factors, a number of techniques have been developed to calculate their quantitative values. In general, SurvCADD mining modules which are built into the AutoCAD environment can be used to grid and contour the general geology properties from exploration drillhole logs. Actually, many mines already have this type of SurvCADD information readily available. Also, some basic data gridding routines which can be used for simple geologic input are included with the structural modules of the stability mapping system for mines without SurvCADD access.

For the structure features, the input information will be provided with different graphic representation forms such as standard AutoCAD objects:

- Points - Specify the values of a certain factor at random locations.

- Lines/Polyline - Represent the location of linear structural features such as: faults, stream valleys, cutter roof, etc.

- Contour Polylines - Provide the values of a geologic property (thickness, overburden, etc.) along a 3-dimensional Polyline.

- Polygons - Represent the location of the area-bounded features such as: sandstone channels, stream valleys, ponds, gob areas, etc.

For each specific type of structural input, there is an independent application module which takes the appropriate input information and calculates the values for the factor over the specified grid using user specified influence areas and defined algorithms.

For the geo-mechanical stress input, the stress analysis in the stability mapping system adopts the methodology of the LaModel stress calculation, because it has been 
proven to be a rather successful numerical technique in analyzing the stresses and displacements at the seam level. LaModel stress calculation has been able to be calculated directly from inside the stability system. Modules in the stability mapping system also allow the user to open a LaModel output file and import any of the enclosed output values as a grid in the stability mapping system. This includes such values as: convergence, overburden stress, total vertical stress, multiple-seam stress, pillar safety factor, subsidence, horizontal strain, etc.

For a simple topographic stress analysis, the overburden stress calculation implemented the same algorithm as LaModel has been built directly into the stability mapping module, and the overburden stresses can be calculated and gridded completely within the AutoCAD interface. Also, the integrated stability mapping system has modules for automatically generating the topographic and mine grids to be used as input to the LaModel stress analysis.

Once all of the critical influence factors have been input, gridded and saved in the database, the major effort in data input for the stability mapping system is completed and the core grid database has been created. The next step is to calculate individual stability indices by applying the appropriate transformation function to each factor. In order to eventually combine all of the individual critical factors into one overall stability index, each of the individual factors has to first be normalized into the same numeric range ( 0 100 with larger values typically representing less stable conditions) by using an appropriate transformation function. The transformation functions presently include: Linear, Step, and Exponential. Also, complex user-defined behaviors can be achieved with operation in the grid utility module 
After all of the individual indices have been determined using the appropriate transformations, the next step is to calculate an overall stability index by combining the individual values. As discussed above, the overall mine stability can be related to many geologic, structural and/or stress factors. In the general case, certain factors may have more influence on the final stability than others; therefore, the final calculation of the overall stability index module implemented a straightforward weighting process which allows the user to apply various weights to each individual factor index in order to calculate the final overall stability index. For complex, user-customized calculations of the stability index, as demanded in certain cases, can be accomplished by manipulating the individual index grids with the comprehensive operation in the grid utility module.

After the final stability index is calculated, it is desirable to plot the results or any grid on the mine map for analysis. For the optimum analysis of the stability index, it can be plotted as: points, contour lines, or a colored grid with lines or solids (see the figures for the case study in Chapter 6). Of course, the calculated stability index should ultimately be calibrated/validated against field observations or measurements, and any necessary adjustments to the critical factor scaling or weighting needed to optimize the accuracy of the final stability index should be made. By calibrating the stability index with field data, the user can gain a certain degree of confidence in the system output.

The actual stability program is written in Microsoft Visual C++ plus AutoCAD ObjectARX extensions. The whole system consists of a number of function modules which reside in the AutoCAD environment as add-on commands when the application is loaded into AutoCAD memory space. Typically, an influence factor process module takes input data from specific layers of mine map, calculates the numerical values for this 
property based on the control parameters and selected algorithm, and saves the results (for example, property's values, individual stability indices, stresses and/or displacements) into an output grid file. Generally, the input layers of a mine map should contain the 3D graphical representations of the desired influence factor. Their geometries usually are of point, line, polyline (polygon), and contour line. After these input data are read into memory, the module then processes the inputs with the appropriate algorithm. The resultant output grid files form the basic stability data base. These grid files may then be used as input files for other stability mapping modules to process further (calculation, display and plotting). A consistent grid file structure (see Appendix A) is used for all the stability modules, and the file structure is also compatible with the SurvCADD grid file format. This allows to fully utilizing the advanced mining modules in SurvCADD and the enormous geologic information already available in SurvCADD format.

After completing programming of the code for the stability mapping system and initial checking of the code for basic logic errors, two case studies were performed to demonstrate the utility and application of the system. The first study included two critical influence factors namely the overburden stress and a fracture zone under a stream valley, whereas three factors were incorporated in the second case study: the sandstone lenses thickness in the roof, the interval between the sandstone lenses and the seam, and the overburden stress. Although both case studies are only simple demonstration and only a limited number of influence factors were considered in the final stability index calculation, the resultant stability index maps have provided examples how multiple factors could interact with each other to influence the overall mine stability. With more site specific information and optimized transformations and weighting processes, the 
developed stability mapping would help mining engineers to obtain sufficient foreknowledge of the roof stability distribution, and to identify the potential unstable mining area.

\subsection{Conclusions}

Based on the experience gained in performing the system analysis and case studies in this dissertation, it appears that the integrated stability mapping system can be a convenient and comprehensive software package for the practicing mine engineer to utilize in conducting complex stability mapping activities. Geology factors such as strata thickness, strength, and composition; structural factors such as faults, stream valleys and sandstone channels; and geo-mechanical factors such as overburden stress, multiple-seam stress and subsidence-based strain can be easily integrated into one single stability factor for a mine property. Embracing the advanced computer aided design technology and the realistic boundary element numerical stress solution, the stability mapping system has been developed upon the popular AutoCAD drawing software and SurvCADD mine planning software, and the consistent, integrated graphical interface and data file structure greatly reduces the time and effort required for a new user to produce a functional stability map. The system mapping features also provides a full range of capabilities for plotting and analyzing the calculated stability indices.

It is anticipated that through application of the integrated stability mapping system, mine engineers can obtain foreknowledge and a better understanding of unstable mining areas. With this enhanced knowledge, the mine and support design can then be optimized in order to develop safer and more productive mines. 


\subsection{Ideas for Additional Research}

While implementing the stability mapping system and applying the system to the case studies presented in this dissertation, numerous ideas for additional research were conceived. Some of these ideas were immediately implemented and included in this dissertation. For instance, the idea of including the horizontal stress impact arose naturally when reviewing the stress influence and implementing the LaModel overburden stress calculation. It has been well understood that the direction of the maximum horizontal stress is very important to ground control problem and the orientation of the maximum horizontal stress in the eastern United States has been fairly well defined and its influence on roof stability has also been thoroughly studied by many researchers over years. The seasonal feature demanded immediate implementation into the stability mapping system. Similarly, the need for the automatic seam grid generation arose in examining the performance of LaModel against the actual irregular mine pillar layouts. A full featured automatic seam grid generation module was implemented with faster algorithms and an AutoCAD visual interface. In one comparative study, the new module has reduced the calculation time from hours to several seconds.

Some of the ideas conceived while performing the work in this dissertation deserve additional research. The impact of the maximum horizontal stress direction on mine stability has been well documented, but the other important aspects of the horizontal stress, the magnitude and the magnitude variation, are not well understood. In the past, researchers have been trying to link the overburden depth and the increase in the magnitude of the horizontal stress by some empirical equations from statistical analysis. 
However the correlation is weak and how the magnitude affects the final roof stability locally and regionally remains uncertain and controversial.

Also, although the modules developed in the stability mapping system have cover the most aspects of the stability mapping requirements, new needs could arise when applying to the actual mine situations. Thanks to the open structure of the stability system inherited from AutoCAD, any new features can be incorporated in the system easily and smoothly.

Taking the known geology and converting it to an engineering input for roof support design has always been problematic. One widely used method/tool for classifying the geology for engineering design and roof support selection is the Coal Mine Roof Rating system (Molinda and Mark, 1996; Molinda and Mark, 1994). The Coal Mine Roof Rating (CMRR) is a geologic classification system that was specifically developed for bedded coal measure rocks. It concentrates on the discontinuities in the bolted interval above the coal seam, and uses simple field tests and observations to calculate a rating number between one and one hundred which correlates to the engineering "strength" of the mine roof. It can be determined directly from geologic cores, if necessary. For integrating CMRR with the stability mapping program, the capability of calculating the Coal Mine Roof Rating from the appropriate input geology (and additional user input) is needed. This will enable the CMRR for the mine to be overlain on the entire modeled mine plan providing a "universal" industry-wide index of the mine roof strength (Molinda and Mark, 1996; Molinda and Mark, 1994). This index in combination with other geology and/or stress influence factors would enable the 
program to implement the NIOSH design equations and recommend roof support design parameters in the future development.

Although two simple case studies have been performed in this dissertation, more extensive validations and evaluations with actual field data are necessary to verify the utility and accuracy of the system. Through more through evaluation process, further refinements can be made to the user-interface, and additional practical transformation functions and weighting ratios can be developed and documented. At these additional field verification sites, the necessary geologic and mining data would be collected along with the mine specific stability criteria. This collected data can then be used to calculate a stability index and to determine roof support recommendations. At the mine sites, underground visual observation would be collected on: the geology, the roof support design, the apparent stress (through stress mapping) and the observed stability. These underground observations can then be correlated with the calculated stability index and roof support recommendations. Based on this correlation and actual field application of the program, it is expected that various enhancements may be incorporated into the initial system and that the original transformation approaches and weighting factors may be adjusted to more accurate values. 


\section{REFERENCES}

Akinkugbe, O. and K. Heasley. 2004. The new Two-Dimension LaModel Program. Proceedings of the 23rd International Conference on Ground Control in Mining. West Virginia University, Morgantown, W.Va., pp. 146-153.

Autodesk. 2001, 2003. ObjectARX References. Autodesk Inc. San Rafael, CA

Berry, D. and T. Sales. 1962. "An Elastic Treatment of Ground Movement Due to Mining - Part II." Transversely Isotropic Ground. Journal of Mechanics and Physics of Solids, Vol. 9, pp. 52-62.

Canbulat I., L. Munsamy and D. Minney. 2004. A Risk Assessment Tool for Open Cast Mining in South Africa. Proceedings of the 23rd International Conference on Ground Control in Mining. West Virginia University, Morgantown, W.Va., pp. 205-209.

Carlson Software. 2001. SurvCADD - CES. Carlson Software, Maysville, KY.

Chekan, G. and J. Listak. 1994. Design Practices for Multiple-Seam Room-and-Pillar Mines. USBM IC 9403, 44 pp.

Chekan, G. and J. Listak. 1993. Design Practices for Multiple-Seam Longwall Mines. USBM IC 9360, $33 \mathrm{pp}$.

Crouch, S. and A. Satrfield. 1983. Boundary Element Methods in Solid Mechanics, George Allen \& Unwin (Publishers) Ltd, London, UK

Dolinar, D. 2001. Analysis of Ground Fall Injury Statistics for Underground Coal Mines 1995 to 1999. Internal memo report. 19pp.

Dolinar D. 2003. Variation of Horizontal Stresses and Strains in Mines in Bedded Deposits in the Eastern and Midwestern United States. Proceedings of the 22nd International Conference on Ground Control in Mining. West Virginia University, Morgantown, W.Va., pp. 178-185.

Finfinger, G., S. Peng, Q. Gu, G. Wilson and B. Thomas. 2000. An Approach to Identify Geologic Properties from Roof Bolter Drilling Parameters. Proceedings of the 19th International Conference on Ground Control in Mining. West Virginia University, Morgantown, W.Va., pp. 1-11.

Gadde, M. and S. Peng. 2004. Effect of in Situ Stresses on the Stability of Coal Mine Development Workings, Proceedings of the 23rd International Conference on Ground Control in Mining. West Virginia University, Morgantown, W.Va., pp. 92-102. 
Hanna, K., K. Haramy and D. Conover. 1986. Effect of High Horizontal Stress on Coal Mine Entry Intersection Stability. Proceedings of the 5th International Conference on Ground Control in Mining. West Virginia University, Morgantown, W.Va., pp. 167-181.

Heasley, K, and Q. Wang. 2001. Integrated Stability Mapping System for Mines, Research proposal submitted to National Institute for Occupational Safety and Health, Morgantown, WV.

Heasley, K. J. Ellenberger. And P. Jeran. 2001. An Analysis of Rock Failure around a Deep Longwall Using Microseismics. Proceedings of the 20th International Conference on Ground Control in Mining. West Virginia University, Morgantown, W.Va., pp. 280286.

Heasley, K, and Z. Agioutantis. 2001. "LAMODEL - A Boundary Element Program for Coal Mine Design." Proceedings of the 10th International Conference on Computer Methods and Advances in Geomechanics, Tucson, Arizona, pp. 1679-1682.

Heasley, K. and T. Barton. 1999. "Coal Mine Subsidence Prediction Using a BoundaryElement Program." Transactions of the Society of Mining, Metallurgy and Exploration, Inc., Vol. 306, pp. 99-104.

Heasley, K, and G Chekan. 1998. "Practical Stress Modeling for Mine Planning." Proceedings of 17th International Conference on Ground Control in Mining, Morgantown, WV, pp.129-137.

Heasley, K. 1998. Numerical Modeling of Coal Mines with a Laminated DisplacementDiscontinuity Code. Ph.D. Dissertation, Colorado School of Mines, Golden, CO, 187 pp.

Heasley, K and J. Zelanko. 1992. "Pillar Design in Bump-Prone Ground Using Numerical Models with Energy Calculations." Proceedings of the Workshop on Coal Pillar Mechanics and Design. USBM IC 9315, p. 50-60.

Heasley, K., Z. Agioutantis, and Q. Wang. 2003. "Automatic Grid Generation Allows Faster Analysis of Coal Mines", Proceedings, SME Annual Meeting, Cincinnati, Ohio, February 2003.

Hill, J. 1988. The Influence of Stream Valleys on Coal Mine Ground Control. Proceedings of the 7th International Conference on Ground Control in Mining. West Virginia University, Morgantown, W.Va., pp. 247-258.

Jiang, Y. and B. Wells. 1998. Analysis of Geologic and Geotechnical Conditions and Their Effects on Longwall Mining to Optimize Mine Planning at Shoal Creek Mine. Proceedings of the 17th International Conference on Ground Control in Mining. West Virginia University, Morgantown, W.Va., pp. 54-62. 
Jones, T. and A. Dennis. 1986. Analysis of Small-scale Thrust Faults and their Effect on Coal Mining in Southern West Virginia. Proceedings of the 5th International Conference on Ground Control in Mining. West Virginia University, Morgantown, W.Va., pp. 183190.

Joseph O'Rourke. 1998. Computational Geometry in C. Cambridge University Press

Keim, K. Scott and M. S. Miller. 1999. Case Study Evaluation of Geological Influences Impacting Mining Conditions as a West Virginia Longwall Mine. International Journal of Coal Geology. Vol 44. pp. 51-71.

Kripakov, N. and L. Rockwell. 1989. "Application of Numerical Modeling Techniques for Gate Road Design in Bump Prone Mines." Proceedings of Longwall USA, Pittsburgh, PA, p. 87-99.

Kripakov, N., L. Beckett, D. Donato and J. Durr. 1988. Computer Assisted Mine Design Procedures for Longwall Mining. USBM RI 9172

Maleki, H. 1990. "Development of Modeling Procedures for Coal Mine Stability Evaluation." Proceedings of the 31st U.S. Rock Mechanics Symposium. Rotterdam: Balkema, p. 85-92.

Mark, C. 1987. Analysis of Longwall Pillar Stability. Ph.D. dissertation, The Pennsylvania State University, University Park, PA.

Mark, C., G. Molinda and D. Dolinar. 2001. Analysis of Roof Bolt Systems. Proceedings of the 20th International Conference on Ground Control in Mining. West Virginia University, Morgantown, W.Va., pp. 218-225.

Mark, C. and F. Chase. 1997. "Analysis of Retreat Mining Pillar Stability (ARMPS)." Proceedings of New Technology for Ground Control in Retreat Mining. NIOSH IC 9446, p. 17-34.

Molinda, G. and C. Mark. 1996. Rating the Strength of Coal Mine Roof Rocks. USBM IC 9444, 36 pp.

Molinda, G. and C. Mark. 1994. Coal Mine Roof Rating (CMRR): A Practical Rock Mass Rating for Coal Mines. USBM IC 9387, 83 pp.

Molinda, G., K. Heasley, D. Oyler and J. Jones. 1991. Effects of Surface Topography on the Stability of Coal Mine Openings. Proceedings of the 10th International Conference on Ground Control in Mining. West Virginia University, Morgantown, W.Va., pp. 151-160.

Newman, D., J. DeCinque and A. Dafferner. 2001. The Integration of Geology and Engineering in Ground Control and Mine Planning for a Multi-Level Underground 
Limestone Quarry. Proceedings of the 20th International Conference on Ground Control in Mining. West Virginia University, Morgantown, W.Va., pp. 129-136.

Newman, D., Z. Agioutantis and M. Karmis. 2001. SDPS for Windows: An integrated Approach to Ground Deformation Prediction. Proceedings of the 20th International Conference on Ground Control in Mining. West Virginia University, Morgantown, W.Va., pp. 157-162.

Peters, D. and R. Speirer. 1986. A Case History of Computer-aided Lineament Analysis for Ground Control Planning. Proceedings of the 5th International Conference on Ground Control in Mining. West Virginia University, Morgantown, W.Va., pp. 191-205.

Riefenberg, J. 1996. Geostatistical Methods for Hazard Assessment and Site Characterization in Mining. Proceedings of the 15th International Conference on Ground Control in Mining. Colorado School of Mines, Golden, CO, pp. 671-683.

Riefenberg, J. 1994. Hazard Mapping Combining Geostatistical Modeling of Coal Mine Roof Quality Ratings with Numerical Modeling of Stress Data. Proceedings of the 13th International Conference on Ground Control in Mining. West Virginia University, Morgantown, W.Va., pp. 261-268.

Rusnak, J. 1998. Application of the Coal Mine Roof Rating, Derived from Drill Core, in the Roof Support Design of a Coal Belt Conveyor Tunnel. Proceedings of the 17th International Conference on Ground Control in Mining. West Virginia University, Morgantown, W.Va., pp. 221-230.

Sames, Gary. 1995. Bump Hazard Criteria Derived from Basic Geologic Data. Proceedings: Mechanics and Mitigation of Violent Failure in Coal and Hard-Rock Mines. Special Publication 01-95. US Department of the Interior, Bureau of Mines. pp. 69-89.

Sinha, K., 1979. Displacement Discontinuity Technique for Analyzing Stress and Displacements Due to Mining in Seam Deposits. Ph.D. Dissertation, University of Minnesota, MN, $311 \mathrm{pp}$.

Stankus, J., Y. Wang and J. McCaffrey. 2001. Roof Instability Rating (RIR) System and Its Application at Enlow Fork Mine. Proceedings of the 20th International Conference on Ground Control in Mining. West Virginia University, Morgantown, W.Va., pp. 327-336.

Stankus, J. and Y. Wang. 1999. Localized Horizontal Stress and its Effect, Proceedings of the 18th International Conference on Ground Control in Mining. West Virginia University, Morgantown, W.Va., pp. 309-314.

Stolarczyk, L., S. Peng and Y. Luo. 2003. Imaging Ahead of Mining with Radio Imaging Method(RIM-IV) Instrumentation and Three-Dimensional Tomography Software. Proceedings of the 22nd International Conference on Ground Control in Mining. West Virginia University, Morgantown, W.Va., pp. 278-282. 
$\mathrm{Su}, \mathrm{D} .$, E. Thomas and G. Hasenfus. 1999. Roof Geology Mapping in Underground Coal Mines, Proceedings of the 18th International Conference on Ground Control in Mining. West Virginia University, Morgantown, W.Va., pp. 40-45.

Vandergrift, T., T. Ross, C. Hawley and J. Rusnak. 2000. Mine Planning for Longwall and Pillar Retreat Panels Subject to Seam Interaction Effects. Proceedings of the 19th International Conference on Ground Control in Mining. West Virginia University, Morgantown, W.Va., pp. 204-212.

Watts, M., Y. Wang and J. Stankus. 2000. Roof Control Analysis in North River Mine. Proceedings of the 19th International Conference on Ground Control in Mining. West Virginia University, Morgantown, W.Va., pp. 332-340.

Westman, E. 2003. Recent Developments in the Use of Seismic Tomography in Longwall Mines. Proceedings of the 22nd International Conference on Ground Control in Mining. West Virginia University, Morgantown, W.Va., pp. 278-282.

Zipf, R. and K. Heasley. 1990. Decreasing Bump Risk through Optimal Cut Sequencing with a Non-Linear Boundary Element Program. Proceedings of the 31st US Rock Mechanics Symposium. Rotterdam: Balkema, pp. 947-954.

Zipf, R., 1992a, Mulsim/NL Application and Practitioner's Manual, BuMines IC 9322.

Zipf, R., 1992b, Mulsim/NL Application Theoretical and Programmer's Manual, BuMines IC 9321. 


\section{Appendix A. Grid File}

This appendix contains a description and example of the system grid files that are created and/or used by the stability mapping system. These grid files form a consistent base such that the grid files can be read, written and manipulated by all system modules at any stage of the analysis process. Following is an example of a typical grid file:

587842.590000

1216893.660000

590142.590000

1220893.660000

400

230

861.710026

861.205489

860.662810

860.081383

Overburden Stresses

SMAP Stability Application

The first six lines in the grid file define the location of the grid:

Line 1 is the lower left $\mathrm{Y}$ coordinate.

Line 2 is the lower left $\mathrm{X}$ coordinate.

Line 3 is the upper right $\mathrm{Y}$ coordinate. 
Line 4 is the upper right $\mathrm{X}$ coordinate.

Line 5 is the $\mathrm{X}$ direction grid resolution (number of elements).

Line 6 is the $\mathrm{Y}$ direction grid resolution (number of elements).

The rest of the lines are the $\mathrm{Z}$ values of the grid elements starting from the lower left moving in the left to right direction and ending at the upper right. In order to compatible with SurvCADD grid file, an extra element is appended into each direction, therefore, the total lines of the $\mathrm{Z}$ values will be:

$(\mathrm{X}$ direction grid resolution +1$) *(\mathrm{Y}$ direction grid resolution +1$)$

After the all $\mathrm{Z}$ values in the file is one line description of the grid data. That was usually input as a title by the user when building the grid using the stability mapping modules.

The last line with the text SMAP Stability Application implies that this grid file was created by using the stability mapping modules. This line is used by the system to distinguish the file from the SurvCADD generated grid files.

The main difference between SurvCADD generated grid file and the stability system grid file is that the grid file created by SurvCADD stores the $\mathrm{Z}$ values of each grid intersections instead of the center of the grid elements as in the stability system grid. When a stability system module is reading in a SurvCADD grid file, a necessary calculation will be done internally to convert its format to the stability system format. 


\section{Appendix B. Menu System and Principal Input Forms}

This appendix contains screen shots and brief explanations of the input forms of the stability mapping system. In an effort to simplify the structure and utility of the application, the functional modules in the system are designated to be independent and a global system menu is used to access these modules.

\section{B.1 System Menu}

A user accesses the modules of the stability mapping system using a pull-down menu in AutoCAD application. When the application file stability.arx, a standard AutoCAD ObjectARX application file, is loaded into the AutoCAD (generally using the standard AutoCAD command appload, see Figure B.1), a new pull-down menu entry will be automatically added into the system menu bar with a title "Stability Mapping".

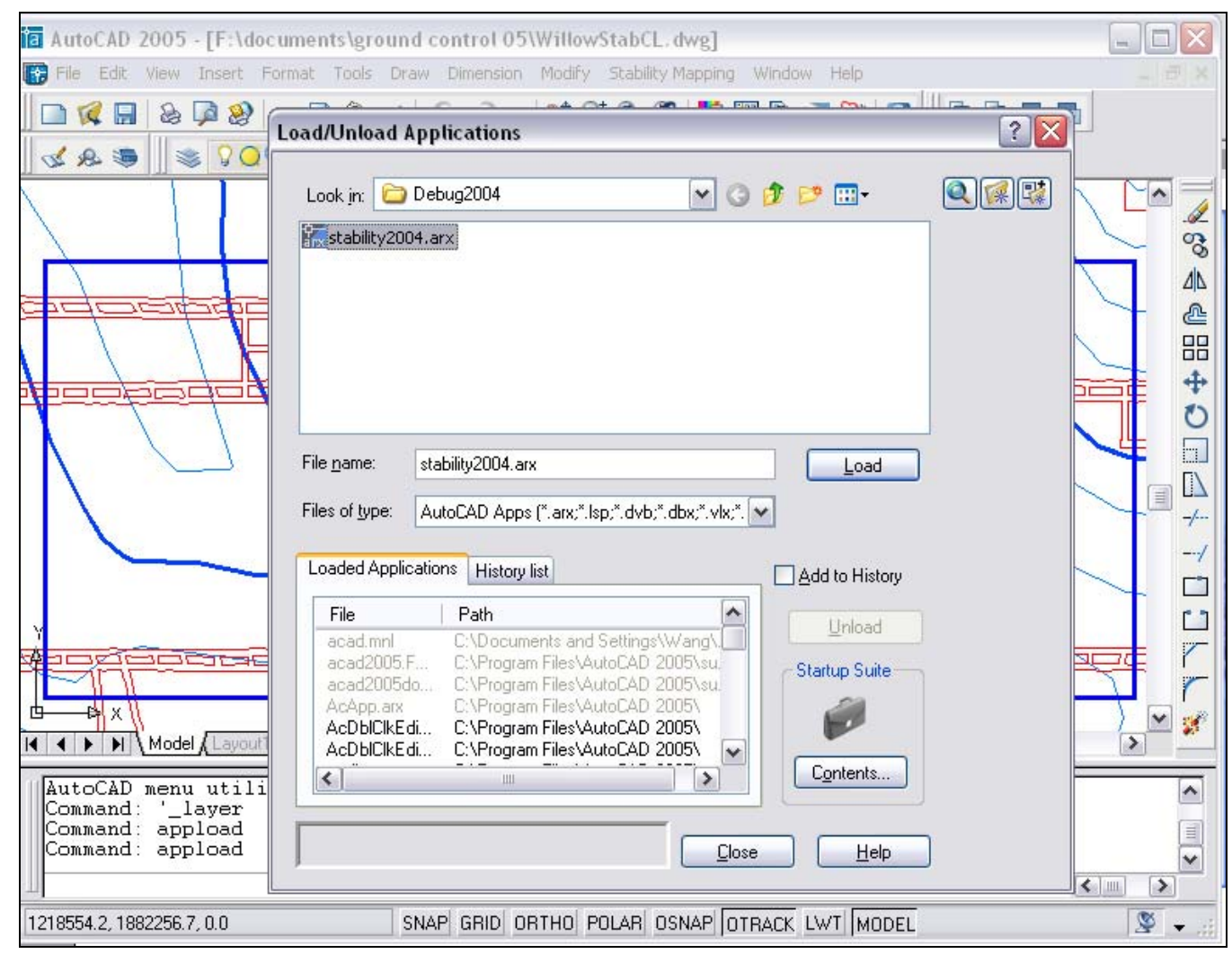

Figure B.1 Load stability application 
Under this menu entry, there are a lot of menu items which provide access to all of the functionality of the stability mapping system (see Figure B.2). The menu items are arranged by groups. A brief description of the menu items can be found in Table B.1.

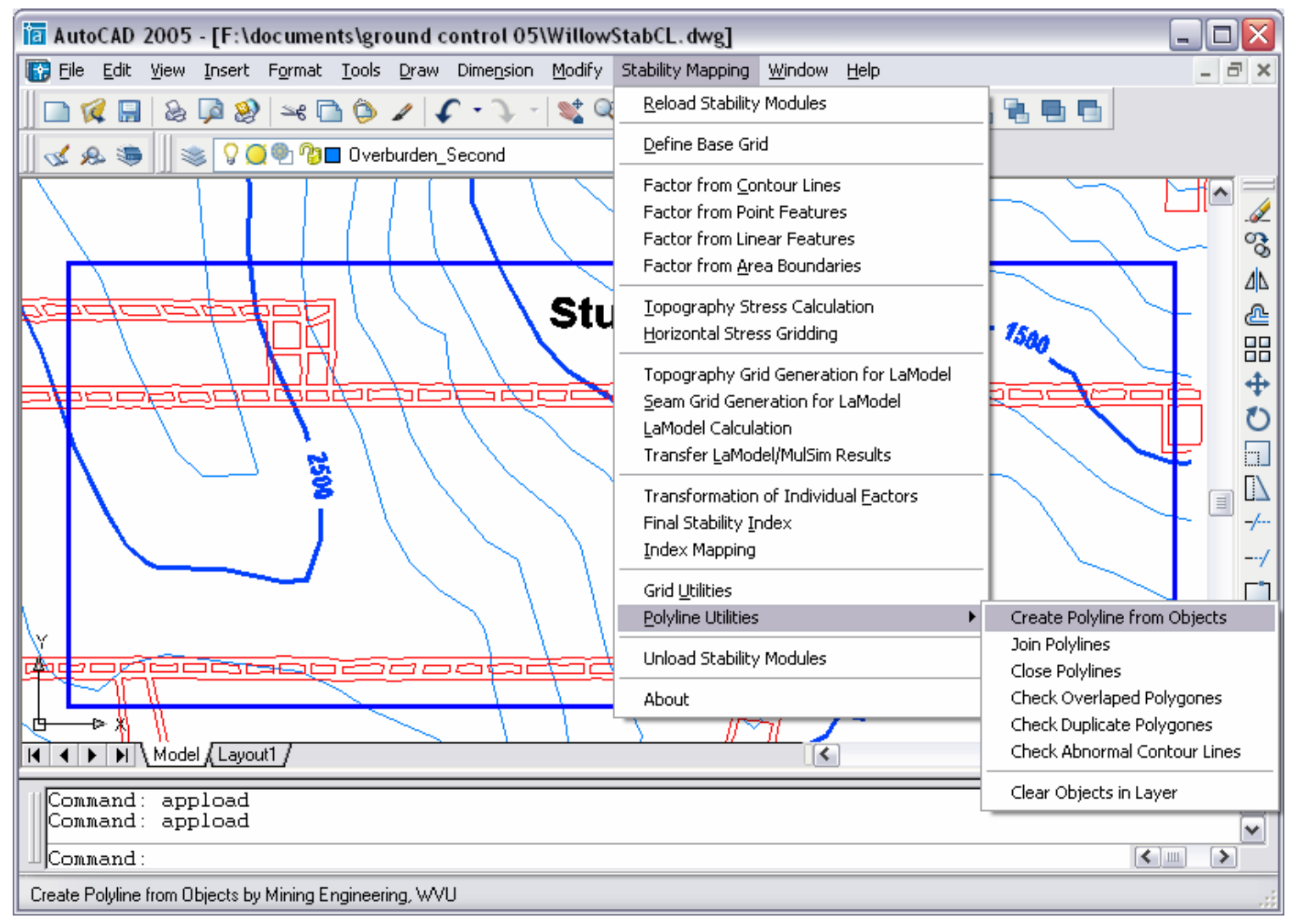

Figure B.2 Stability mapping menu system

Table B.0-1 Description of Menu Items

\begin{tabular}{|c|c|c|}
\hline \multicolumn{2}{|c|}{ Menu Items (in order) } & Function Description \\
\hline \multicolumn{2}{|c|}{ Reload Stability Modules } & $\begin{array}{l}\text { Reload the stability mapping application into the } \\
\text { AutoCAD environment }\end{array}$ \\
\hline \multicolumn{2}{|c|}{ Define Base Grid } & $\begin{array}{l}\text { Defines a base grid as a default grid setting for } \\
\text { the current mine map. The settings will be saved } \\
\text { and can be used by other modules. }\end{array}$ \\
\hline $\begin{array}{l}\text { Geological } \\
\text { Gridding }\end{array}$ & Contour Lines & Generates grid files from contour lines \\
\hline
\end{tabular}




\begin{tabular}{|c|c|c|}
\hline \multirow{3}{*}{$\begin{array}{l}\text { Geological } \\
\text { Gridding } \\
\text { Modules }\end{array}$} & Point Features & $\begin{array}{l}\text { Generates grid files from point-type geological } \\
\text { features such as drill holes. }\end{array}$ \\
\hline & Linear Features & $\begin{array}{l}\text { Generates grid files from linear geological } \\
\text { features such as faults. }\end{array}$ \\
\hline & Area Boundaries & $\begin{array}{l}\text { Generates grid files from bounded areas such as } \\
\text { stream valleys and ponds. }\end{array}$ \\
\hline \multirow{2}{*}{$\begin{array}{l}\text { Stress } \\
\text { Modules }\end{array}$} & $\begin{array}{l}\text { Topography Stress } \\
\text { Calculation }\end{array}$ & $\begin{array}{l}\text { Calculates the overburden stress using } \\
\text { overburden thickness grid. }\end{array}$ \\
\hline & $\begin{array}{l}\text { Horizontal Stress } \\
\text { Gridding }\end{array}$ & $\begin{array}{l}\text { Calculates the influence factor for the orientation } \\
\text { effect of the maximum horizontal stress on mine } \\
\text { entries. }\end{array}$ \\
\hline \multirow{4}{*}{$\begin{array}{l}\text { LaModel } \\
\text { Calculations } \\
\text { and } \\
\text { Enhancements }\end{array}$} & $\begin{array}{l}\text { Topography Grid } \\
\text { Generation }\end{array}$ & $\begin{array}{l}\text { Automatic generates topography grid file for } \\
\text { LaModel analysis. }\end{array}$ \\
\hline & $\begin{array}{l}\text { Seam Grid } \\
\text { Generation }\end{array}$ & $\begin{array}{l}\text { Automatic generates seam grid definitions for } \\
\text { LaModel analysis }\end{array}$ \\
\hline & $\begin{array}{l}\text { LaModel } \\
\text { Calculation }\end{array}$ & Applies standard LaModel Stress Calculation \\
\hline & $\begin{array}{l}\text { LaModel/MulSim } \\
\text { Result Transfer }\end{array}$ & $\begin{array}{l}\text { Transfers the results of LaModel and/or MulSim } \\
\text { into grid files of the Stability Mapping System }\end{array}$ \\
\hline \multirow{3}{*}{$\begin{array}{l}\text { Index } \\
\text { Mapping } \\
\text { Modules }\end{array}$} & $\begin{array}{l}\text { Transformation of } \\
\text { Individual Factors }\end{array}$ & $\begin{array}{l}\text { Generates an individual stability index for a } \\
\text { selected influence factor }\end{array}$ \\
\hline & Final Stability Index & $\begin{array}{l}\text { Creates composite stability index using a } \\
\text { weighted average method. }\end{array}$ \\
\hline & Index Mapping & Maps the stability index grid over entry layout. \\
\hline \multirow{2}{*}{ Help Modules } & Grid Utilities & Views, plots and manages grid files. \\
\hline & Polyline Utilities & $\begin{array}{l}\text { A group of commands to join, close, examine, } \\
\text { and create polygons/polylines in mine maps. }\end{array}$ \\
\hline \multicolumn{2}{|c|}{ Unload Stability Modules } & $\begin{array}{l}\text { Unload the stability mapping application from } \\
\text { AutoCAD system. }\end{array}$ \\
\hline \multicolumn{2}{|l|}{ About } & About dialog, contact information \\
\hline
\end{tabular}




\section{B.2 Principal forms of the stability mapping system}

This section shows the user interfaces/forms of the principal stability mapping modules and explains their main input parameters. It should be noted that many of the user forms integrated various interactive functions to help user to optimize the input data on the fly instead of just an input-run-output type calculation.

\section{- Grid definition form}

Figure B.3 shows the standard form for grid definition. This form is also embedded into other stability mapping modules which require parameters of the grid dimensions.

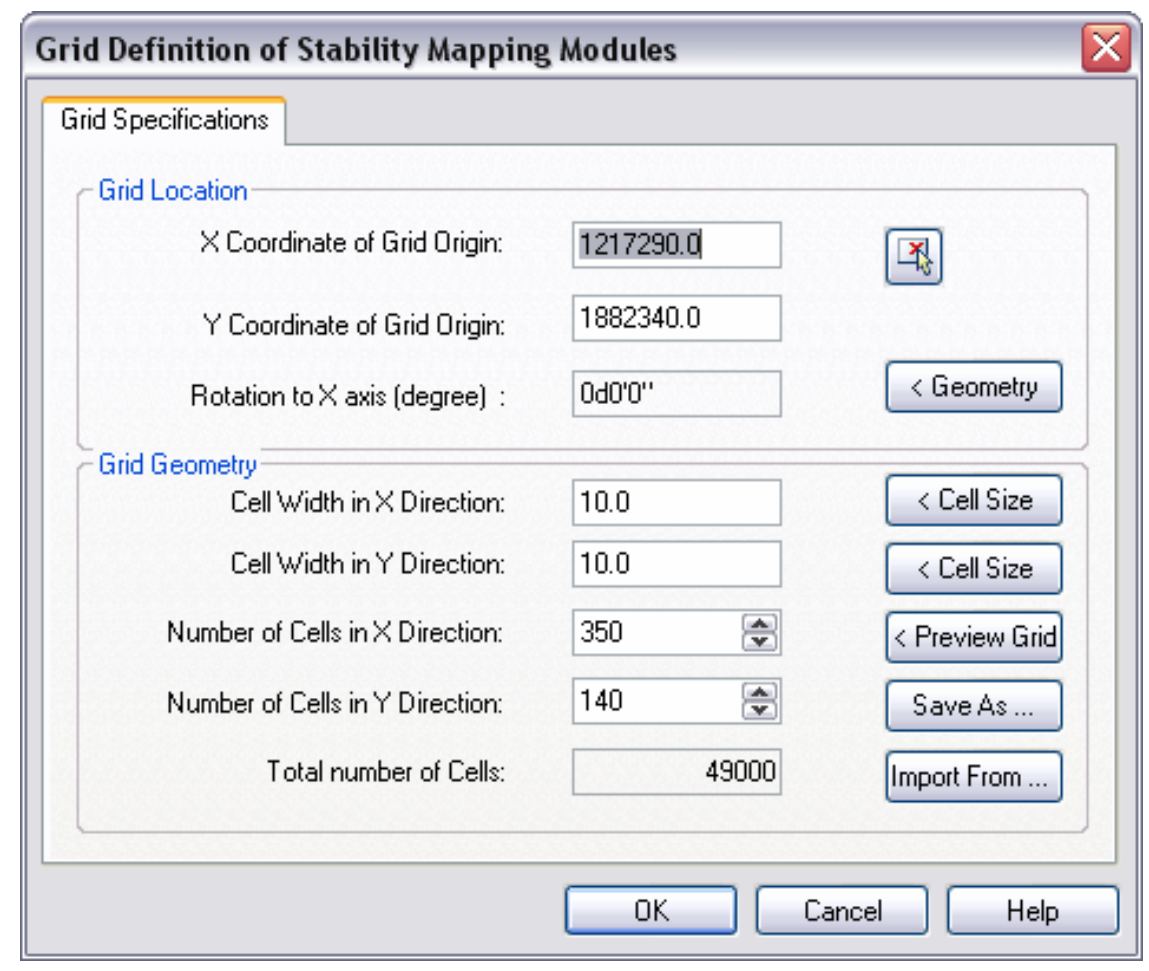

Figure B.3 Grid definition form

. In this form, the parameters are set as the default base grid, and these parameters can be changed in the input form of other individual modules if needed. This form includes the parameters necessary to define a grid such as grid location and dimension. 
Most of these parameters can also be specified interactively in the AutoCAD model view. The grid definition can be saved to a grid file or imported from an existing grid file. The resultant grid can be previewed in the AutoCAD model view. Typically, when the form is closed by clicking the $\boldsymbol{O} \boldsymbol{K}$ button, the parameters will be set as the default values in following sessions.

\section{- Grid generation for contour form}

Figure B.4 is the input form to create an influence grid for geologic properties from contour lines on the mine map. There are two tabs on this form. The first one is the standard grid definition form and the second tab is a form with specific inputs for gridding contour lines.

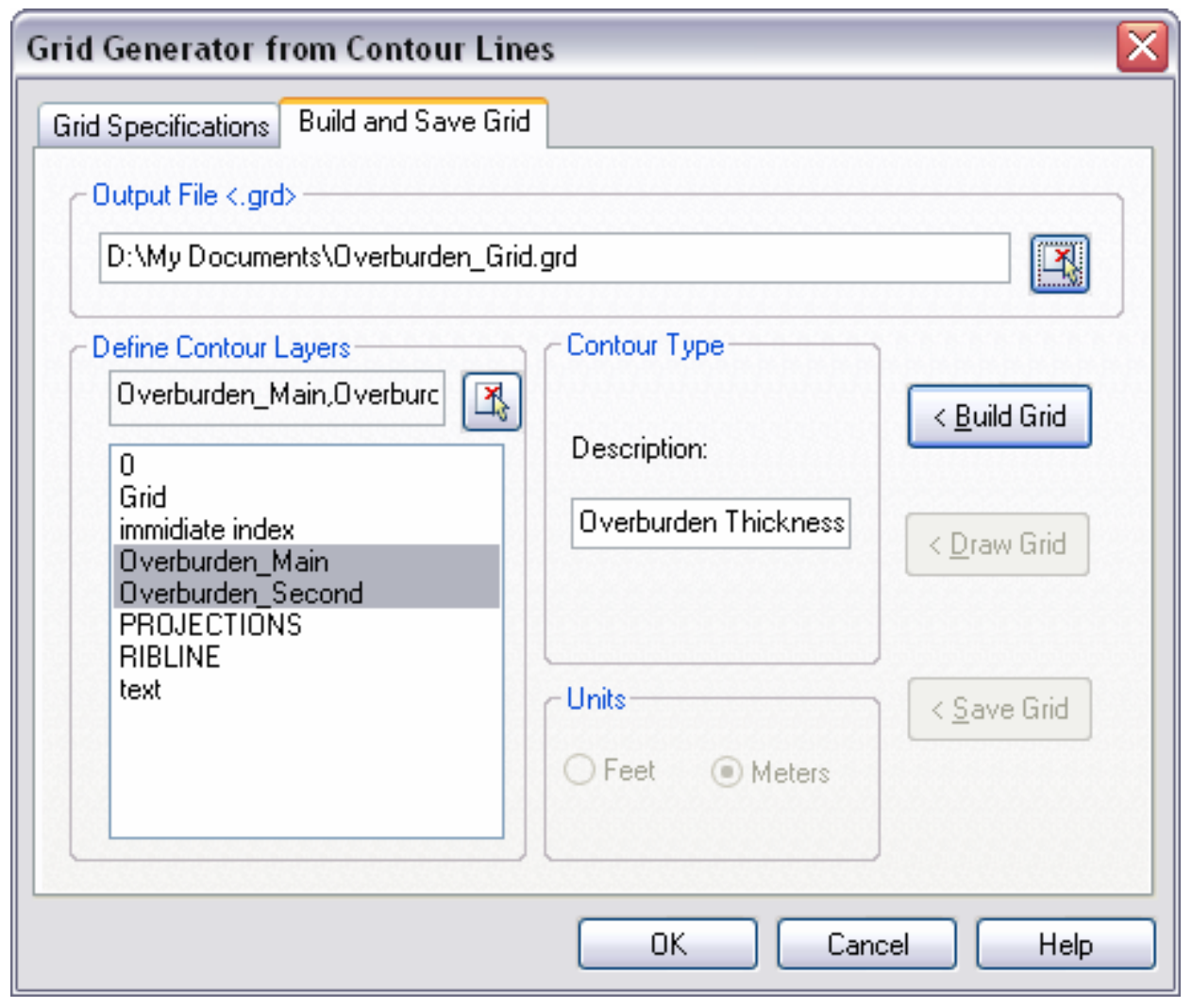

Figure B.4 Form of contour gridding module 
The main inputs for this module are the names of the layers where contours are, and the output grid file name. After the grid is built (command Build Grid) from specified contours, the results can be displayed (command Draw Grid) and stored into file (command Save Grid).

- Grid generation for survey point form

This is the input form for creating geologic influence factor grid for 3D point-type data (see Figure B.5). The input data can be the points in map layers or an external file. The Min. Points parameter specifies the minimum number of neighbor points used in the grid interpolation. The Max. Search $\boldsymbol{R}$ parameter is the maximum radius to search for the neighbor points. The interpolation methods implemented in this module are a Simple Average and an Inversed Distance method.

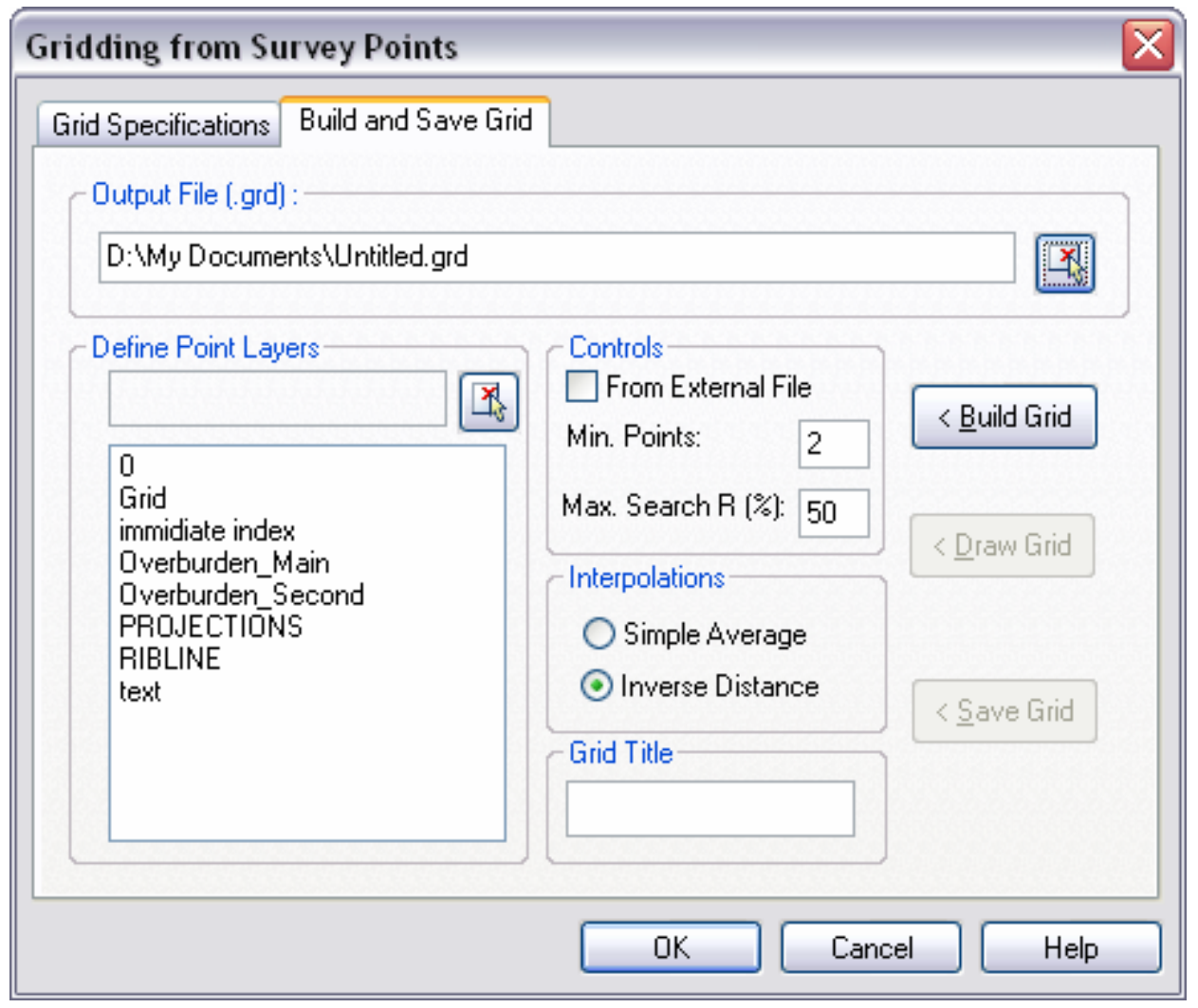

Figure B.5 Form for point gridding module 
- Grid generation for linear geological feature form

This form (see figure B.6) is provided to create a grid from linear geological features. In addition to the feature layers and the output grid file name, the Influence Distance of the features is required as main input to specify the range of the influence around the features. This parameter defines a rectangular box with a width of two times of the Distance and a length of the linear feature. If a grid element is inside the box, an influence value will be assigned to the grid element by an inverse linear interpolation. The optional Accumulation allows summing up the influences on the area affected by multiple features. It means when a grid element is impacted by multiple features, all influence values for these features will be calculated. If Accumulation is set, the final influence factor for this grid element will be the summation of theses individual values. Otherwise, the largest value will be set to that grid element.

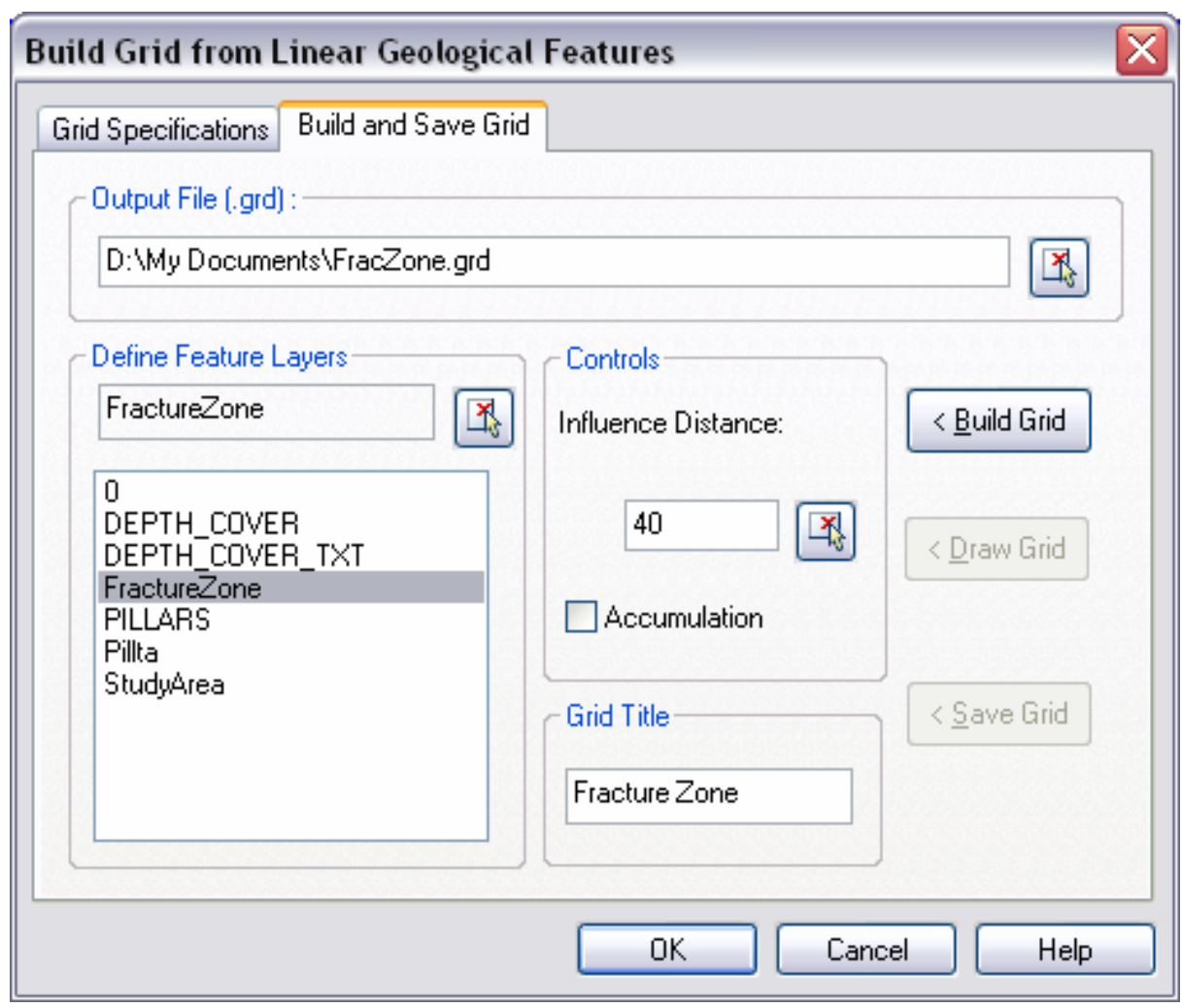

Figure B.6 Form for linear geological feature module 
- $\quad \underline{\text { Input form for area based geology features }}$

The form for gridding the area-based geological feature module includes two more tabs besides the standard grid definition sheet. The tab Boundary Creation (see Figure B.7) provides the abilities to interactively create and manage the influence boundaries for the geology structures. These influence boundaries can be created from linear objects in layers, individual center lines, and area boundaries. The Influence Range specifies the offset distance to the objects from which the boundaries are to be created.

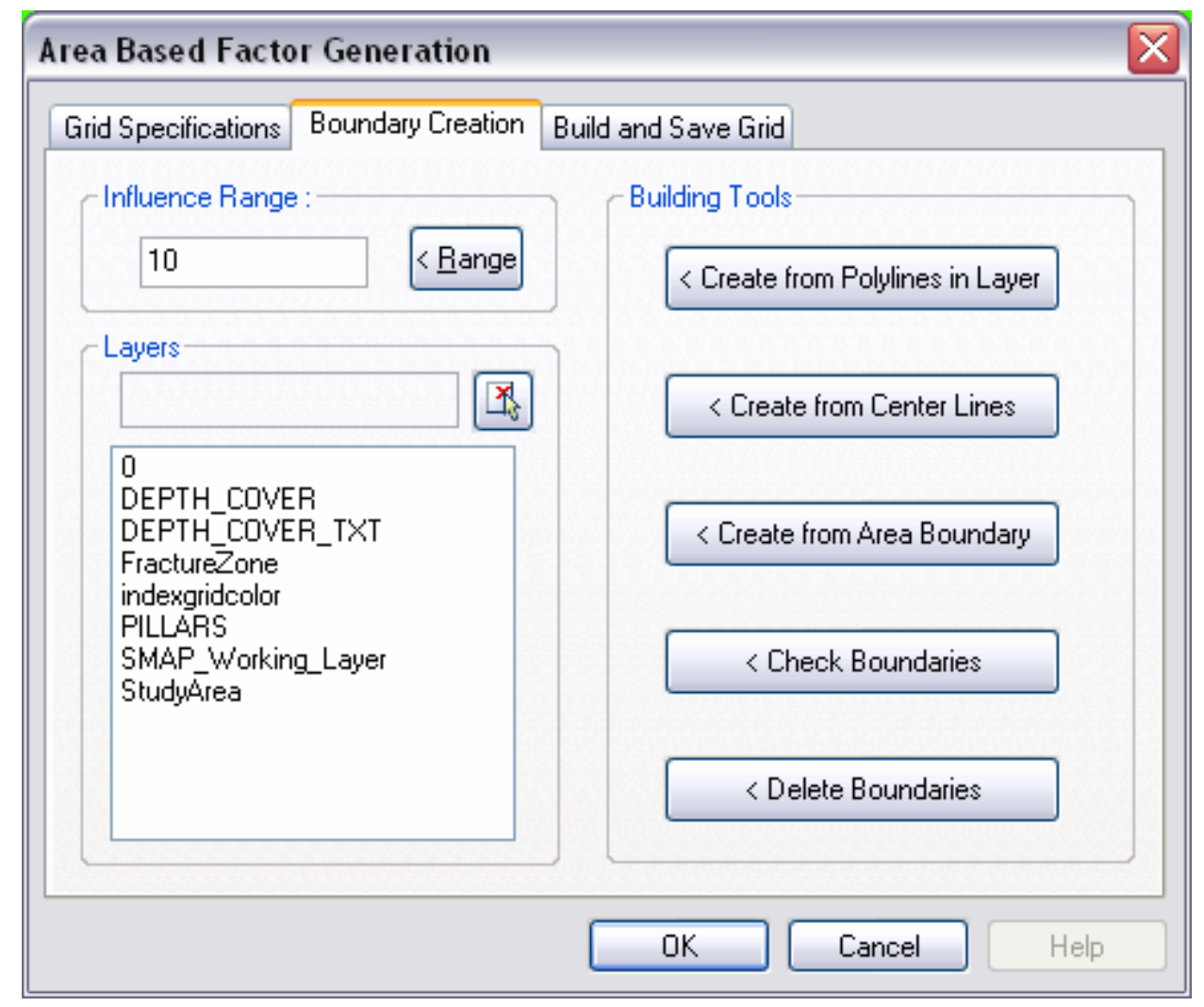

Figure B.7 Form for boundary creation 
The tab Build and Save Grid (see Figure B.8) is used to specify the output file name, grid values for inside/outside element, and algorithm of gridding. Currently, the Middle Point and the Area Based methods are implemented in this module (see Chapter 4.3.4). After the grid is built by using command Build Grid, the values of elements can be checked and changed interactively in AutoCAD model space (command Change Cells). If Remove Boundaries is checked, all boundaries created in the previous step will be erased from AutoCAD database after the grid is built.

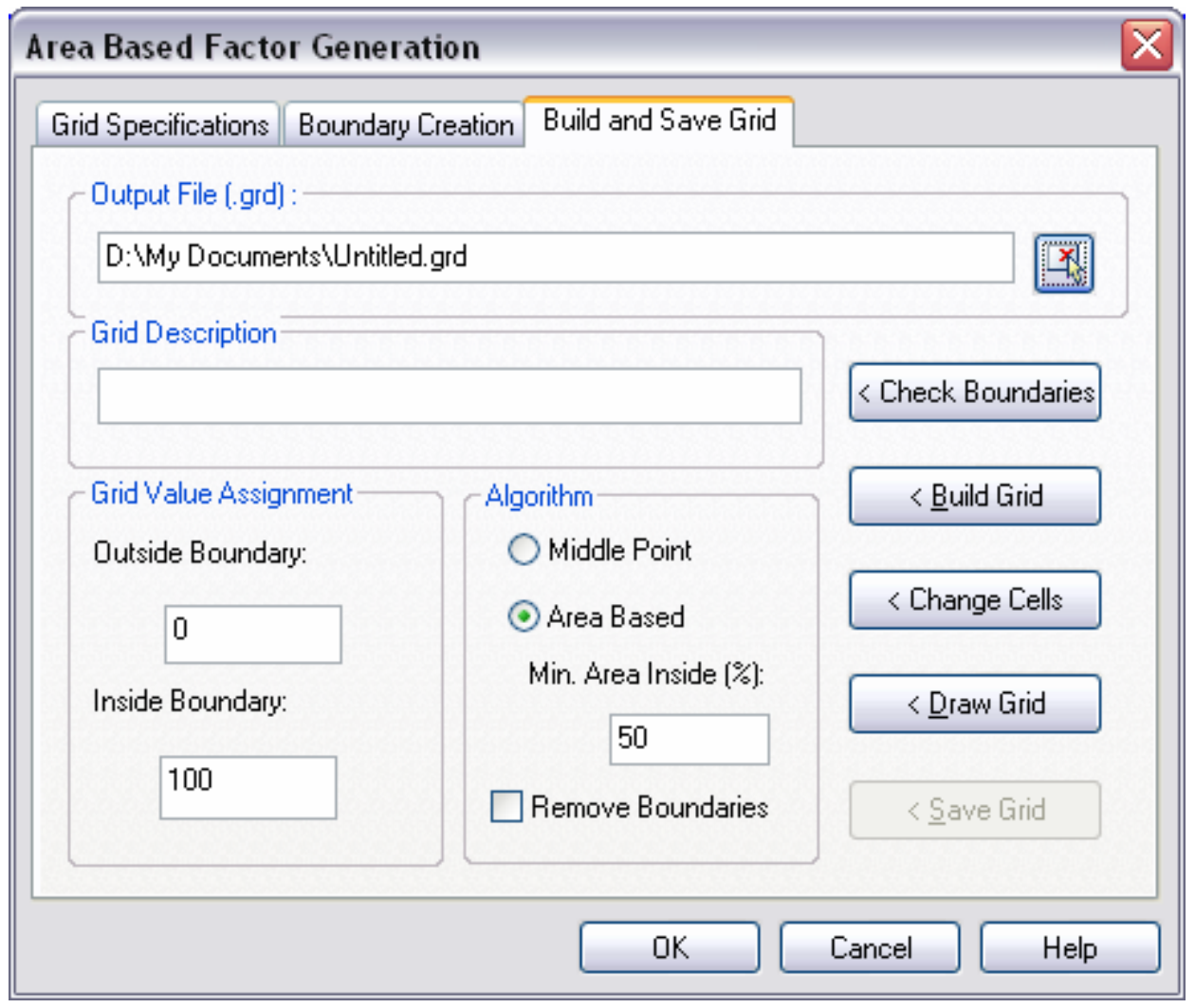

Figure B.8 Form for area based factors 
- Input forms for overburden stress module

The overburden stress calculation module calculates seam stress from an overburden grid. The required input parameters are grouped into three forms. The first form (Figure B.9) is used to specify the surface grid. This grid should be created previously by using the stability system geologic modules or SurvCADD mining modules. The value stored in the surface grid can be Overburden Thickness or Elevation of the topography.

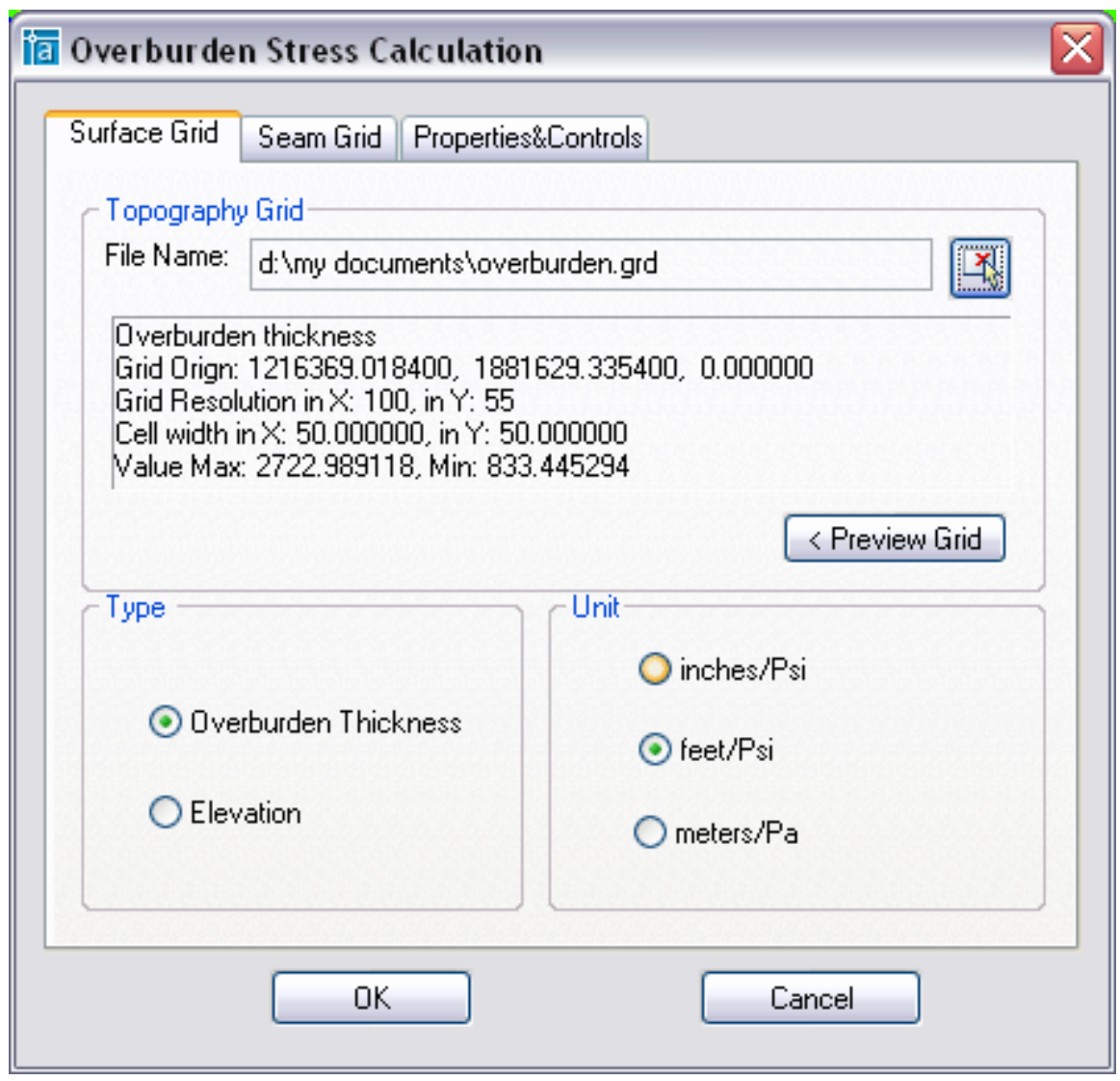

Figure B.9 Form for surface grid in overburden stress module 
The second form (Figure B.10) defines the seam grid specifications and the output file name. The seam grid definition can be newly defined (command New) or referenced to a existing grid file (command From File). If the topography grid contains the elevation of the surface, then the elevation of seam should be specified in this form. The Seam Level can be input as a single value or use the average value of the current seam grid.

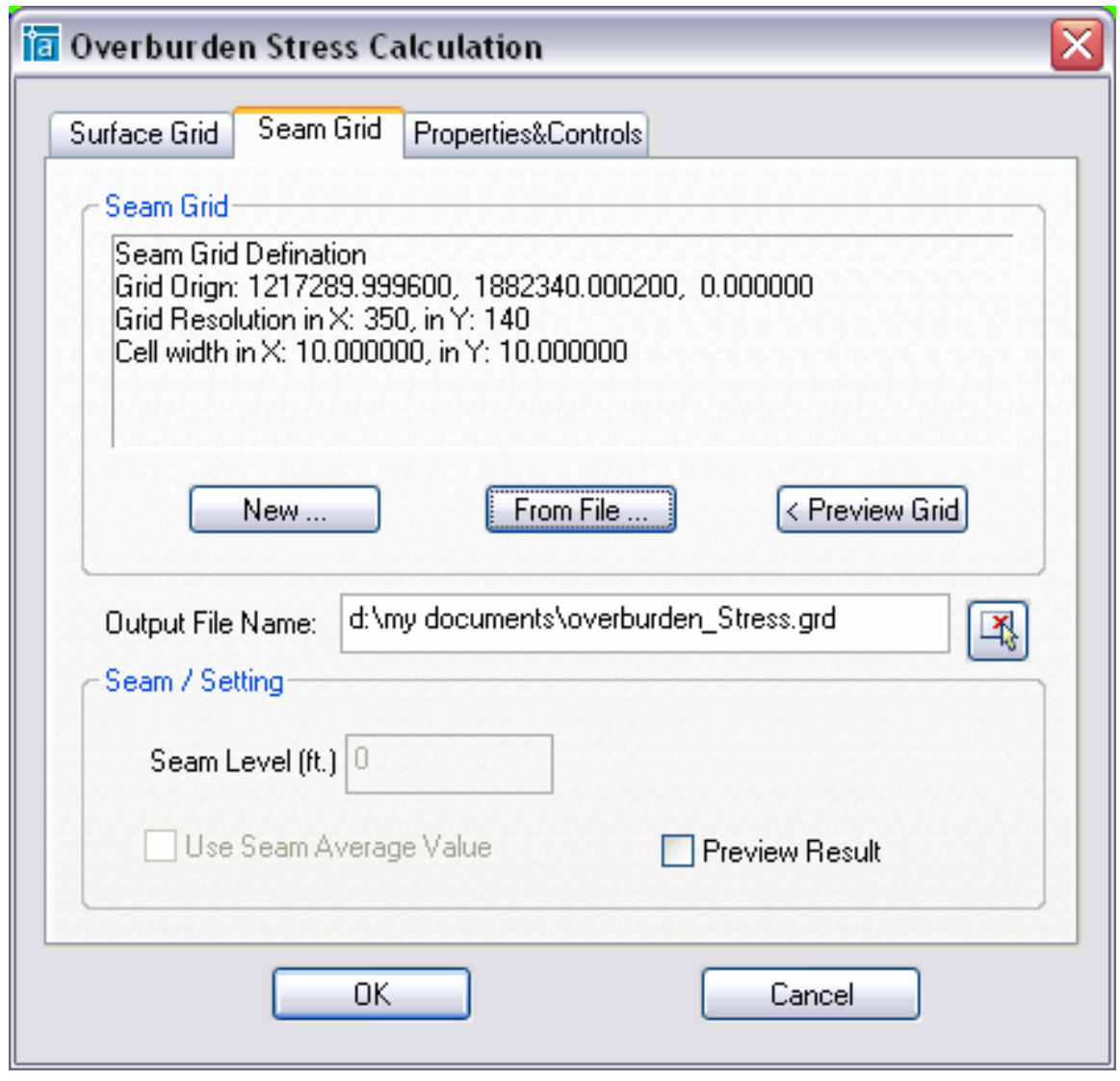

Figure B.10 Form for seam grid in overburden stress module 
The properties and controls parameters form (Figure B.11) allows the user to adjust the parameters for the stress projection from the overburden to the seam. This includes the laminated overburden physical properties such as the Lamination Thickness, the Elastic Modulus, the Vertical Stress Gradient and the Poisson's Ratio, and the control parameters namely the Maximum Number of Iteration, the Displacement Convergence Level and the Over Relax Factor. Detailed explanation of these parameters can be seen in Chapter 4.4 and references (Heasley, 1998)

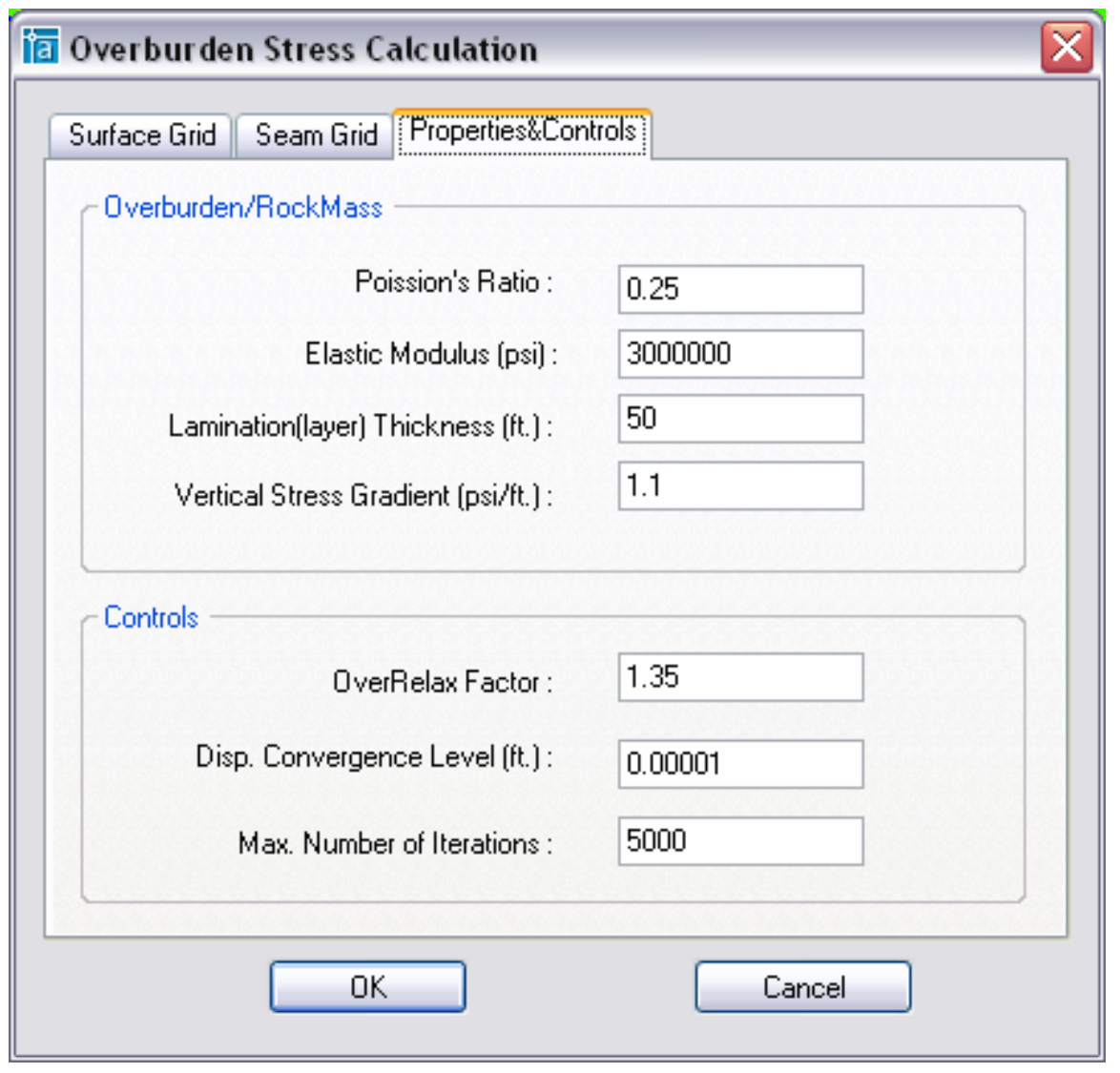

Figure B.11 Form for properties and control parameters 
- Input form for horizontal stress impact

This is the input form for horizontal stress module. In addition to the output grid file name and the layer names of entry projections, the Width of Entries and the Orientation of Max Stress are required inputs (see figure B.10). The orientation of max stress is the orientation of the maximum horizontal stress. These two parameters can be either typed in the text box or specified from AutoCAD model view interactively.

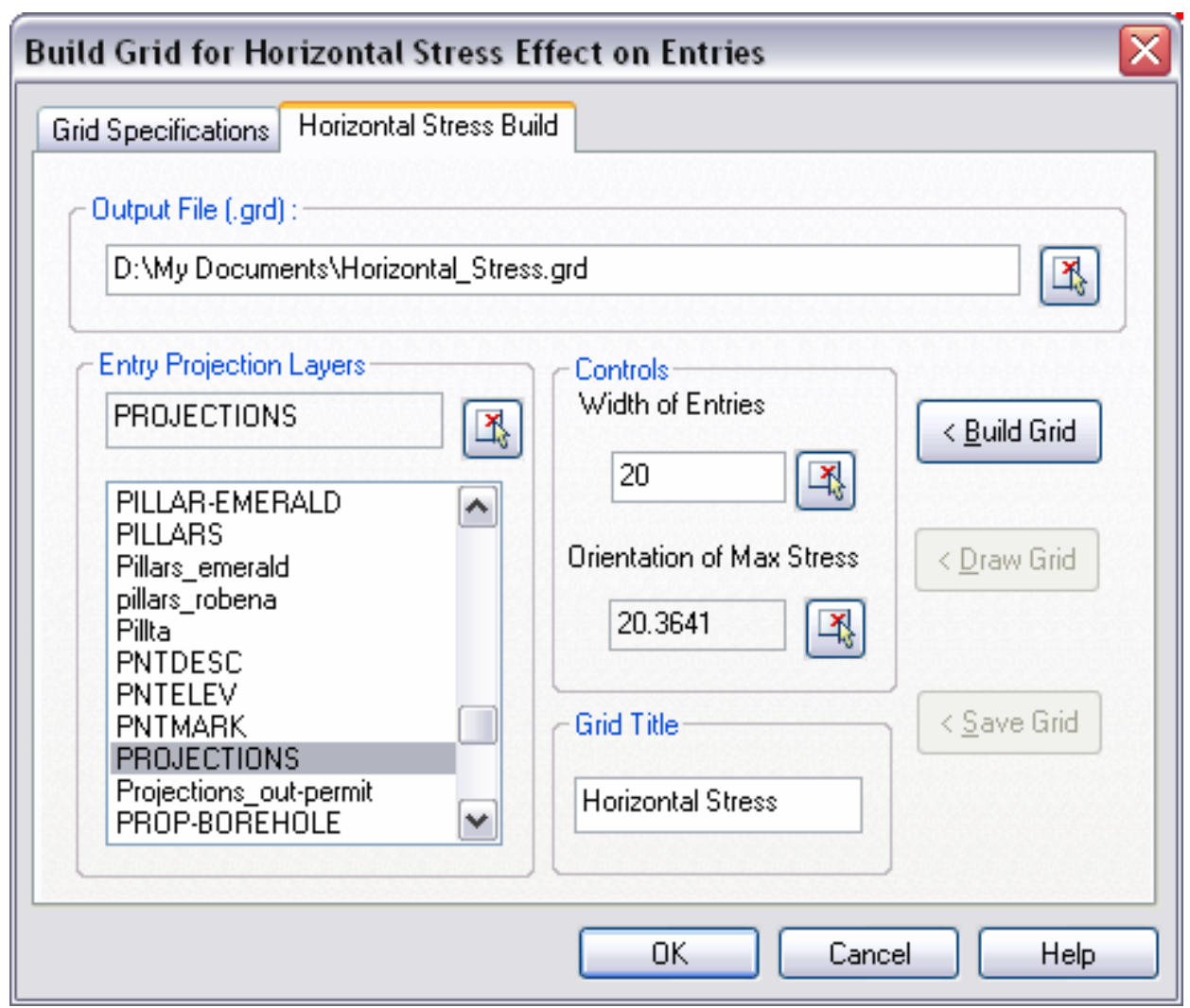

Figure B.12 Form for horizontal stress influence 
- Influence factor transformation form

These two forms (see Figures B.13, B.14) provide a wizard type interface to transform the original values of the stability influence factors into individual stability indices. The first form (see Figure B.13) is used to specify the original factor grid file name to be transformed and the output grid file name where the resultant individual index will be saved. Optionally a description of the index can be inputted.

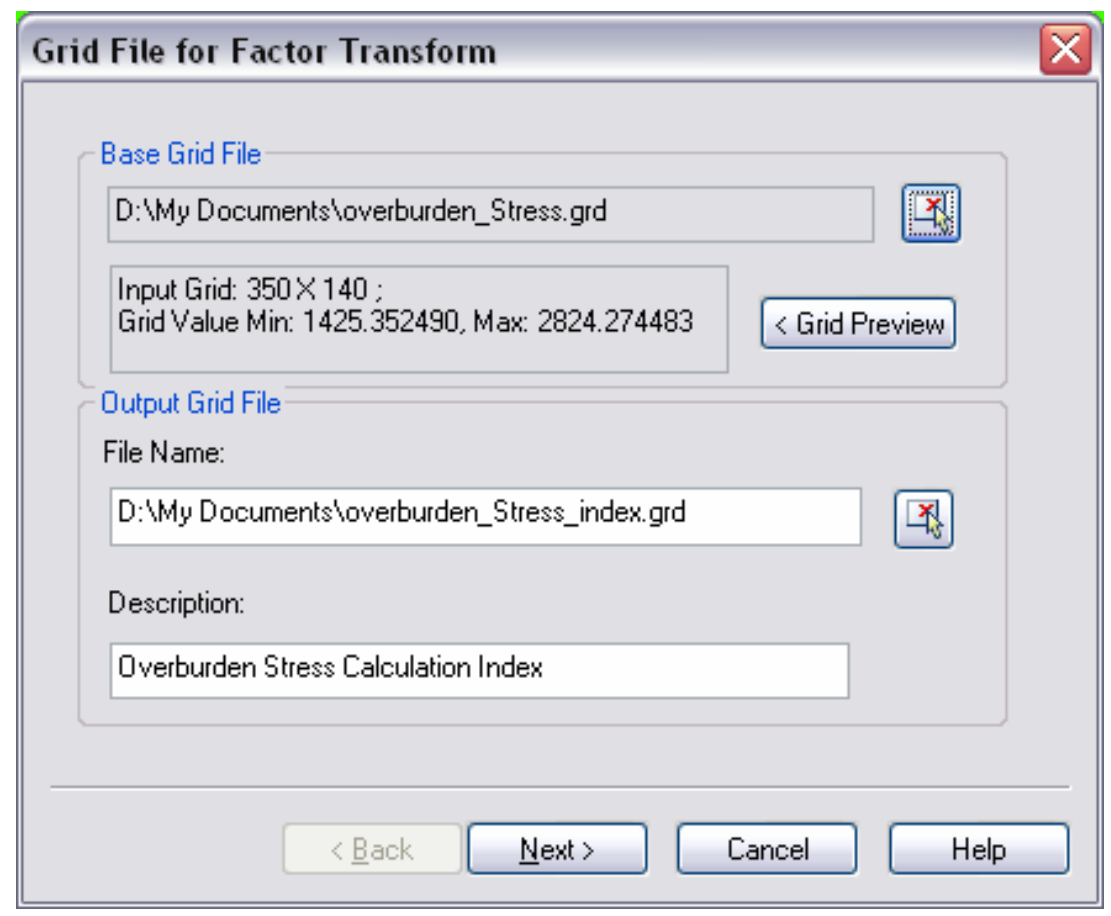

Figure B.13 Form for original grid in transformation module 
The second form (see Figure B.14) provides the specification of the transformation method. Currently available methods are Linear, Step and Exponential. The option Increasing tells the module to perform a normal transformation, while Decreasing will cause an inversed transformation. The Lower and Upper Bounds define the value range over which the transformation method will occur. The Finish command will start the transformation process and write the results to the predefined output grid file.

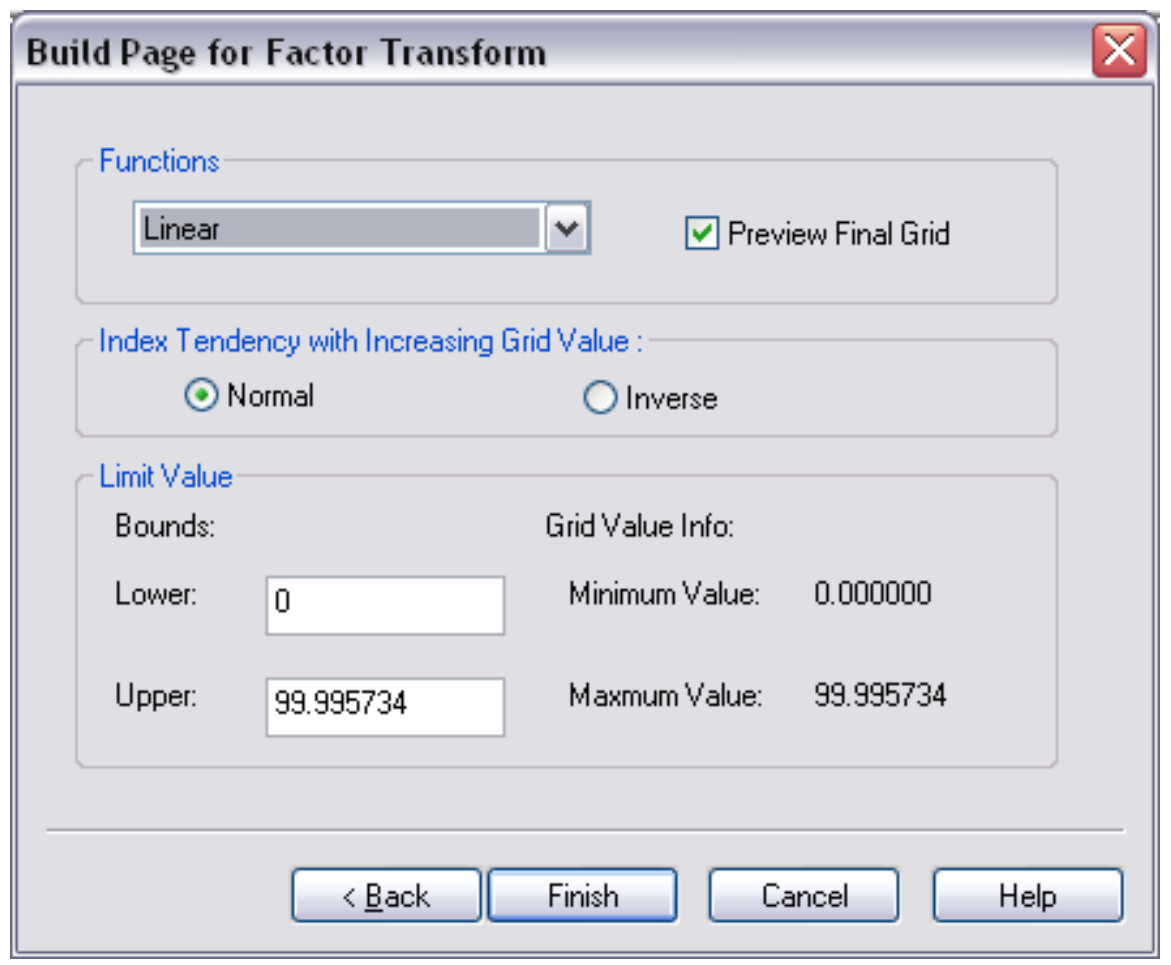

Figure B.14 Form for influence factor transformation 
- Stability index generation form

This form provides the interface for calculating the final overall stability index from the multiple influence factors by using a weighted average method (see Figure B.15). For convenience, a project file with extension .prj can be defined for each calculation. The project file can be saved and read any time. This allows conveniently trying different weights and combinations of the influence factors by saving current setting into a project file.

The command Select Index Grid is used to selected the grid files of the individual stability indices. The weighting factor for the individual index should be given between 1 and 100 with the higher weights associate with the more important factors. The individual index can be added to, removed from, or updated in the list box. The command Create Index calculates the stability index using the current influence factors and weights in the list. The resultant stability index would be saved to a specified grid file.

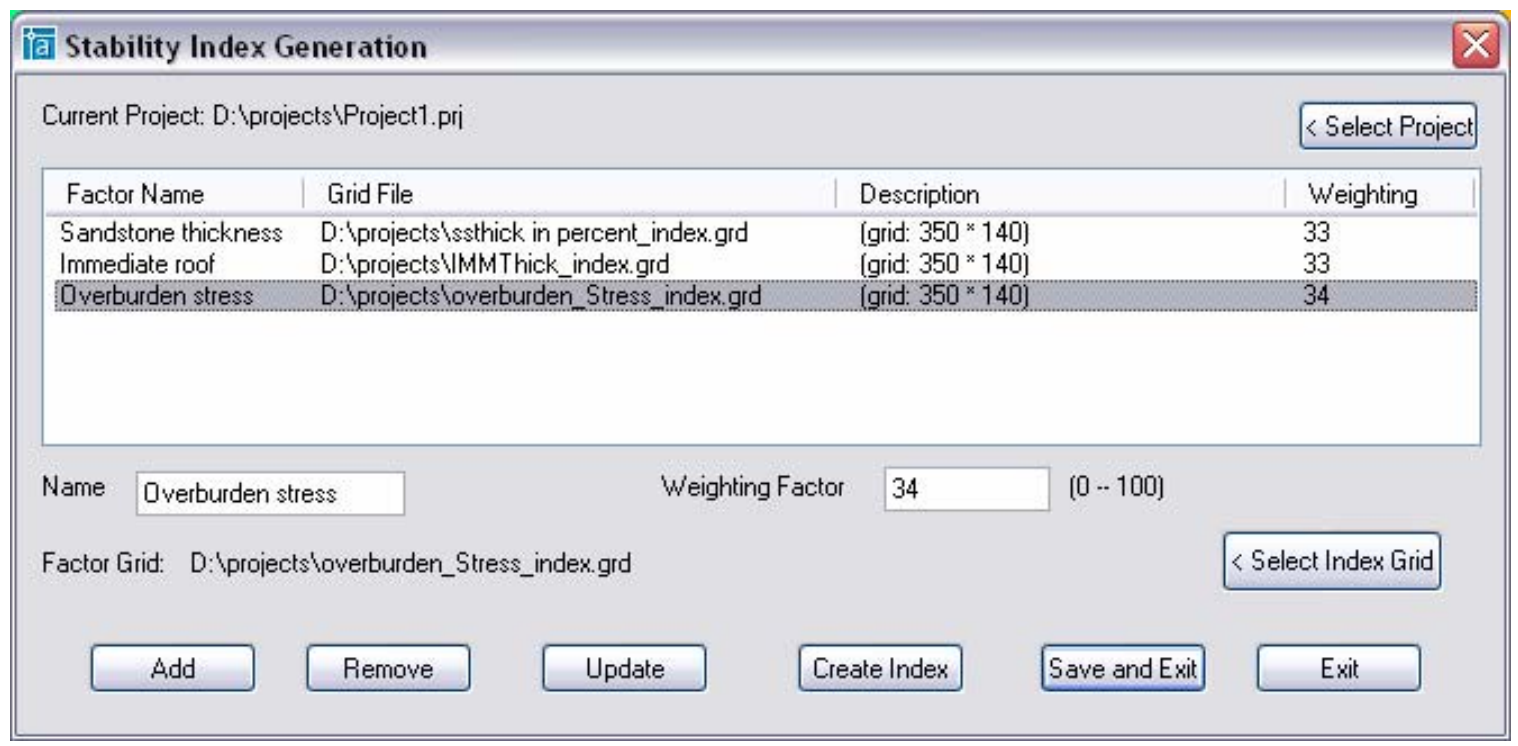

Figure B.15 Form for stability index generation 
- $\quad$ Stability Index Mapping Form

Stability index mapping is a utility function for mapping the stability index to the mine entries. The resultant drawing can be used to guide the mine engineers in ground control and support design, or to make a comparative study with field observations (see case studies in Chapter 6).

To create the map, the Pillar Layers where the pillar polylines resided, and the Mapping Layer where the index will be drawn need to be specified in the form (see Figure B.16). Optionally, the index value range to be plotted in the grid can be defined. The command Analyze starts the process to calculate and construct the regions of grid element overlaying on the entries. The command Pick Region can be used to select existing regions previously constructed in the mine map. The command Hatch will fill the regions with the various colors based on the related index values.

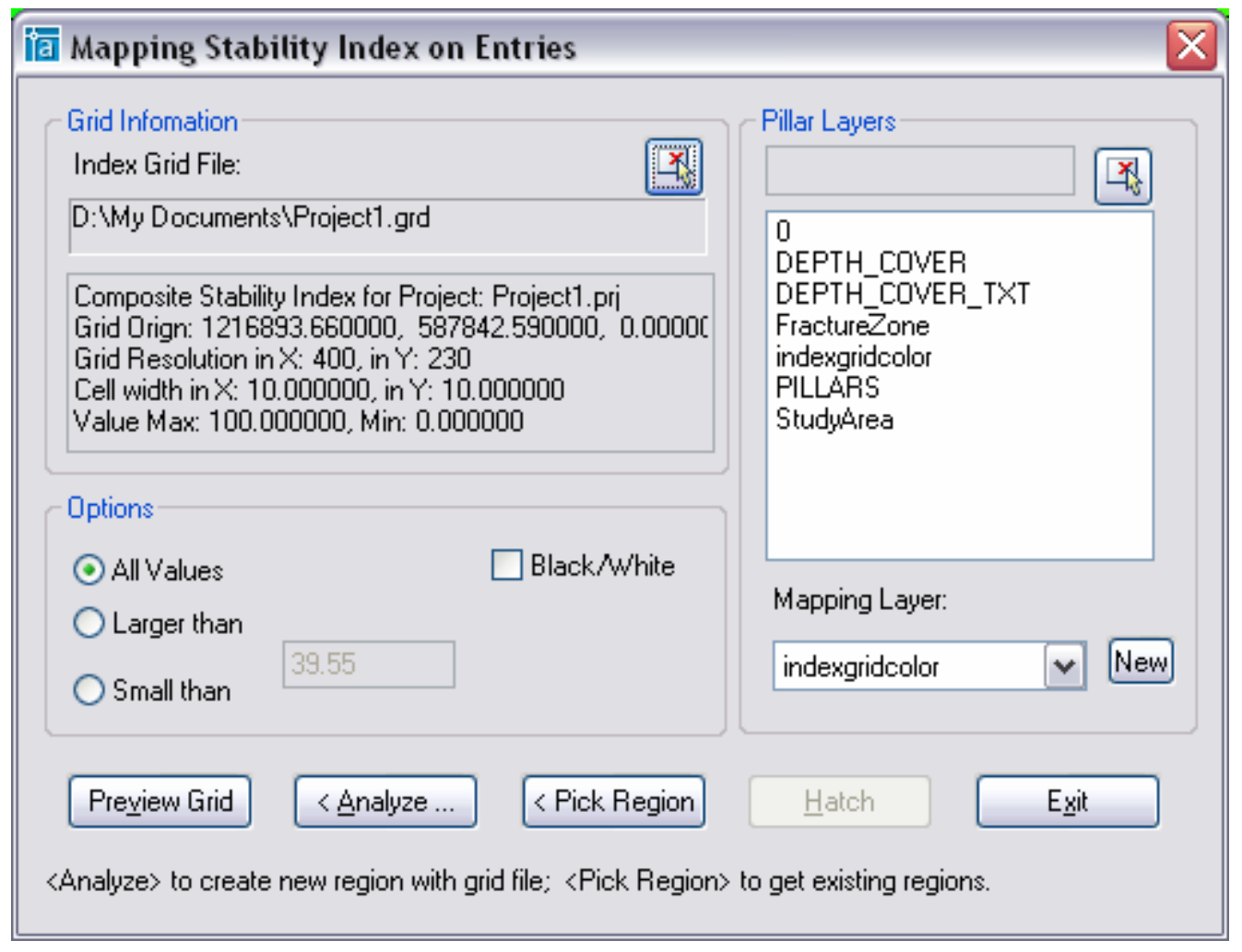

Figure B.16 Form for stability index mapping 


\section{- $\quad$ Grid utility form}

This form (see Figure B.17) provides accesses to the comprehensive grid utility function implemented in the stability mapping system. The functions included in this form are the grid file management, grid value management and grid plotting.

Grid file management includes the functions to create New Grid, Read value from other grid file, and to Save grid to file. Grid value management functions can Move grid location, change individual element value, apply basic arithmetic operations to a grid or between two grids. Plotting contains a number of useful tools to can be used to Preview and Plot a grid with various drawing settings.

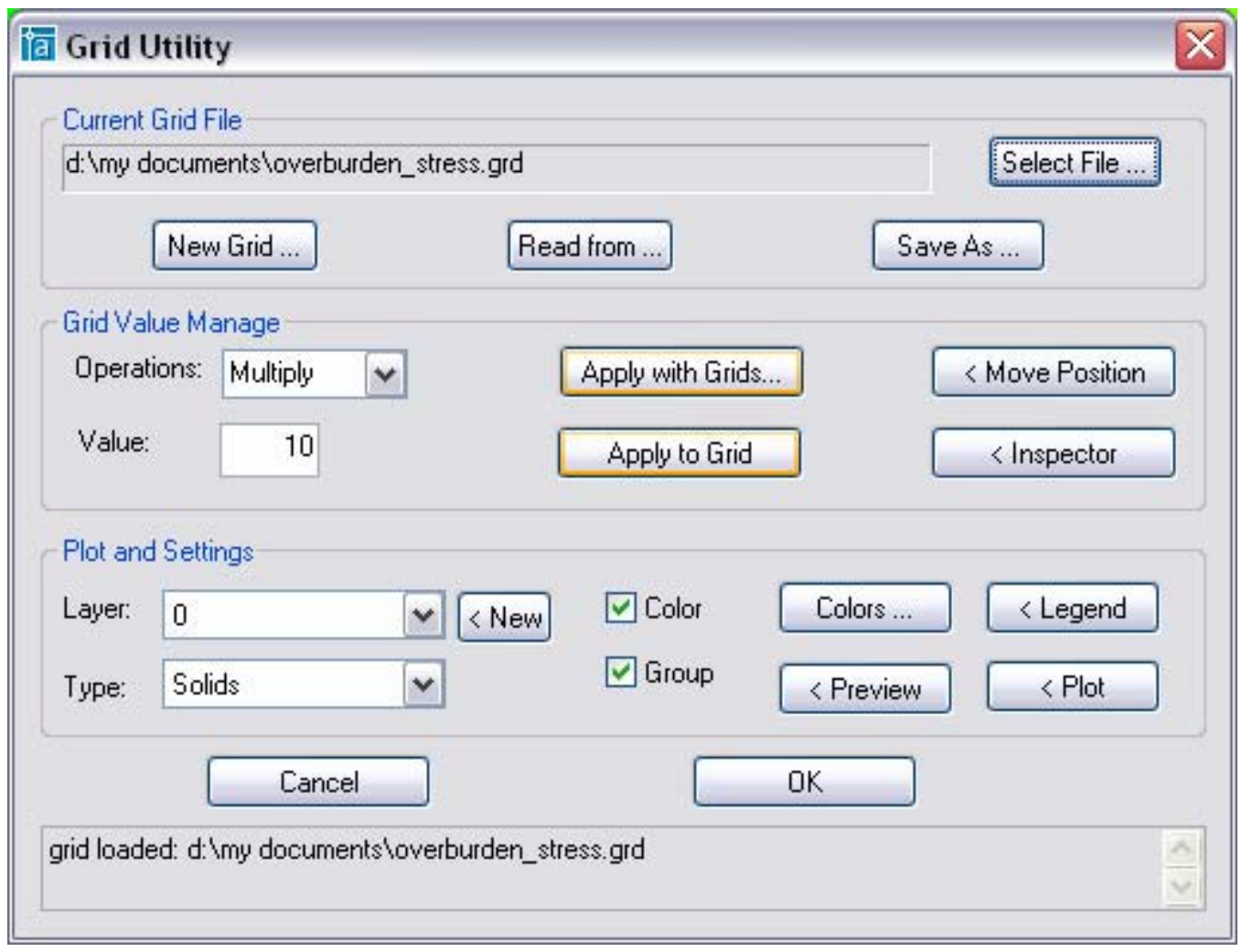

Figure B.17 Grid utility form 


\section{Appendix C. AutoCAD Database Overview}

An AutoCAD drawing is a collection of objects stored in a database. Some of the basic database objects are entities, symbol tables, and dictionaries. Entities are a special kind of database object that have a graphical representation within an AutoCAD drawing. Lines, circles, arcs, text, solids, regions, splines, and ellipses are examples of entities. A user can see an entity on the screen and can manipulate it.

Symbol tables and dictionaries are containers used to store database objects. Both container objects map a symbol name (a text string) to a database object. An AutoCAD database includes a fixed set of symbol tables, each of which contains instances of a particular class of symbol table record. You cannot add a new symbol table to the database. Examples of symbol tables are the layer table (AcDbLayerTable), which contains layer table records, and the block table (AcDbBlockTable), which contains block table records. All AutoCAD entities are owned by block table records.

Dictionaries provide a more generic container for storing objects than symbol tables. A dictionary can contain any object of the type AcDbObject or subclass thereof. The AutoCAD database creates a dictionary called the named object dictionary when it creates a new drawing. The named object dictionary can be viewed as the master "table of contents" for all of the dictionaries associated with the database. You can create new dictionaries within the named object dictionary and add new database objects to them.

The following figure C-1 shows the key components of the AutoCAD database. 


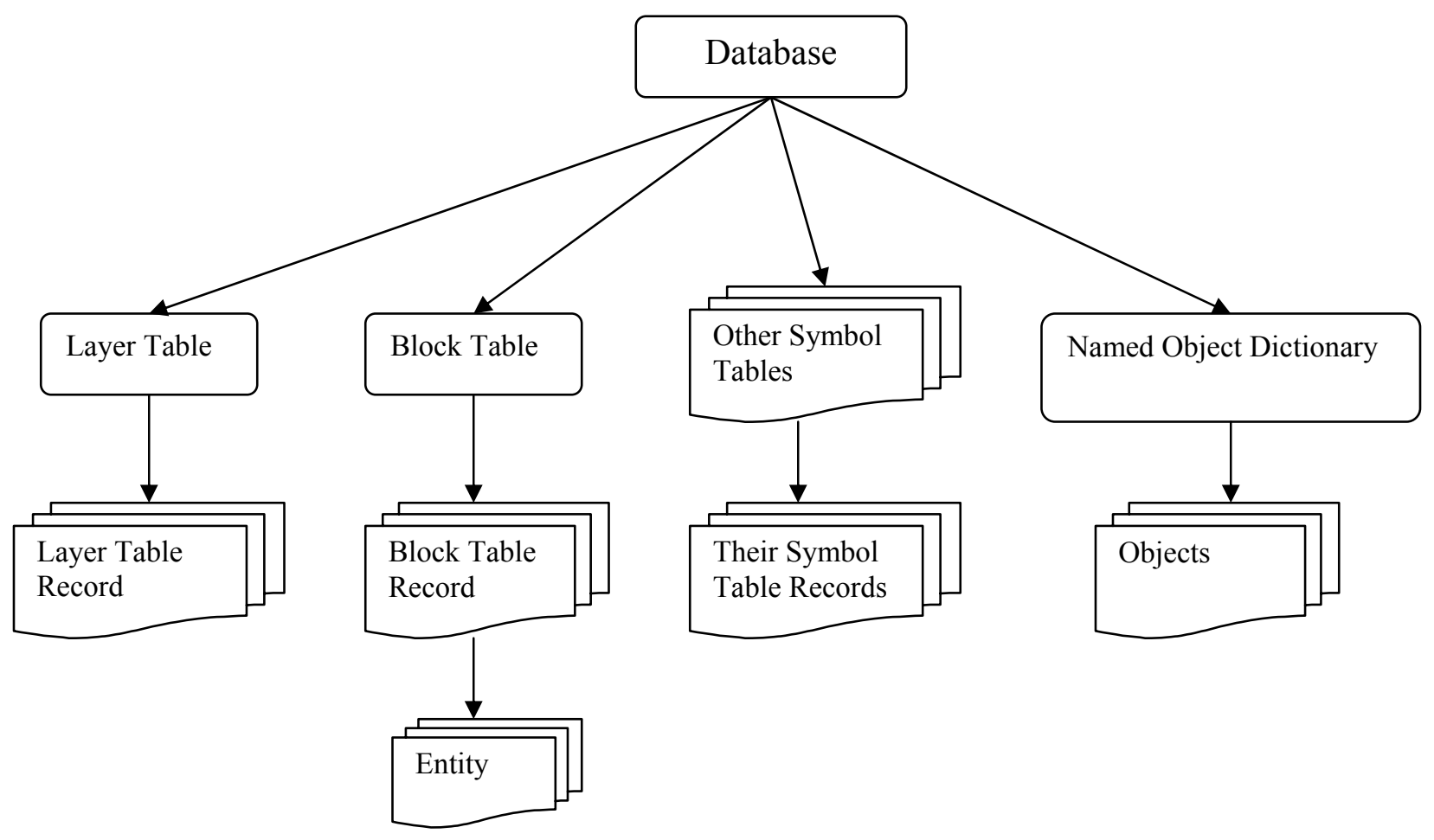

Figure C-1 AutoCAD Database Components

In AutoCAD mining maps, the most used linear graphic representation is the Polyline, a connected multi-segment-line. These polylines are grouped into different layers according to their physical meanings. For examples, all pillar outlines may be in a layer named "Pillar". This property provides a convenience for the stability application to automatically access the massive data input by searching through the specified layers. 


\section{VITA}

QUANXI WANG was born in Yuci city, Shanxi province, People's Republic of China. He spent his childhood in the suburb of the city before he left for college in 1982. He received his Bachelor of Science Degree in Mining Engineering from Taiyuan University of Technology, Taiyuan, China in 1986. In April 1989, he earned a Master of Science Degree in Mining Engineering from Shandong University of Science and Technology, Taian, China.

From 1989 to 2001, Mr. Wang had worked as a mining engineer with Taiyuan Coal Mine Design and Research Institute, one of the top mine design and consulting organizations in China. He had involved a number of mine design projects, and also was responsible for computer application, CAD application and mining software development.

From spring, 2002, Mr. Wang started his Ph. D. program in Department of Mining Engineering, West Virginia University. Currently he is a doctoral candidate and a student member of Society of Mining, Metallurgy and Exploration (SME). 